University of Tennessee Health Science Center

UTHSC Digital Commons

\title{
$5-2018$
}

\section{Health-Related Quality of Life in Female Children with Congenital Adrenal Hyperplasia: A Mixed Methods Study}

Lacretia Carroll

University of Tennessee Health Science Center

Follow this and additional works at: https://dc.uthsc.edu/dissertations

Part of the Pediatric Nursing Commons

\section{Recommended Citation}

Carroll, Lacretia (http://orcid.org/0000-0002-3989-1352), "Health-Related Quality of Life in Female Children with Congenital Adrenal Hyperplasia: A Mixed Methods Study" (2018). Theses and Dissertations (ETD). Paper 459. http://dx.doi.org/10.21007/etd.cghs.2018.0452. 


\title{
Health-Related Quality of Life in Female Children with Congenital Adrenal Hyperplasia: A Mixed Methods Study
}

\begin{abstract}
Introduction. Health-related quality of life (HRQOL) has become a meaningful area in research, medical management, and in the evaluation of health outcomes. However, in children with congenital adrenal hyperplasia (CAH), HRQOL is a long-neglected subject. Previous research in individuals with CAH typically focused on medical treatment and outcomes, which suggested that HRQOL is influenced by personal factors such as genital ambiguity, impaired growth, higher incidence of obesity, metabolic syndrome, hypertension, acute adrenal crisis, as well as losses in psychosexual milestones and psychological functioning. Social contexts, such as family, were identified as factors that contribute to an individual's perceived HRQOL. Today, there is some agreement that non-disclosure, shame, secrecy, and stigma affect psychological well-being. Despite research efforts, there is limited information about which factors influence HRQOL and which factors are most relevant to female children with $\mathrm{CAH}$. This indicates the need to go beyond identifying single factors, and address the complex relationship of factors that impact HRQOL, and individuals' perception of these factors. Therefore, the bioecological model of human development was used to understand the different factors that impact HRQOL in female children with $\mathrm{CAH}$.

Methods. A convergent mixed methods design was selected whereby the quantitative and qualitative results were merged to provide a comprehensive understanding of HRQOL of female children with CAH. Qualitative methods (QUAL) involved using semi-structured interviews with child-caregiver dyads $(\mathrm{N}=20)$ to explore $\mathrm{HRQOL}$ and describe factors that impact $\mathrm{HRQOL}$ of female children with $\mathrm{CAH}$. Member checking procedures were implemented to ensure trustworthiness or credibility and rigor of the QUAL component of this study. NVivo 10 was used to analyze the QUAL data. Additionally, the full sample of 25 child-caregiver dyads completed KINDL-R questionnaires, which provided a quantitative (QUAN) measure of HRQOL of female children with CAH. QUAN data were analyzed using SPSS version 24. Individual analysis of both QUAL and QUAN data were merged to ascertain findings for a mixed methods interpretation.
\end{abstract}

Results. Children with $\mathrm{CAH}$ and their caregivers reported having good overall HRQOL. Children scored significantly lower on the School subscale compared to their caregivers $(M d n=12.5, I Q R=18.75)$ Examination of the data revealed significant relationships between other health issues and how caregivers rated children's HRQOL, $p<.05$. The following themes emerged from the child and caregiver interviews: 1) living with $\mathrm{CAH}$; 2) normalcy; 3) disclosure of diagnosis; 4) feelings of the child/caregiver; 5) what should we know; and 6) improvements. Merging of data showed that not all of QUAN variables are reflected in the QUAL themes. However, many of the QUAL categories and comments elucidated information about the spectrum of children's and caregiver's perceptions of the HRQOL of children with $\mathrm{CAH}$.

Discussion. Using a comprehensive approach offered an understanding of what it is like for children to live with $\mathrm{CAH}$ from the perspective of the child and caregiver. By quantitatively measuring HRQOL and using qualitative interview data, the information in this study may shed light on children's and caregivers' needs that are not met by current guidelines. Although the overall child- and caregiver-reported HRQOL of female children was not impaired, children's and caregiver's lived experiences highlight the many factors that impact HRQOL including the importance of friends and family, personal characteristics (i.e., the ability to adapt and self-esteem), the environment (i.e., home, school), and the impact of time (i.e., developmental time, past vs present). Knowing this, clinicians may better support children and families by expanding the focus beyond sex development to include: 1) information about the different ways of knowing (i.e., knowing how to talk with others and with their child); 2) self-management and 
independence (i.e., learning how to administer medication and handling responsibility as children grown older); and 3) psychological support (i.e., coping with a new diagnosis, adapting to the disorder.)

\section{Document Type}

Dissertation

Degree Name

Doctor of Philosophy $(\mathrm{PhD})$

\section{Program}

Nursing Science

\section{Research Advisor}

Carolyn Graff, Ph.D.

\section{Keywords}

congenital adrenal hyperplasia, disorders of sexual development, female children, health-related quality of life, mixed methods

\section{Subject Categories}

Medicine and Health Sciences | Nursing | Pediatric Nursing 
Health-Related Quality of Life in Female Children with Congenital Adrenal Hyperplasia: A Mixed Methods Study

\author{
A Dissertation \\ Presented for \\ The Graduate Studies Council \\ The University of Tennessee \\ Health Science Center \\ In Partial Fulfillment \\ Of the Requirements for the Degree \\ Doctor of Philosophy \\ From the University of Tennessee
}

By

Lacretia Carroll

May 2018 
Copyright $(\mathcal{}) 2018$ by Lacretia Carroll. All rights reserved. 


\section{DEDICATION}

This work is dedicated to everyone who supported me during one of the most challenging paths I have ever traveled. To my God parents, mom, and grandparents thank you for always supporting me throughout this entire journey. JB Smiley Jr., thank you for encouraging me, and pushing me to the finish line. To Dr. April Epps-Carter, thank you for being one of my biggest supporters and cheerleaders throughout every stage of our nursing careers, especially this one. Your encouraging words and consistent prayers do not go unnoticed. To all of my immediate and extended family, thank you for your prayers, patience, and understanding. I also want to dedicate this work to each caregiver and child that welcomed me into the most intimate parts of your life. It is my prayer that this work sheds light to the healthcare community, and benefits one child in some way. 


\section{ACKNOWLEDGEMENTS}

To Dr. Graff, my dissertation committee chair, thank you for sharing qualitative and mixed methods expertise, and challenging me to think differently. I also want to thank you for your guidance, patience, for believing in me, and continued support throughout this program.

I would also like to thank the other members of my committee: Dr. Mona Wicks who provided me with invaluable words of encouragement who made me want to continue with the work, persevere, and who helped me believe that my work is beneficial to the literature on children with congenital adrenal hyperplasia $(\mathrm{CAH})$; Dr. Donna Hathaway who shared her experience with $\mathrm{CAH}$ and expertise on quality of life as well as the significance of research; Dr. Alicia Diaz Thomas who shared her expertise on $\mathrm{CAH}$, provided me with resources, and introduced new and innovative ways to immerse myself in this population, Dr. Satish Kedia who shared his expertise on methodology and who pushed me to write high quality, publishable research, and Dr. Tamekia Jones who patiently guided me through the intimidating process of statistical analysis.

Lastly, I want to show my appreciation to the Cares Foundation for assisting me with patient recruitment, and offering financial support to support this dissertation research. I would also like to thank Dr. Oksana Lekarev at the Comprehensive Center for Congenital Adrenal Hyperplasia, New York- Presbyterian Komansky Children's Hospital, New York, New York for allowing me to learn and immerse myself in her patient population. Lastly, I would like to thank the Beta Theta-at-Large Chapter of Sigma Theta Tau International Honor Society of Nursing and the University of Tennessee Health Science Center College of Nursing for awarding me a scholarship and grant to cover the cost of gift cards, expenses related to travel, and administrative costs related to this dissertation research. 


\begin{abstract}
Introduction. Health-related quality of life (HRQOL) has become a meaningful area in research, medical management, and in the evaluation of health outcomes. However, in children with congenital adrenal hyperplasia (CAH), HRQOL is a long-neglected subject. Previous research in individuals with CAH typically focused on medical treatment and outcomes, which suggested that HRQOL is influenced by personal factors such as genital ambiguity, impaired growth, higher incidence of obesity, metabolic syndrome, hypertension, acute adrenal crisis, as well as losses in psychosexual milestones and psychological functioning. Social contexts, such as family, were identified as factors that contribute to an individual's perceived HRQOL. Today, there is some agreement that non-disclosure, shame, secrecy, and stigma affect psychological well-being. Despite research efforts, there is limited information about which factors influence HRQOL and which factors are most relevant to female children with CAH. This indicates the need to go beyond identifying single factors, and address the complex relationship of factors that impact HRQOL, and individuals' perception of these factors. Therefore, the bioecological model of human development was used to understand the different factors that impact HRQOL in female children with $\mathrm{CAH}$.
\end{abstract}

Methods. A convergent mixed methods design was selected whereby the quantitative and qualitative results were merged to provide a comprehensive understanding of HRQOL of female children with CAH. Qualitative methods (QUAL) involved using semi-structured interviews with child-caregiver dyads $(\mathrm{N}=20)$ to explore HRQOL and describe factors that impact HRQOL of female children with CAH. Member checking procedures were implemented to ensure trustworthiness or credibility and rigor of the QUAL component of this study. NVivo 10 was used to analyze the QUAL data. Additionally, the full sample of 25 child-caregiver dyads completed KINDL-R questionnaires, which provided a quantitative (QUAN) measure of HRQOL of female children with CAH. QUAN data were analyzed using SPSS version 24. Individual analysis of both QUAL and QUAN data were merged to ascertain findings for a mixed methods interpretation.

Results. Children with CAH and their caregivers reported having good overall HRQOL. Children scored significantly lower on the School subscale compared to their caregivers $(M d n=12.5, \mathrm{IQR}=18.75)$ Examination of the data revealed significant relationships between other health issues and how caregivers rated children's HRQOL, $p<.05$. The following themes emerged from the child and caregiver interviews: 1) living with $\mathrm{CAH}$; 2) normalcy; 3) disclosure of diagnosis; 4) feelings of the child/caregiver; 5) what should we know; and 6) improvements. Merging of data showed that not all of QUAN variables are reflected in the QUAL themes. However, many of the QUAL categories and comments elucidated information about the spectrum of children's and caregiver's perceptions of the HRQOL of children with CAH.

Discussion. Using a comprehensive approach offered an understanding of what it is like for children to live with $\mathrm{CAH}$ from the perspective of the child and caregiver. By quantitatively measuring HRQOL and using qualitative interview data, the information in 
this study may shed light on children's and caregivers' needs that are not met by current guidelines. Although the overall child- and caregiver-reported HRQOL of female children was not impaired, children's and caregiver's lived experiences highlight the many factors that impact HRQOL including the importance of friends and family, personal characteristics (i.e., the ability to adapt and self-esteem), the environment (i.e., home, school), and the impact of time (i.e., developmental time, past vs present).

Knowing this, clinicians may better support children and families by expanding the focus beyond sex development to include: 1) information about the different ways of knowing (i.e., knowing how to talk with others and with their child); 2) self-management and independence (i.e., learning how to administer medication and handling responsibility as children grown older); and 3) psychological support (i.e., coping with a new diagnosis, adapting to the disorder.) 


\section{TABLE OF CONTENTS}

CHAPTER 1. INTRODUCTION ......................................................................................

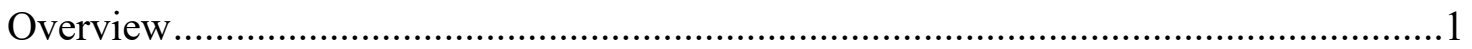

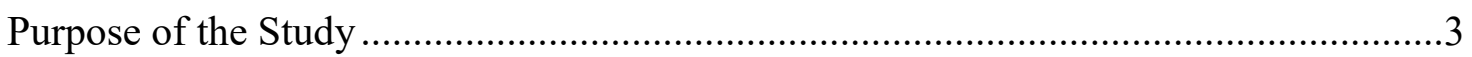

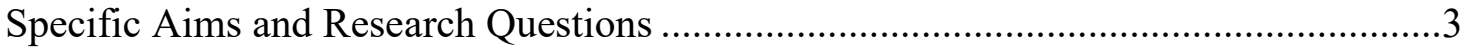

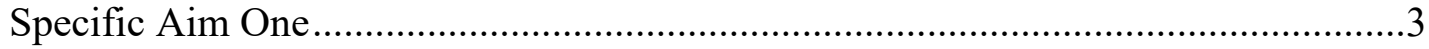

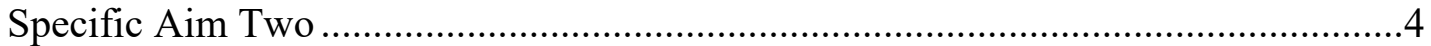

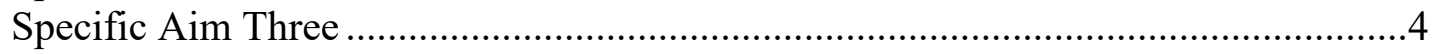

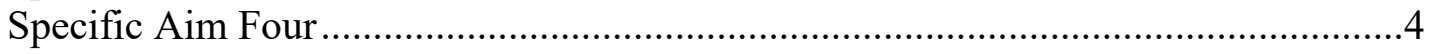

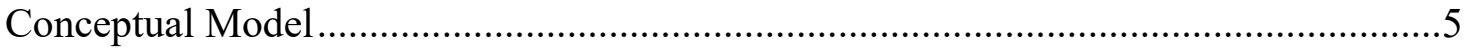

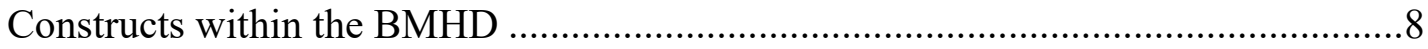

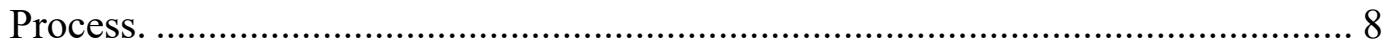

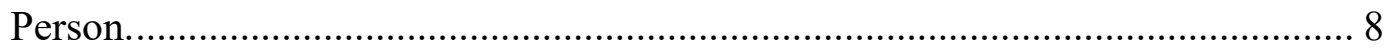

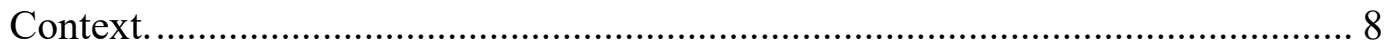

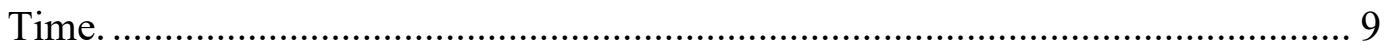

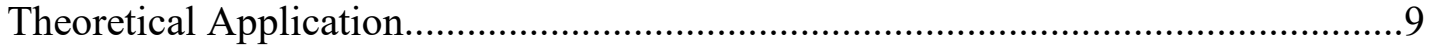

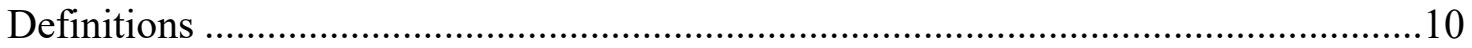

Health-Related Quality of Life ...................................................................... 10

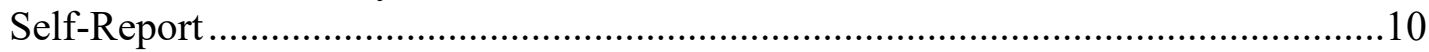

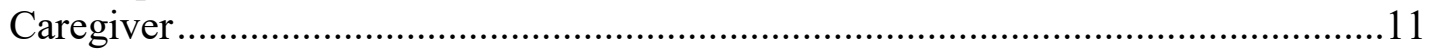

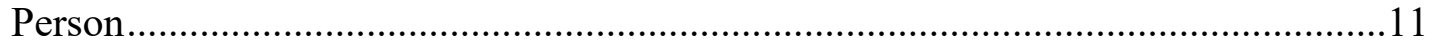

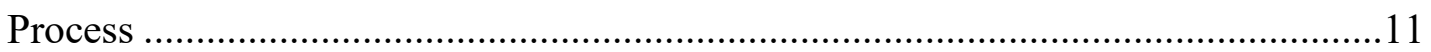

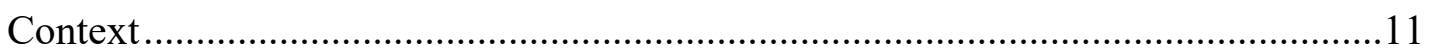

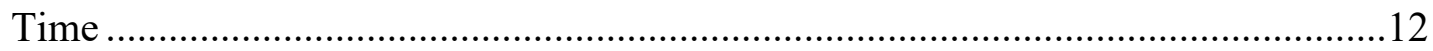

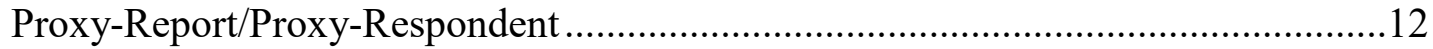

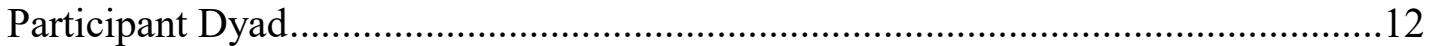

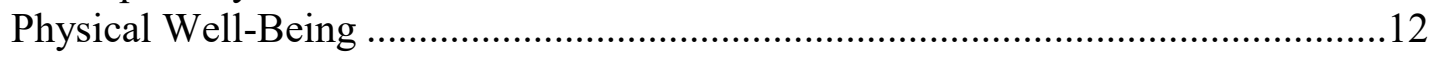

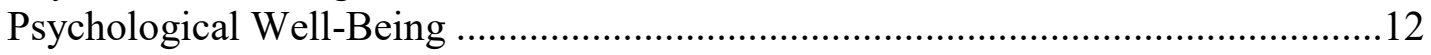

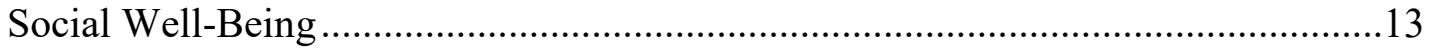

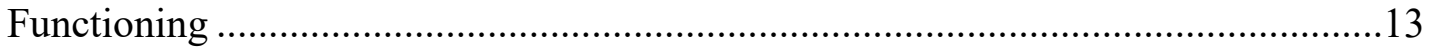

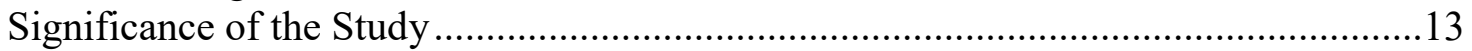

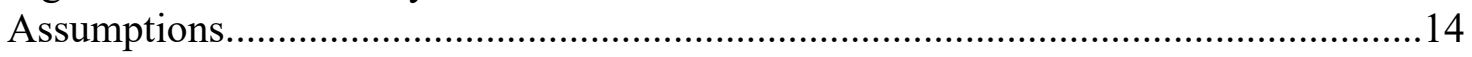

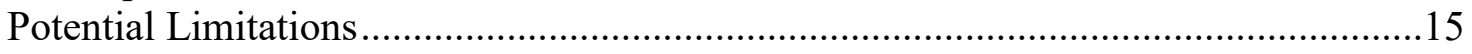

CHAPTER 2. REVIEW OF LITERATURE REVIEW .......................................16

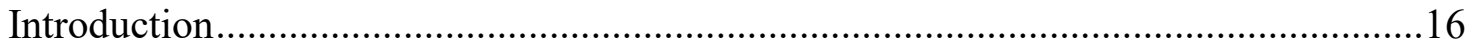

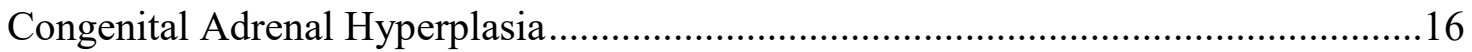

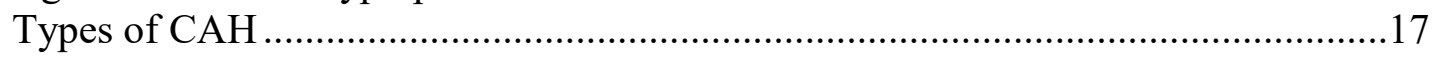

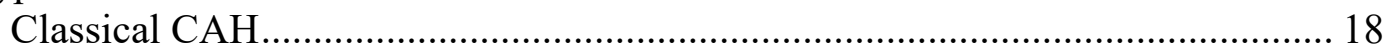

Classical Salt-Wasting CAH...................................................................... 18

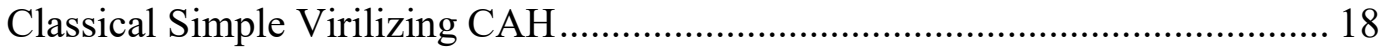

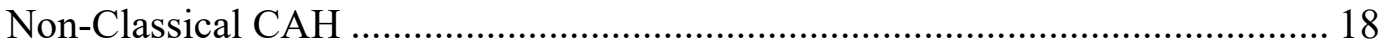

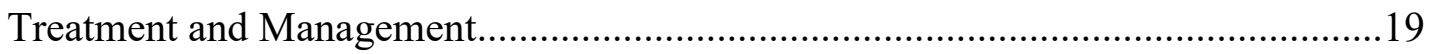

Effects of Under- and Over-Treatment of CAH ....................................................19 
Obesity, Metabolic Syndrome, and Cardiovascular Disease. ............................ 19

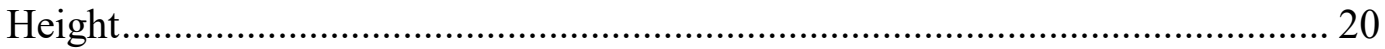

Reproductive Function, Infertility, and Pregnancy ............................................ 21

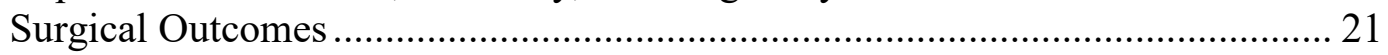

Quality of Life versus Health-Related Quality of Life ............................................21

Health-Related Quality of Life Research in Patients with $\mathrm{CAH}$...............................22

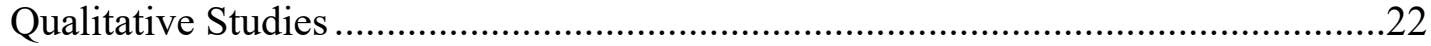

Quantitative Studies ...................................................................................2 27

Bioecological Model of Human Development ............................................................29

Factors within the BMHD Model Identified in HRQOL Literature ..........................29

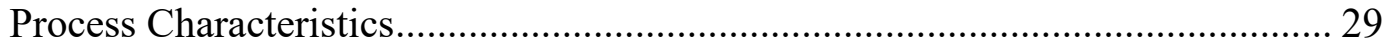

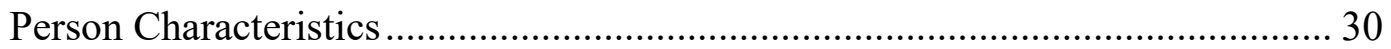

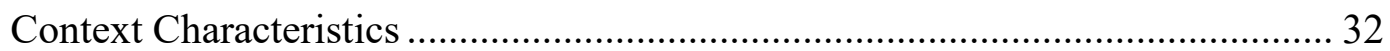

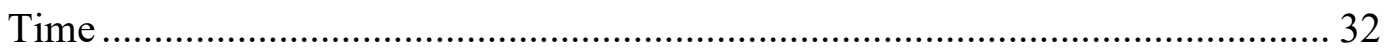

Measuring Health-Related Quality of Life in Children with CAH ............................32

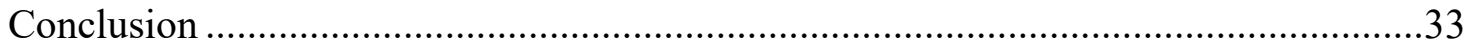

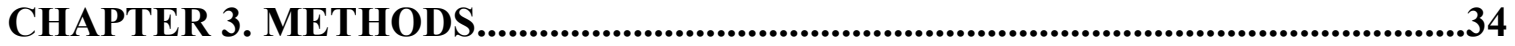

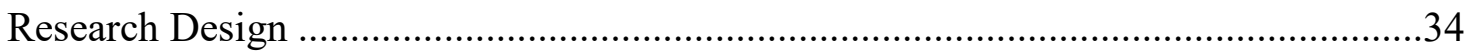

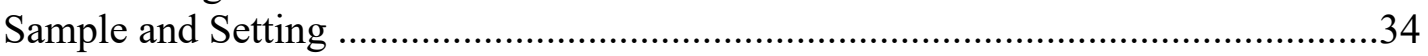

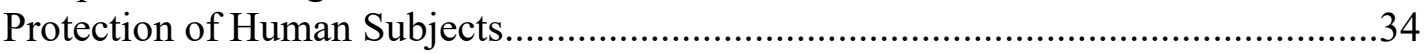

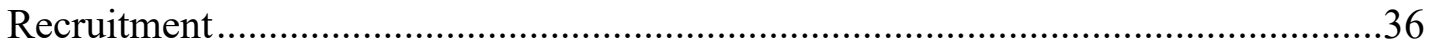

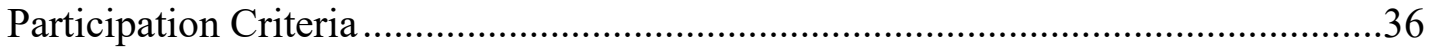

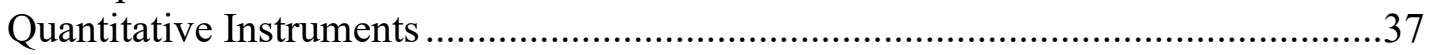

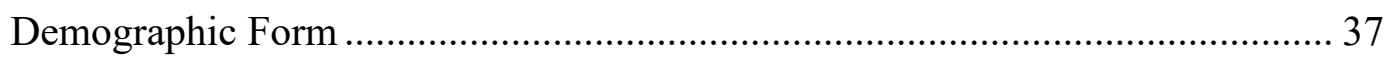

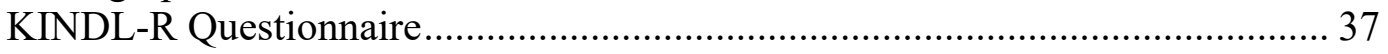

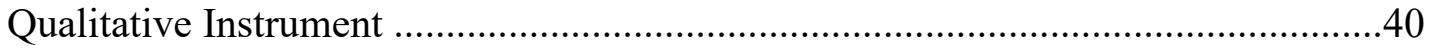

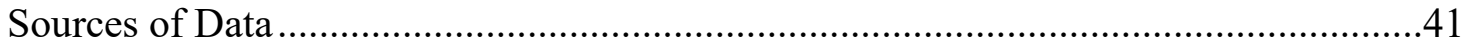

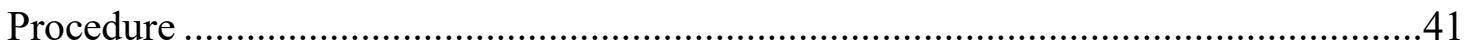

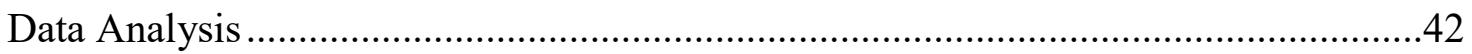

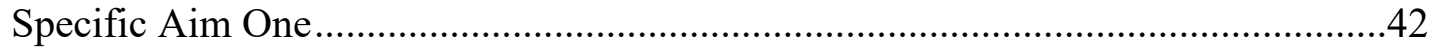

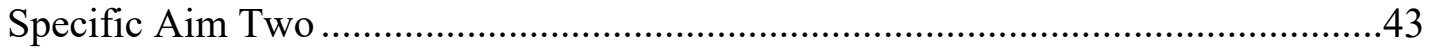

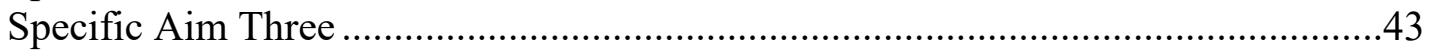

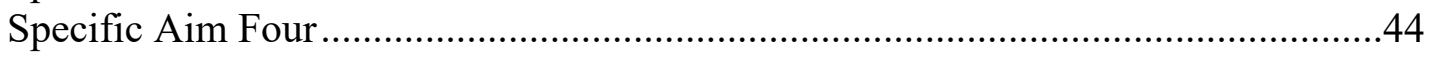

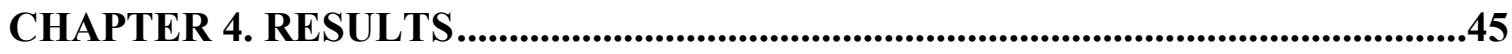

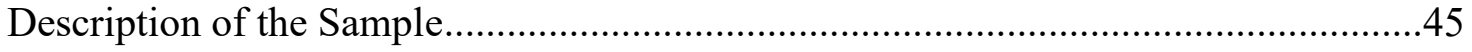

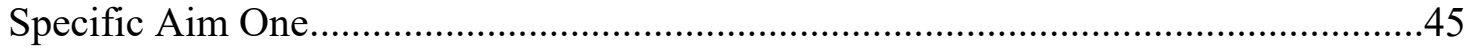

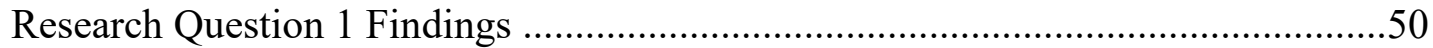

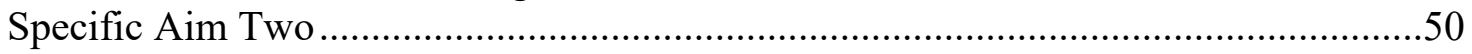

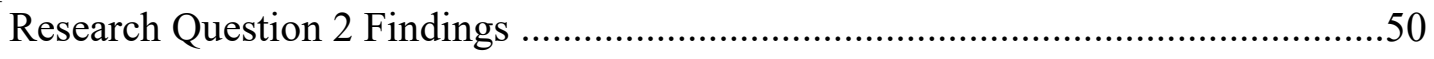

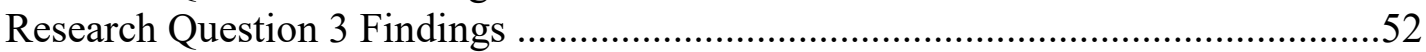

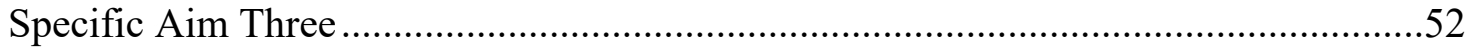

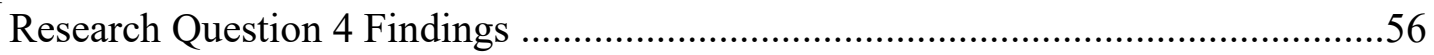

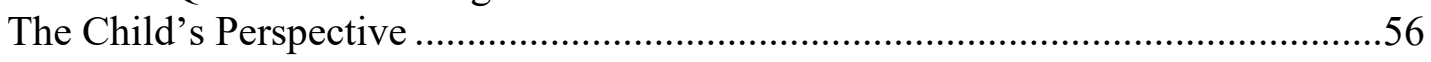

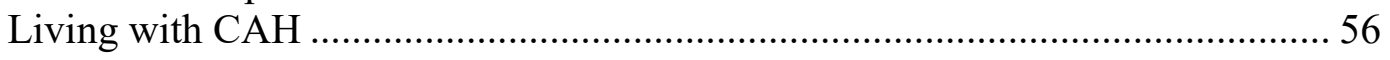




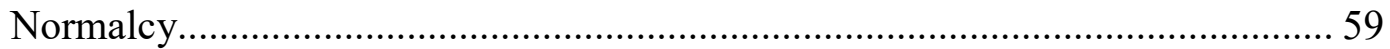

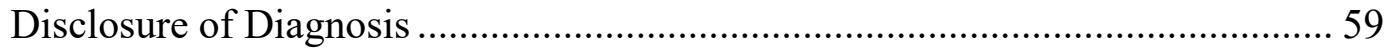

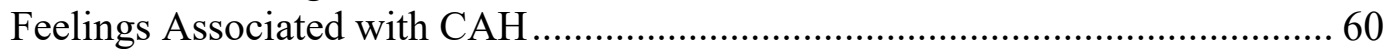

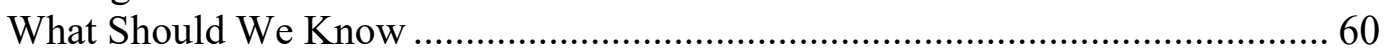

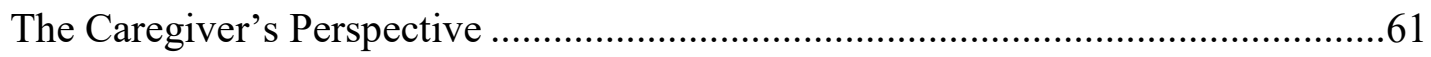

Living with $\mathrm{CAH}$

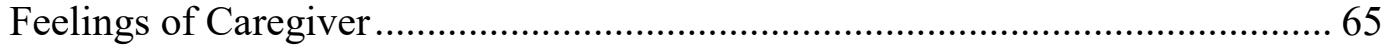

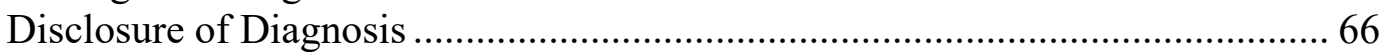

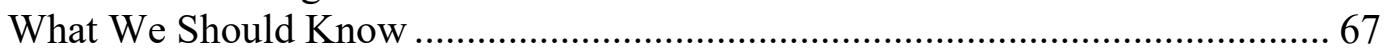

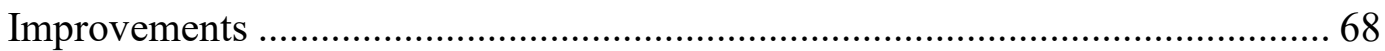

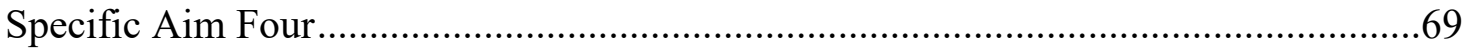

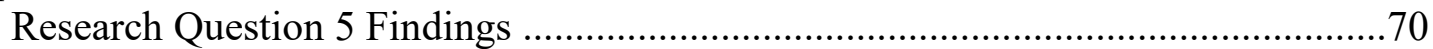

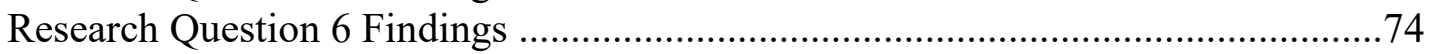

Research Question 7 Findings .............................................................................

CHAPTER 5. DISCUSSION .....................................................................................78

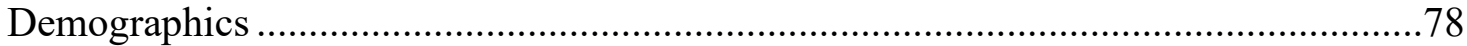

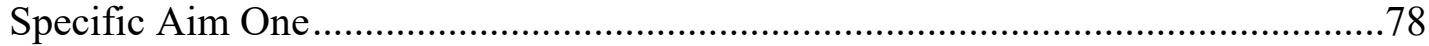

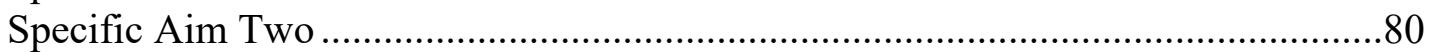

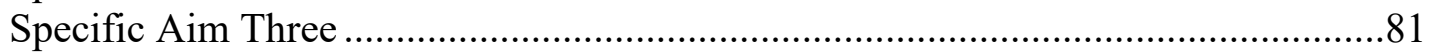

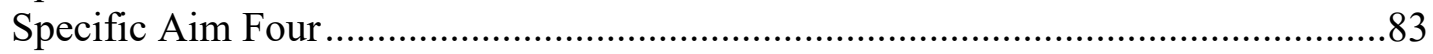

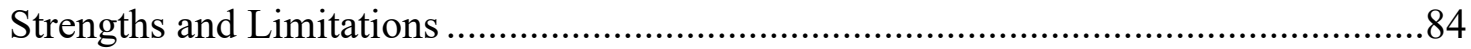

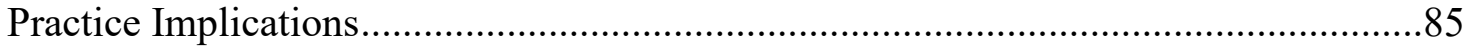

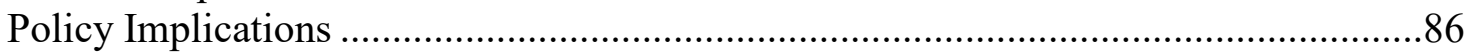

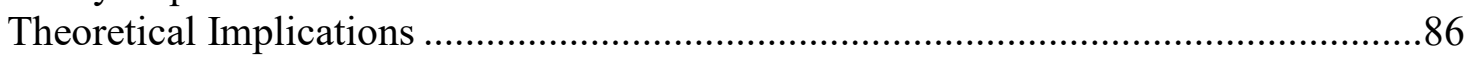

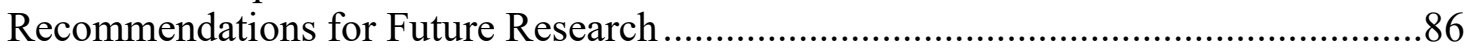

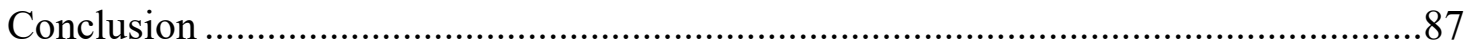

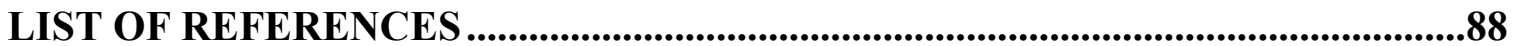

APPENDIX A. APPROVED CONSENT FORM FOR CHILDREN WITH CAH...97

APPENDIX B. CERTIFICATE OF CONFIDENTIALITY ........................................105

APPENDIX C. PARTICIPANT FLYER................................................................108

APPENDIX D. PARTICIPANT INTEREST LETTER..............................................109

APPENDIX E. DEMOGRAPHIC FORM........................................................................110

APPENDIX F. KINDL-R CHILDREN'S QUESTIONNAIRE ...................................116

APPENDIX G. ADOLESCENT KINDL-R QUESTIONNAIRE ................................119

APPENDIX H. PARENT KINDL-R QUESTIONNAIRE ............................................122

APPENDIX I. INTERVIEW GUIDE FOR CHILD/ADOLESCENT .......................125 
APPENDIX J. INTERVIEW GUIDE FOR CAREGIVER/PARENT..

APPENDIX K. ONLINE SURVEY CONSENT ............................................................131

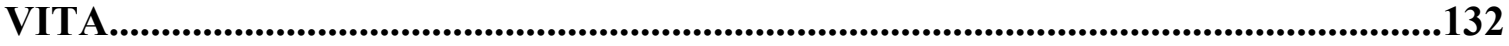




\section{LIST OF TABLES}

Table 1-1. Similarities among the BMHD, HRQOL, and the KINDL-R

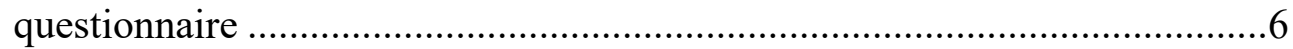

Table 2-1. Summary of studies on HRQOL of patients with CAH and DSD...............23

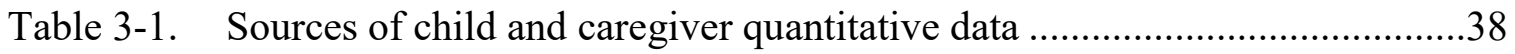

Table 4-1. Demographics and health characteristics of children with CAH................46

Table 4-2. Demographic characteristics of the caregivers .......................................48

Table 4-3. KINDL-R health related quality of life scores of female children with CAH obtained via self-report $(n=25)$ and caregiver proxy-report $(n=25) \ldots 51$

Table 4-4. Associations between demographic health characteristics and HRQOL rating scale.

Table 4-5. Association between demographic health characteristics and diagnosis ......54

Table 4-6. Associations between demographic health characteristics and frequency of communication.

Table 4-7. Comparison of the child QUAN interview data and the QUAL KINDLR scores

Table 4-8. Comparison of the caregiver QUAN interview data and the QUAL KINDL-R scores 


\section{LIST OF FIGURES}

Figure 1-1. Bronfenbrenner's Bioecological Model of Human Development .................7

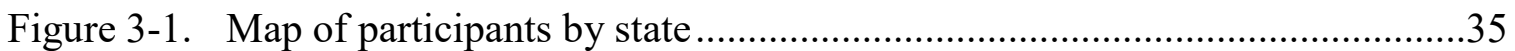

Figure 4-1. Mixed methods convergent design of HRQOL of female children with

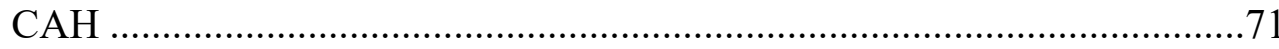




\section{LIST OF ABBREVIATIONS}

210HD

AGFI

AI

BMHD

BMI

CARES

$\mathrm{CAH}$

CBCL

CDC

DSD

ED

EMS

HADS

GBB-24

HRQOL

KINDL-R

PAI

PCOS

QOL

QUAL

QUAN 21-hydroxylase deficiency

Adjusted Goodness of Fit Index

Adrenal Insufficiency

Bioecological model of human development

Body Mass Index

Congenital Adrenal Hyperplasia Research Education and Support Foundation

Congenital adrenal hyperplasia

Child Behavior Checklist

Centers for Disease Control and Prevention

Disorder of Sexual Development

Emergency Department

Emergency Medical Services

Hospital Anxiety and Depression Scale

Giessan Subjective Complaints List

Health-related quality of life

KINDL-R Questionnaire for Measuring Health-Related Quality of Life in Children and Adolescents

Partial androgen insensitivity

Polycystic Ovary Syndrome

Quality of life

Qualitative

Quantitative 
RMSEA

SF-36

SOP

UTHSC IRB
Root Mean Square Residual

36- Item Short Form

Standard Operating Procedure

University of Tennessee Health Science Center Institutional Review Board 


\section{CHAPTER 1. INTRODUCTION}

\section{Overview}

Health-related quality of life (HRQOL) has become a meaningful area in research, medical management, and in the evaluation of health outcomes. Prior to the 1980s, the criteria for evaluating health outcomes focused on symptoms and physiologic indicators of health (i.e., lab values, clinical observations, or function tests). However, during the past two decades there has been a shift in perspectives to include patient-oriented outcomes (Ravens-Sieberer et al., 2006). The term HRQOL reflects this shift in perspective. Therefore, not only are symptomatology and clinical indicators important, but equally important is how patients view their health.

Health-related quality of life is a multidimensional concept that provides insight into individuals' perceived health and well-being. It emerged from the concept quality of life (QOL), which encompasses domains that essentially describe individuals' state of well-being and life satisfaction (World Health Organization, 2005). Health-related quality of life was developed to omit attributes not associated with health and to narrow the focus to the effects of illness, health, and treatment on QOL (Ferrans, Zerwic, Wilbur, \& Larson, 2005). Compared to standard medical outcomes, HRQOL takes into account an individuals' objective and subjective perspectives. The objective perspective emphasizes what individuals believe they can do. In contrast, the subjective perspective emphasizes the individuals" "appraisal" of HRQOL (Eiser \& Morse, 2001b).

Moreover, HRQOL focuses on domains of physical, psychological, and social well-being and function as perceived by the individuals (Matza, Swensen, Flood, Secnik, \& Leidy, 2004; Ravens-Sieberer et al., 2001). The physical domain refers to subjective beliefs about physical symptoms and sensations, and pain or bodily pain. This domain also includes individuals' satisfaction with their own physical condition. The psychological domain refers to negative or positive feelings, self-esteem and body image, and cognitive behavior. The social domain refers to individuals' beliefs and feelings about their friends, school, and family/home (Rajmil et al., 2004).

There is some evidence that suggests adults and children report their HRQOL within the same domains (Bullinger, Schmidt, Petersen, \& Ravens-Sieberer, 2006). Yet, the specific aspects of each domain are different. In children, HRQOL exists on a developmental continuum. Thus, certain aspects of HRQOL depend on the developmental stage of the child. Taylor and colleagues proposed that children experience many developmental stages; therefore, children's feelings and behaviors toward the illness and treatment may often be influenced by social contexts, cultural contexts and personal characteristics (Taylor, Gibson, \& Franck, 2008a). Although children experience many developmental stages of life, there is insufficient evidence on how these stages, and other factors influence HRQOL, particularly in children with disorders such as congenital adrenal hyperplasia (CAH). The lack of evidence delays 
both research and health care by overlooking specific issues that are relevant to children with chronic or long-term conditions (Taylor et al., 2008a).

In children with CAH, HRQOL is a long-neglected subject. Furthermore, CAH was not described until the mid- $19^{\text {th }}$ century, and it was almost a decade later before researchers understood and recognized the genetic nature and hormonal abnormalities that were associated with CAH (White \& Speiser, 2000). Researchers first described $\mathrm{CAH}$ as group of inherited enzyme deficiency disorders that impair normal steroid synthesis usually caused by a 21-hydroxylase deficiency (21OHD) (New, 2004). It is now categorized as classical and non-classical $\mathrm{CAH}$, occurs in both males and females, and is more prominent in certain racial (i.e., Caucasian) and ethnic (i.e., Jewish) populations (Zainuddin, Grover, Shamsuddin, \& Mahdy, 2013). In children with classical CAH, excessive androgen production in utero causes external genital virilization, which presents as ambiguous genitalia in newborn girls. In such cases, surgery is often needed to correct the external genitalia. Approximately $75 \%$ of individuals with classical CAH also have severe aldosterone deficiency, which is known as salt-wasting CAH. The remaining $25 \%$ of individuals with classical CAH produce sufficient amounts of aldosterone and therefore escape the salt wasting crisis. This is known as simple virilizing CAH (Merke \& Poppas, 2013; New, 2004; Nimkarn, Lin-Su, \& New, 2011; Riepe \& Sippell, 2007; Speiser et al., 2010). In non-classical CAH, children exhibit a mild to moderate reduced synthesis of cortisol. Girls with non-classical CAH are not virilized at birth and do not have ambiguous genitalia (Demirci \& Witchel, 2008; Riepe \& Sippell, 2007).

Prior QOL research in individuals with CAH typically focused on optimizing medical treatment and outcomes. Specifically, research focused on the best medical regime such as the use of long-term glucocorticoids and mineralcorticoid replacement and the consequences of over and under treatment (Kochar \& Jindal, 2011; Turcu \& Auchus, 2015). Other areas of treatment and outcomes focused on ideal age for genital surgery, and/or metabolic consequences of CAH (Kochar \& Jindal, 2011; Witchel \& Azziz, 2011). Although treatment has focused on optimizing the HRQOL of individuals with $\mathrm{CAH}$, management is complex because there are many aspects to care. Retrospective studies in adult females with CAH suggest that HRQOL is influenced by personal factors such as genital ambiguity, impaired growth, higher incidence of obesity, metabolic syndrome, hypertension, acute adrenal crisis, as well as losses in psychosexual milestones (Gilban, Alves-Junior, \& Beserra, 2014). Other studies reveal that CAH also impacts HRQOL in other domains such as psychological functioning (Bennecke, Grüters, Köhler, Thyen, \& Lux, 2017; Berenbaum, Korman Bryk, Duck, \& Resnick, 2004; Malouf, Inman, Carr, Franco, \& Brooks, 2010). Psychological characteristics such as coping and adaptation and social contexts such as family have been identified as factors that contribute to individuals' perceived HRQOL (Bullinger \& Ravens-Sieberer, 1995). Today, there is some agreement that non-disclosure, shame, secrecy, and stigmas associated with having ambiguous genitalia, surgery to normalize the genitalia, and repeated genital examinations all place a burden on the psychological well-being of individuals with $\mathrm{CAH}(\mathrm{Cull}, 2005)$. Although, there has been a shift in perspectives to 
include patient oriented outcomes, questions and data about the HRQOL of children with $\mathrm{CAH}$ were not brought to the forefront of research until recently.

Despite research efforts, it remains unclear which factors influence HRQOL and which factors are most relevant to female children with CAH. This indicates the need to go beyond identifying single factors, and address the complex relationship of factors that influence HRQOL and individuals' perceptions of these factors. Further research is needed to capture children's experiences and perceptions of living with $\mathrm{CAH}$ to adequately identify their needs and tailor interventions to improve their HRQOL.

\section{Purpose of the Study}

Research on the HRQOL of children with CAH is scarce. Most published research provides information about adults with $\mathrm{CAH}$. Furthermore, research studies about the HRQOL of adults with CAH primarily include: small sample sizes, a crosssectional design, a limited number of factors that may influence HRQOL, and clustering individuals with $\mathrm{CAH}$ with other disorders such as disorders of sexual development (DSD). Despite progress in improving the HRQOL of adults with CAH, there is no reason to assume that implications for measuring HRQOL should be limited to adults. In fact, due to the nature of the disorder recent research suggests that children with $\mathrm{CAH}$ may experience lower scores in the physical, psychological, and social domains of HRQOL (Gilban et al., 2014).

Given the current state of knowledge of HRQOL in children diagnosed with $\mathrm{CAH}$, the purpose of this study is to examine the HRQOL of female children with $\mathrm{CAH}$ as reported by female children and their caregivers. A convergent mixed methods design will be used to identify factors (i.e., process, person, context, demographic characteristics) that may affect HRQOL of female children with CAH, compare measurements of HRQOL based on self-report and caregiver proxy-report, and to explore associations between HRQOL and factors that may influence HRQOL.

\section{Specific Aims and Research Questions}

The following specific aims were developed to examine the HRQOL of female children and adolescents with $\mathrm{CAH}$.

\section{Specific Aim One}

The first specific aim was to measure the HRQOL of female children with CAH. The following research question pertains to the first specific aim:

Research Question 1: What is the HRQOL of female children with CAH as reported by: 

a. children with CAH?
b. caregivers of children with $\mathrm{CAH}$ ?

\section{Specific Aim Two}

The second specific aim was to examine the differences and associations between child self-report and caregiver proxy-report of the HRQOL of female children with CAH.

Research Question 2: Are there any differences between child self-report and caregiver proxy-report KINDL-R scores?

Research Question 3: Are there any associations between demographic health factors and HRQOL graphic rating scale scores as reported by caregivers of female children with CAH?

\section{Specific Aim Three}

The third aim was to explore the HRQOL and factors that contribute to HRQOL of female children with $\mathrm{CAH}$ from the perspectives of children with $\mathrm{CAH}$ and their caregiver.

Research Question 4: What themes on HRQOL and factors that may contribute to the HRQOL of children with CAH emerged during interviews with:
a. children with CAH?
b. caregivers of children with CAH?

\section{Specific Aim Four}

The last aim was to compare the quantitative and qualitative child and caregiver results on HRQOL of female children with CAH.

Research Question 5: To what extent do the themes on HRQOL emerging from interviews with children support the self-reported HRQOL as measured by the KINDL-R?

Research Question 6: To what extent do the themes on HRQOL emerging from interviews with caregivers support the caregiver proxy-reported HRQOL as measured by the KINDL-R? 
Research Question 7: To what extent do the themes on HRQOL emerging from interviews with children and their caregivers support the self-report and caregiver proxy-reported HRQOL as measured by the KINDL-R?

\section{Conceptual Model}

To date, there are limited studies that examine the HRQOL of children with CAH, and none used a conceptual framework to understand children's experiences and perceptions of HRQOL. For this reason, the bioecological model of human development (BMHD) was used as a guide to examine HRQOL in children with CAH. The BMHD primarily focuses on development as an outcome. Within this model, development is conceptualized as the physical, cognitive, and social change of both an individual and/or group throughout the life course (Bronfenbrenner, 2004; Bronfenbrenner \& Morris, 2006). In children with chronic disorders such as $\mathrm{CAH}$, development is important because it: 1) affects children's understanding and perception of their health, illness, and subjective treatment; 2) affects important areas of functioning; and 3) is attached to varying areas of concern such as disease visibility (Koot, 2014; Taylor, Gibson, \& Franck, 2008b). According to Taylor et al. (2008b), "the relationship between life and illness cannot be seen in isolation of development" (p. 1831). Thus, development is positively associated with well-being and HRQOL. Table 1-1 displays the similarities among the BMHD, HRQOL, and KINDL-R constructs and concepts of development as well as the proposed factors that influence development and HRQOL. Thus, the BMHD was used in this study because it takes into consideration the objective and subjective developmental constructs as well as individual experiences, feelings, and personal beliefs that contribute to children's HRQOL.

The BMHD proposes that there are multiple stages and layers of development. Therefore, development is influenced not by a single construct, but by several constructs that accompany the individual throughout the life course. The BMHD identifies these developmental constructs as: proximal processes, personal characteristics, contextual characteristics (environment) and time (Bronfenbrenner \& Morris, 2006). Figure 1-1 is a modified version of the BMHD that illustrates the four factors that may influence the HRQOL of individuals with CAH.

Moreover, the BMHD suggests that development occurs through reciprocal interactions between two individuals, objects, and symbols within the immediate environment. Interactions are not limited to people, but can also be things that invite exploration, attention, or imagination. According to the BMHD, successful development occurs when the reciprocal interactions occur consistently over time. This is known as proximal processes. Proximal processes are not unidirectional. Therefore, the "form, power, content, and direction of the proximal processes effecting development vary" according to the personal characteristics of the individual, the environment in which the processes take place, and the time in which the processes are occurring (Bronfenbrenner \& Morris, 2006, p. 798). For example, successful development is a result of parent-child or child-peer interactions and relationships (proximal processes), personal characteristics 
Table 1-1. Similarities among the BMHD, HRQOL, and the KINDL-R questionnaire

\begin{tabular}{llll}
\hline BMHD Constructs & BMHD Concepts & HRQOL & KINDL-R Subscales \\
\hline Process & $\begin{array}{l}\text { Social change of } \\
\text { individual } \\
\text { Physical, } \\
\text { cognitive }\end{array}$ & $\begin{array}{l}\text { Social well-being } \\
\text { Person }\end{array}$ & $\begin{array}{l}\text { Family, Friends } \\
\text { Psychological well- } \\
\text { being }\end{array}$ \\
Time & Social well-being & $\begin{array}{l}\text { Physical well-being, } \\
\text { Self-Esteem, }\end{array}$ \\
& & $\begin{array}{l}\text { Emotional well- } \\
\text { being } \\
\text { Everyday } \\
\text { Functioning }\end{array}$ \\
& & $\begin{array}{l}\text { Different versions to } \\
\text { account for the } \\
\text { developmental age } \\
\text { differences. }\end{array}$ \\
\hline
\end{tabular}

Note. BMHD = bioecological model of human development; HRQOL = health-related quality of life; KINDL-R = KINDL-R Questionnaire for Measuring Health-Related Quality of Life in Children and Adolescents. 


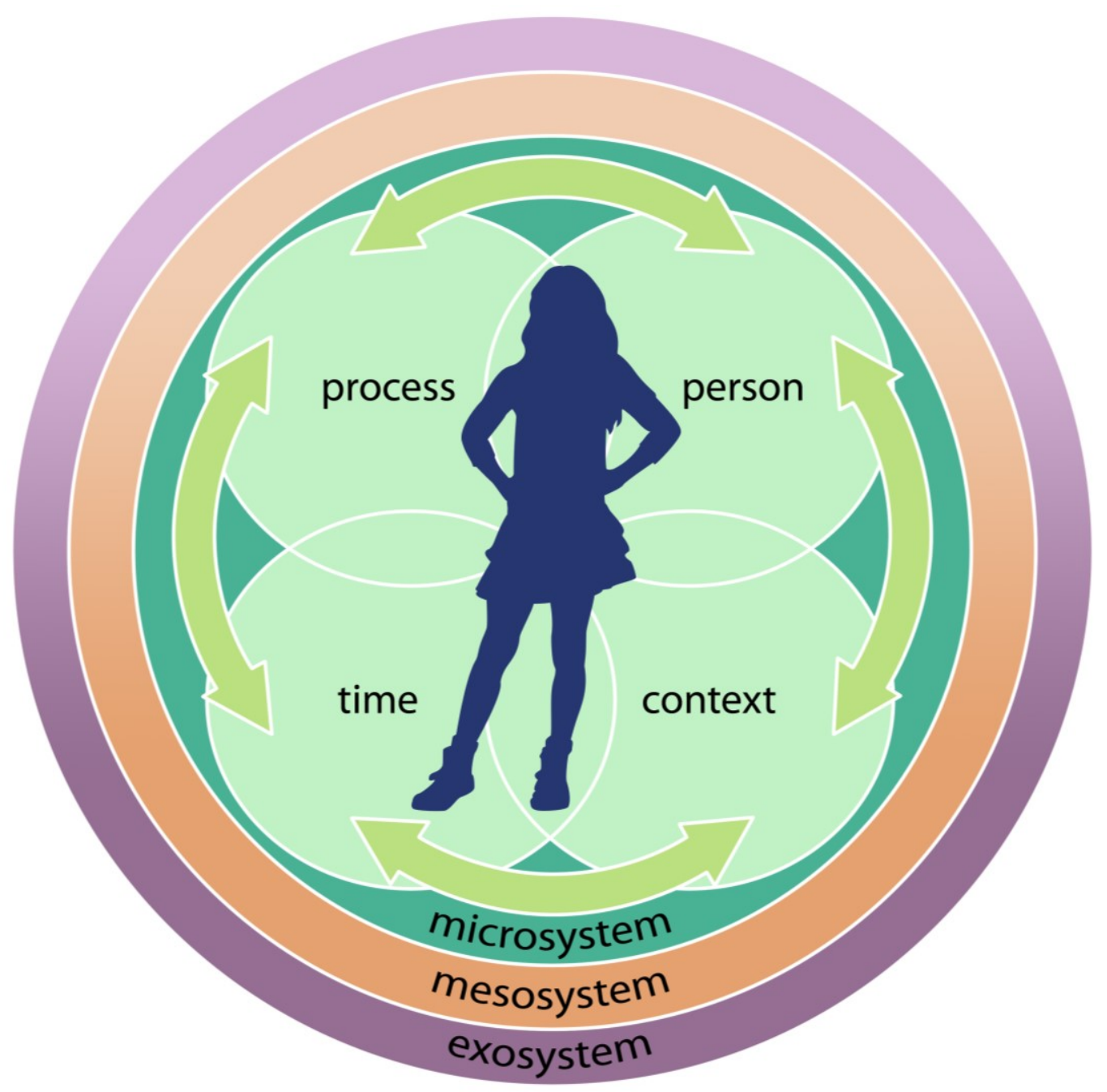

Figure 1-1. Bronfenbrenner's Bioecological Model of Human Development Sources: Bronfenbrenner, U. (2004). Making Human Beings Human: Bioecological Perspectives on Human Development. The SAGE Program on Applied Developmental Science: SAGE Publications (CA); and Bronfenbrenner, U., \& Morris, P. (2006). The Bioecological Model of Human Development in R.M. Lerner (Ed.), Handbook of Child Psychology: Theoretical Models of Human Development (Vol. 1, pp. 793-828): John Wiley \& Sons, Inc. 
of the child, the environment in which the relationships are fostered, and the life stage of the child (Bronfenbrenner \& Morris, 2006).

\section{Constructs within the BMHD}

The BMHD contains four constructs — process, person, context, and time - which are believed to influence outcomes. The examination of these constructs justifies the importance of examining a phenomenon such as HRQOL in its respective context to children with $\mathrm{CAH}$.

Process. Process, specifically proximal processes, are forms of interactions that occur between the developing individual and people, objects, or symbols within the immediate environment. Bronfenbrenner believes that proximal processes are the driving force in human development. Examples of proximal processes are the child-child activities, activities between a parent-child, problem solving, and acquiring new knowledge. The primary people the child interacts with over time are usually parents or caregivers. However, as the child gets older, these interactions may include siblings, peers, friends, or mentors (Bronfenbrenner \& Morris, 2006).

Person. The construct of personal characteristics or person represents the biological, cognitive, emotional, and behavioral characteristics an individual brings into any social setting (Bronfenbrenner \& Morris, 2006). Person is the most distinguished construct of the model because of its ability to affect direction and strength of the proximal processes. Person is composed of three characteristics - demand, resource, and force (Tudge, Mokrova, Hatfield, \& Karnik, 2009). Demand characteristics are described as those that act as an "immediate stimulus" such as age, gender, skin color, and physical appearance (Tudge et al., 2009). Such characteristics can welcome or discourage reactions from the social setting, which affects the way proximal processes are developed (Rosa \& Tudge, 2013). Resource characteristics are associated with mental and emotional resources that influence the ability to interact effectively in proximal processes such as past experiences, skills, and intelligence. Resource characteristics are required to successfully function at any stage of development. Force characteristics include different levels of temperament, motivation, and persistence (Tudge et al., 2009). Force characteristics have the ability to initiate and/or sustain proximal processes (Bronfenbrenner \& Morris, 2006).

Context. Context characteristics or context is a group of nested systems within the immediate and remote environment. The four systems include: microsystem, mesosystem, exosystem, and macrosystem. The microsystem describes the relationship between the developing person and the immediate environment. The mesosystem consists of the interactions between two or more settings (i.e., home and school). The exosystem consists of the interactions between two settings in which one of the settings does not contain the developing person, yet it indirectly affects the settings in which the developing person lives. For example, a father has been stressed at work, and as a result is more irritable than usual with his child when he gets home. The father's work is an 
exosystem for the child. Although the child spends no time there, it indirectly impacts the child through its effects on the father. The macrosystem includes any social structures or groups whose members share similar values or belief systems, resources, lifestyles, and patterns of social interchange (Bronfenbrenner, 1994; Tudge et al., 2009).

Time. Time is what happens across the lifespan. In the same way that context was divided into levels, so is time. Micro-time refers to what is happening during a specific moment or interaction. Meso-time is the degree that activities and interactions transpire consistently in individuals' environment - usually across broader intervals such as days and weeks. Macro-time refers to the notion that development varies according to historical events that are happening as individuals are at specific age (Tudge et al., 2009).

The BMHD can be used to explore a variety of outcomes including health outcomes. The BMHD is used as a framework in this study to better understand the HRQOL of female children with CAH. Although the BMHD model has not been used to explore HRQOL in children with $\mathrm{CAH}$, examining key constructs may provide insights that lead to improvement and delivery of care in a developmentally appropriate way that yield a higher HRQOL.

\section{Theoretical Application}

Similar to development, HRQOL contains the same domains- physical, social, psychological- that describe development within the BMHD Model. Likewise, HRQOL as an outcome is influenced by several constructs that accompany individuals throughout their life course. The aforementioned constructs-process, person, context, time-are also significant in reference to female children with CAH and to HRQOL as explored in this study.

The concept of process is important because female children with CAH interact with specific people — parents, relatives, healthcare providers, and peers — on a regular basis over the course of their lifetime. Engaging in these interactions over time not only provides children with knowledge, but it also motivates children to participate in activities with others and on their own (Bronfenbrenner \& Morris, 2006). For example, gradual, more complex interactions between children and parents are likely to result in children becoming agents of their own health. Thus, the construct is applicable in that it allows us to understand how these processes between children and caregivers or between children and their peers influence HRQOL in female children with CAH. The construct of processes may also help to identify relationships that are likely to hinder or improve HRQOL.

The construct of person is significant in its application to female children with $\mathrm{CAH}$. This construct justifies the importance of biological and genetic characteristics, as well as psychological, emotional, and social characteristics that can be used to better understand factors that influence HRQOL. While personal characteristics are typically 
unchangeable, they may be helpful to address issues and tailor interventions for specific groups (Ferrans et al., 2005).

Context, another important construct in the application of HRQOL in female children, allows us to understand the extent that the environment in which children interact influences HRQOL. Female children with CAH are nested within multiple contexts including the family, peer group, classroom, and the community. Each of these contexts may contribute to the HRQOL of female children by mediating the effects of the disorder and treatment (Matza et al., 2004).

Time also plays an important role in the application of this theoretical model. HRQOL may vary according to specific events that occur in children's lives. HRQOL of female children with $\mathrm{CAH}$ might also vary according to current or historical events that occur during the lifespan. For example, children with classical CAH who had surgery younger in life may perceive their HRQOL differently than female children without surgery. Children's experiences may influence the current policies regarding delayed surgery in children with similar conditions.

\section{Definitions}

The literature provided definitions for the major concepts and variables examined in this study. Following are the definitions of these concepts used within this study.

\section{Health-Related Quality of Life}

Health-related quality of life is a multidimensional concept that includes domains of physical, psychological, and social well-being and function as perceived by the individual (Ravens-Sieberer et al., 2001). It emerged from the concept quality of life (QOL), which encompasses domains that essentially describe an individual's state of well- being and life satisfaction (WHO, 2005). HRQOL is operationally defined by overall score of each subscale of the KINDL-R Questionnaire (Bullinger, Brutt, Erhart, Ravens-Sieberer, \& Group, 2008; Ravens-Sieberer \& Bullinger, 2000)

\section{Self-Report}

Self-report refers to individuals answering a question about themselves (Polit \& Beck, 2008). Children's perspectives are integral in this study in that they provide their subjective experience and perception of HRQOL, which may otherwise go unnoticed in situations when caregivers are the proxy respondents. Interviews were conducted with the children in this study. The KINDL-R Questionnaire has two self-report versions based on age. The KID-KINDL-R or Children's Version was administered to children 7-13 years 
and the Kiddo-KINDL-R or Teenagers Version was administered to adolescents 14-17 years (Raven-Sieberer \& Bullinger, 2000).

\section{Caregiver}

For the purposes of this study, caregiver is defined as an adult family member with whom children depend on to meet their emotional, behavioral, physical, and medical needs. Caregivers in this study are unpaid and are those that may care for an ongoing medical condition, a serious short-term condition, emotional, behavioral, or developmental problems (National Alliance for Caregiving, 2015). The BMHD supports the inclusion of caregivers through the concept of proximal processes. The interactions between caregivers and children are integral to investigating the HRQOL of a child with CAH. The KINDL-R Questionnaire has a Parent's Version for caregivers of children ages 7-17 years that was administered to the caregivers in this study (Ravens-Sieberer \& Bullinger, 2000).

\section{Person}

Person is a combination of characteristics such as child physical characteristics, abilities, and attitudes. Person is operationally defined by information obtained from the Demographic Form, interview data, and subscale and total scores of the KINDL-R Questionnaire (Bullinger et al., 2008; Ravens-Sieberer \& Bullinger, 2000).

\section{Process}

Process is the continuous interaction between children and their parents, peers, or other individuals across the life course. Process characteristics are the interpersonal influences on HRQOL such as friends, family, peers, and healthcare providers. Process is defined by information obtained from interview data and from the Friends and Family subscale scores of versions the KINDL-R Questionnaire (Bullinger et al., 2008; RavensSieberer \& Bullinger, 2000).

\section{Context}

Context is conceptualized as systems within the immediate and the remote environment. Context consists of settings such as the home, school, neighborhood, and work. Context is operationally defined by the information obtained from interview data and from the Everyday Functioning subscale scores of versions of the KINDL-R Questionnaire (Bullinger et al., 2008; Ravens-Sieberer \& Bullinger, 2000). 


\section{Time}

Time is what happens at specific moments during the life course. Time consists of days and weeks as well as what has happened in the past and present. Time is captured using data from the Demographic Form and interview.

\section{Proxy-Report/Proxy-Respondent}

Research involving children under of the age of 18 require the use of a proxyrespondent. The proxy-respondent is an individual who is familiar with the research participant and answers subjective questions about the child. This is called proxy-report (Polit \& Beck, 2008). The caregivers in this study participated as proxy-respondents for children with CAH. However, the HRQOL survey and interview data were collected from children and caregivers to determine the differences and associations between the child self-report and the caregiver proxy-report in this sample.

\section{Participant Dyad}

A participant dyad is a pair of individuals who participate in a research study together. Usually one person has a characteristic, such as a specific diagnosis, that is of interest to the researcher. In this study, participant dyads consisted of one child and the child's caregiver. Participant dyads were recruited to understand the association between child self-report and caregiver proxy-report of HRQOL of children with CAH, as well as child self-report and caregiver proxy-report of factors that influence HRQOL.

\section{Physical Well-Being}

Physical well-being in children is the ability to perform developmentally appropriate physical tasks in the absence of pain, physical limitations, and biological markers of disease (Capio, Sit, \& Abernethy, 2014). Physical well-being also refers to children's feelings about their physical health, as well as their feelings about energy and vitality (Rajmil et al., 2004). Conceptually, physical well-being is best represented by the construct of person, specifically the demand characteristics of a person. It is operationally defined by the Physical Well-Being subscale scores of the KINDL-R Questionnaire (Ravens-Sieberer \& Bullinger, 2000) as well as information obtained from the child and caregiver interviews.

\section{Psychological Well-Being}

Psychological well-being is a combination of signs that demonstrate the presence or absence of positive feelings about life, self-esteem, body image, and cognitive

behavior. Psychological well-being is measured through scores that reflect the presence 
of positive and negative effects as well as perceived overall life satisfaction (Keyes, 2002). Psychological well-being is operationally defined by information obtained from interview data and the Emotional Well-being, and Self-Esteem subscale scores of the KINDL-R Questionnaire (Ravens-Sieberer \& Bullinger, 2000). Conceptually, psychological well-being is best described as resource characteristics of developing individuals (Bronfenbrenner, 2004).

\section{Social Well-Being}

Social well-being refers to individuals feeling a sense of belonging at home, at school, with neighbors, in the community, and with other children (Bullinger et al., 2008). It is how individuals relate and function with others and with society (Cicognani, 2014; Keyes, 2002). Social well-being is operationally defined by information obtained from interview data and the Friend and Family subscale scores of the KINDL-R Questionnaire (Ravens-Sieberer \& Bullinger, 2000). Conceptually, social well-being is best represented by the constructs of process and context.

\section{Functioning}

Functioning is defined as functional status. Functional status is children's ability to perform daily activities. Measures of functioning gauge children's ability to perform specific tasks to meet basic needs and maintain health (Eiser \& Morse, 2001b; Wilson \& Cleary, 1995). Functional status is operationally defined by information obtained from the Demographic Form, interview data, and the Everyday Functioning subscale scores of the KINDL-R Questionnaire (Ravens-Sieberer \& Bullinger, 2000). Functioning is best conceptualized as resource characteristics of the developing person.

\section{Significance of the Study}

Measuring HRQOL as an outcome is relevant in the clinical setting. Compared to standard medical measures such as laboratory tests and clinical observations. HRQOL takes into account individuals' perceptions which are important to care (Ravens-Sieberer et al., 2006). Thus, measuring HRQOL may provide clinicians with greater knowledge and understanding of the quality and efficacy of treatment regimes, while also allowing individuals' subjective differences in treatments to be identified and hidden disorders to be revealed (Ravens-Sieberer et al., 2006). Likewise, the Centers for Disease Control and Prevention (CDC) proposes that measuring HRQOL could improve the overall health of the nation (CDC, 2013). According to the CDC, HRQOL as an outcome assists in decisions about the allocation of resources, determining the efficacy of treatments and interventions, and capturing data for policy decision-making (Cantrell \& Kelly, 2015; CDC, 2013). 
Furthermore, measuring HRQOL in children plays an important role as an outcome in health and economic infrastructures. Research suggests that the analysis of HRQOL surveillance data can identify subgroups, such as children with $\mathrm{CAH}$, with low perceived HRQOL and help to guide interventions to improve their circumstances (Jurgensen, Lux, Wien, Kleinnemeier, Hiort, \& Thyen, 2014). The publication of such data helps guide the development of strategic plans and evaluate the impact of community interventions (CDC, 2013). Moreover, literature on the HRQOL of children with CAH is scarce. As such, there is no conceptual framework for how we provide and manage care or literature that uses appropriate standardized instruments to measure HRQOL in children with intersex conditions such as CAH. This study seeks to build a foundation of literature needed to improve the HRQOL in female children with $\mathrm{CAH}$. The results of this study will provide additional knowledge that can be used to tailor and test interventions to improve the HRQOL of children with $\mathrm{CAH}$.

The importance of self-report is supported by Jokovic, Locker, and Guyatt (2004) who assert there are discrepancies between child report and caregiver report. Children may not always provide comprehensive information so caregivers are needed as a proxy. However, the extent to which parents understand how CAH affects their child's life remains questionable. Thus, discrepancies between child self-report and caregiver proxyreport may reveal new insights into differences in perspectives. This may be a result of parents' lack of awareness with respect to relationships and interactions that occur outside of the home. The importance of self-report is upheld by evidence conveying lower scores for domains in which caregivers and children have access to different information such as peer interactions (Jokovic, Locker, \& Guyatt, 2004). Investigating the associations between perspectives of caregivers and children with $\mathrm{CAH}$ will be informative to health care providers, and may encourage providers to consider both child and caregiver perspectives when making treatment decisions.

The information in this study will add to evidence about the care needed to improve HRQOL of female children with $\mathrm{CAH}$. The results of the study may provide valuable insights into the unique issues that children with $\mathrm{CAH}$ experience thus allowing for the development of therapeutic measures as well as approaches to assist children and families to further improve children's HRQOL.

\section{Assumptions}

1. The KINDL-R will be applicable to measure HRQOL in female children with $\mathrm{CAH}$.

2. The study design will allow for detection of differences between self-reported HRQOL of female children with $\mathrm{CAH}$ and that reported by caregivers.

3. Caregivers will overestimate certain aspects or subdomains of children's HRQOL.

4. The constructs within the Bioecological Model of Human Development are relevant to the HRQOL of female children with $\mathrm{CAH}$.

5. Interview data will provide greater insights into process characteristics. 
6. Children and caregivers' views of HRQOL as measured by the KINDL-R reflect proximal processes.

\section{Potential Limitations}

The potential limitations for the study are:

1. The sample size and characteristics of participants may limit the generalizability of the findings from this study.

2. The participants in the study will be recruited through non-probability convenience sampling methods, which may lead to bias and a non-representative sample. 


\section{CHAPTER 2. REVIEW OF LITERATURE REVIEW}

\section{Introduction}

Health-related quality of life (HRQOL) has become a popular construct in several disciplines including nursing, public health, epidemiology, psychology, and medicine. Across disciplines, research efforts have focused on conceptualizing, identifying, and measuring domains relevant to HRQOL and factors that influence HRQOL in individuals with chronic illnesses such as asthma and diabetes. Research findings reveal that regardless of the illness, there are several factors that influence an individual's perception within the physical, psychological, and social domains of HRQOL. In children with chronic illnesses, HRQOL is significant because many will not be cured, and the ramifications of the illness or treatment involve management well into adulthood (Cantrell \& Kelly, 2015).

Despite research efforts, outcome data on HRQOL in chronic illnesses, specifically in children with CAH, is rare. Several studies have examined HRQOL and factors that influence HRQOL in conditions similar to disorders of sexual development (DSD) and adrenal insufficiency (AI). However, significant gaps exist in CAH HRQOL literature, specifically related to children's perceptions and self-report of HRQOL, as well as factors influencing children's HRQOL. While several studies have described HRQOL and identified factors that influence HRQOL of adults with CAH, many have reported inconsistent results (Yau, Vogiatzi, Lewkowitz-Shpuntoff, Nimkarn, \& Lin-Su, 2015). These inconsistence delays the identification of other HRQOL factors most relevant to children with $\mathrm{CAH}$.

The purpose of this chapter is to synthesize the science on CAH and review published reports on HRQOL in children and adults with CAH. This chapter will also include the effects of over and under treatment of $\mathrm{CAH}$. Additionally, the bioecological model of human development (BMHD) will be used as a framework to identify factors that are associated with the HRQOL of children and adults with CAH. Lastly, the chapter will review concerns related to conceptualizing and measuring HRQOL in individuals with $\mathrm{CAH}$.

\section{Congenital Adrenal Hyperplasia}

Congenital adrenal hyperplasia $(\mathrm{CAH})$ refers to a group of autosomal recessive disorders of enzymatic deficiency disorders that impair synthesis of adrenal steroids. Normal levels of adrenal steroids are critical for normal fetal genital development, normal growth and development throughout childhood, maintenance of blood pressure, normal blood glucose levels, salt balance, and support of immune function throughout life. Dysfunction of the enzymes responsible for the synthesis of cortisol, aldosterone, and adrenal androgens can thus have far reaching implications. These implications are dictated by which enzyme is affected and to what degree synthesis is impaired. Generally 
speaking, however, children with CAH have an increase in adrenal androgens (steroid hormones that increase virilization) and may have decreases in cortisol (a stress hormone important for maintenance of blood sugar and blood pressure) and aldosterone (a salt balancing hormone).

Congenital adrenal hyperplasia can affect both males and females. The most common form has an increased prevalence rate in some geographical locations and ethnic groups, such as Ashkenazi Jews and Alaskan Yupiks (Speiser et al., 2010). CAH is an autosomal recessive disorder. Thus, in affected children, each parent typically harbors a change in the genetic material necessary for manufacture of a particular adrenal steroid. The parents may or may not have symptoms of this condition and thus they are known as carriers. The risk of two carriers having an affected child is 1 in every 4 pregnancies. An affected child inherits one copy of the changed gene from each parent (the changes can be different from each parent), with the net result of an enzyme in the affected child that is not functioning properly. This risk continues for each child born to the couple. If a woman has previously had a child with $\mathrm{CAH}$ and becomes pregnant again with the same partner, the fetus has a 25\% chance of having CAH (Speiser et al., 2010; White \& Speiser, 2000). Currently, many countries perform screening of infants for this condition and are able to identify and treat the most affected infants early. Newborn screening serves two purposes, namely identifying infants, especially males, with the classic form of 21-hydroxylase deficiency (21OHD) CAH who are at risk for life-threatening saltwasting crises and providing expeditious diagnosis of the affected female with ambiguous genitalia. Newborn screening rarely detects individuals with the non-classic form of 21-OHD CAH (Votava et al., 2005). Although newborn screening has significantly decreased the mortality of infants with $\mathrm{CAH}$, significant morbidities remain.

The most common form of CAH is caused by a deficiency of the enzyme responsible for the modification of a cholesterol moiety at the 21 st carbon position (21hydroxylase deficiency), accounting for more than 95\% of all cases (Allen et al., 1997; Lin-Su, Nimkarn, \& New, 2008; Speiser et al., 2010). Less common causes of CAH include the deficiencies of other enzymes responsible for progressive modifications to cholesterol including: 11-Beta-hydroxylase, 3-Beta-hydroxysteroid dehydrogenase, 17alpha-hydroxylase/17, 20-lyase, and steroidogenic acute regulatory protein (also called STAR) (Lin-Su et al., 2008).

\section{Types of CAH}

Congenital adrenal hyperplasia due to a 21OHD is categorized as classical or nonclassical CAH. Severe genetic mutations that impair the 21-hydroxylase enzyme result in the classical form of $\mathrm{CAH}$; while mild genetic mutations of the 21-hydroxylase enzyme result in the non-classical form of CAH (Merke \& Poppas, 2013). In all forms of CAH, children have cortisol deficiencies, and varying degrees of androgen excess (hyperandrogenism). However, approximately $75 \%$ of individuals who have classical $\mathrm{CAH}$ also lack the ability to synthesize sufficient aldosterone. This is referred to as salt- 
wasting $\mathrm{CAH}$. The remaining $25 \%$ of individuals with classical $\mathrm{CAH}$ produce sufficient amounts of aldosterone or have simple virilizing CAH (White \& Speiser, 2000).

Classical CAH. Females with classical CAH are exposed to excessive androgens in utero, leading to virilization of the genitalia and early identification at birth (Lin-Su et al., 2008; Speiser et al., 2010; White \& Speiser, 2000). The spectrum of virilization ranges from a nearly male-like appearance of the external genitalia with an enlarged phallus to minimal clitoromegaly. Other external genital findings include partially fused and ruggated labia majora, and a urogenital sinus in place of a separate urethra and vagina (Demirci \& Witchel, 2008).

In older children and adolescents, clinical symptoms of androgen excess include premature pubarche; the early development of facial, axillary, and pubic hair; as well as body odor and acne; and rapid growth and advancement of the bone age. Hirsutism (i.e., the excessive growth of coarse hairs in androgen dependent areas such as the face, chest, abdomen and back), menstrual abnormalities, short stature and overweight are often present in adolescents with classical CAH (Lin-Su et al., 2008).

Classical Salt-Wasting CAH. Children with the salt-wasting form of CAH cannot adequately synthesize aldosterone, and thus lack the ability to retain salt. Cortisol synthesis is also suboptimal, further complicating the abnormal salt and potassium levels with low blood glucose and negatively impacting blood pressure. Left untreated, infants with salt-wasting CAH may present with poor feeding, weight loss, failure to thrive, vomiting, dehydration, and metabolic acidosis, also known as an adrenal crisis (Nimkarn et al., 2011). This is a life-threatening situation for individuals with salt-wasting CAH as it increases the risk for hypovolemic shock and death (White \& Speiser, 2000). Before newborn screening for CAH was in place, male infants died from the adrenal crises associated with this condition (Allen et al., 1997).

Classical Simple Virilizing CAH. Females with simple virilizing CAH are also identified at birth as they have virilization of their genital area, but they produce sufficient amounts of aldosterone and cortisol, which allows them to bypass the saltwasting crisis. They also have abnormalities in their growth pattern with early pubertal development, hirsutism, and menstrual abnormalities (Lin-Su et al., 2008).

Non-Classical CAH. Compared to classical CAH, non-classical CAH due to $21 \mathrm{OHD}$ is mild and less severe. Females affected by non-classical CAH may be asymptomatic and can be diagnosed any time after birth. Affected females with nonclassical CAH primarily present with androgen excess and normal to mildly impaired cortisol production (Merke \& Poppas, 2013; Speiser et al., 2010). Similar to classical $\mathrm{CAH}$, females with non-classical CAH may have a variety of symptoms related to excess androgen production excluding ambiguous genitalia. Females with non-classical CAH may experience premature development of pubic or axillary hair, body odor, advanced bone age, and accelerated linear growth. Adolescent females with non-classical CAH may also have irregular or absent menses, polycystic ovaries, and alopecia (Lin-Su et al., 2008). 


\section{Treatment and Management}

Standard treatment of individuals with CAH seeks to correct the cortisol deficiency and suppress excessive androgen production by replacing deficient hormones (Merke \& Poppas, 2013; Nimkarn \& New, 2009; Speiser et al., 2010). In children with classical CAH, glucocorticoid doses that exceed physiological levels of cortisol secretion are given. Hydrocortisone is usually the choice of glucocorticoid therapy because it has a short half-life and is less likely to impair growth (Merke \& Poppas, 2013; Riepe \& Sippell, 2007; White \& Speiser, 2000). Additionally, mineralocorticoids such as fludrocortisone may be given to children with classical salt-wasting CAH to prevent excessive salt loss (Riepe \& Sippell, 2007). Adults and older adolescents with CAH may be treated with small doses of long-acting glucocorticoids like prednisone or dexamethasone (Han, Walker, Arlt, \& Ross, 2014).

In females with non-classical CAH, glucocorticoid therapy should be offered when symptoms of excess androgen are present (White \& Speiser, 2000). Low-dose glucocorticoid therapy may be used in children who have premature pubarche and advanced bone age that would significantly affect height. Treatment of non-classical CAH may also involve the use of oral contraceptives or anti-androgen agents to combat virilizing features (Speiser et al., 2010; White \& Speiser, 2000). In the event that oral contraceptive pills or anti-androgens are not preferred, females with non-classical CAH may choose to continue glucocorticoid therapy (Speiser et al., 2010). Furthermore, glucocorticoid therapy may be warranted during adulthood for infertility or recurrent miscarriages (White \& Speiser, 2000).

\section{Effects of Under- and Over-Treatment of CAH}

Management of $\mathrm{CAH}$ is complex because of adverse and long-term effects of glucocorticoid therapy. Early or over treatment of glucocorticoid therapy results in impaired growth in children, obesity, cardiovascular risk, and potential psychological effects (Merke \& Poppas, 2013). Likewise, under treatment causes progressive virilization, increased risk for adrenal crises, accelerated rates of bone maturation, early pubertal development, and short stature (Demirci \& Witchel, 2008).

Obesity, Metabolic Syndrome, and Cardiovascular Disease. Previously, adipose tissue (fatty tissue) was thought of as a storehouse for energy. However now it is understood that adipose tissue operates as an endocrine organ with several metabolic functions (Kim et al., 2016). This is of particular concern in individuals with CAH because researchers hypothesize that individuals with $\mathrm{CAH}$ have a higher risk of obesity (Völkl, Simm, Beier, \& Dörr, 2006). Researchers that conducted one of the first studies examining obesity in children with $\mathrm{CAH}$ reported a significant increase in obesity rates, and found a correlation between body mass index (BMI) and steroid dosage (Knorr \& Hinrichsen de Lienau, 1988). Ten years later, Cornean, Hindmarsch, and Brook (1998) assessed BMI and adiposity rebound - the age in which BMI increases in childhood. Earlier adiposity rebound is associated with an increased risk for development of obesity 
in adolescence. Typically, in children, this occurs after 3 years of age. Results of this study determined that adiposity rebound in children with CAH took place at approximately 1.74 years of age (Cornean et al., 1998).

Other studies report that the bone mineral density, lean body mass, and fat mass are significantly higher in young adults with $\mathrm{CAH}$ compared to the normal population (Cornean et al., 1998; Mooij, Kroese, Claahsen-van der Grinten, Tack, \& Hermus, 2010; Subbarayan, Dattani, Peters, \& Hindmarsh, 2014). Findings suggest that obesity in children with $\mathrm{CAH}$ is positively correlated with steroid dose. In patients with AI, therapy usually entails multiple daily dosing that can lead to high tissue exposure late in the afternoon and evening. Late afternoon glucocorticoid exposure has been linked to glucose intolerance, abdominal obesity, coronary atherosclerosis and fatigue/poor sleep pattern (Plat et al., 1999). More specifically, glucocorticoid exposure has been linked to an increase in visceral adipose tissue which is important because it is highly inflamed in individuals who are obese and as a result causes an inflammatory response that is associated with cardiovascular disease (Kim et al., 2016).

Moreover, it is also now understood that abdominal adiposity, and increase in BMI is positively correlated with an increased risk for metabolic syndrome and type 2 diabetes. The prevalence of diabetes and metabolic syndrome in older patients with CAH is underappreciated and may contribute to adverse HRQOL outcomes in the older population. A retrospective review of insurance claims data of $>10,000$ patients with AI examined the prevalence of comorbid conditions and hospital admissions. Results from this study suggest that diabetes mellitus may be of greater importance in patients with AI patients, and specifically the CAH population than previously recognized (Gunnarson et al., 2017).

Height. Final adult height has been an ongoing health issue in individuals with $\mathrm{CAH}$. The exact cause of short stature in $\mathrm{CAH}$ remains unclear; however, many believe that excessive androgens, early puberty, and under or overtreatment with glucocorticoids are contributing factors (Quintos, Vogiatzi, Harbison, \& New, 2000). Excessive androgen production during childhood often results in early linear growth, and early linear growth leads to premature epiphyseal maturation and closure (Lin-Su et al., 2008; New, 2006; Nimkarn et al., 2011). Therefore, individuals with CAH are usually tall as children, but have a final adult height that is 1.1 to 1.5 standard deviations below expected parental heights (Nimkarn et al., 2011). Eugester et al. (2001) conducted a retrospective chart review and meta-analysis to examine final height in individuals with $\mathrm{CAH}$. The results of the retrospective chart review were compared to the results of 18 studies in the metaanalysis. Children less than 5 years old were included in the retrospective chart review, and both children and adults were included in the 18 studies of the meta-analysis.

Eugester and colleagues concluded that final height was higher than previously reported values, but it was still lower than the overall population (Cordeiro et al., 2013; Eugster et al., 2001; Merke \& Poppas, 2013). Whether short stature itself constitutes a significant disability to children with CAH or is felt to adversely affect HRQOL is unknown. 
Reproductive Function, Infertility, and Pregnancy. Reproductive problems usually become obvious during adolescence (Speiser et al., 2010). Thus, excessive androgen production is associated with absent or irregular menses, absence of ovulation or an inadequate introitus in females with CAH (Demirci \& Witchel, 2008; Nimkarn et al., 2011). Additionally, menarche occurs late compared to their healthy counterparts when inadequately treated (White \& Speiser, 2000).

Fertility is linked to disease severity, psychosexual development, hormonal control and genital surgery (Jääskeläinen, Voutilainen, Hippeläinen, \& Kiekara, 2000). It is least common in non-classical $\mathrm{CAH}$, more frequent in simple virilizing $\mathrm{CAH}$, and most likely in salt-wasting CAH. Historical data shows that the rates of conception in females with classical $\mathrm{CAH}$ without fertility intervention are remarkably low with rates ranging from 0 to $10 \%$. However, such data may be misleading because it does not consider that many females with classical CAH never pursue pregnancy (Jääskeläinen et. al., 2000; Reichman, White, New, \& Rosenwaks, 2014). More recent data shows the fertility rate of individuals with $\mathrm{CAH}$ has increased and may be due to earlier management, improved compliance with treatment, and advanced surgical procedures (Mnif et al., 2013; Reisch, Arlt, \& Krone, 2011). Compared to reduced fertility, pregnancy is common and usually uneventful. Researchers examined the pregnancy rates of 81 females with salt-wasting CAH. While 9 of 81 females were pursuing pregnancy, 8 of the 9 were able to conceive (Casteras, De Silva, Rumsby, \& Conway, 2009; Reichman et al., 2014). Thus, it appears that fertility rates rather than pregnancy rates are reduced when compared to unaffected individuals (Casteras et al., 2009, Reisch et al., 2011).

Surgical Outcomes. Prenatal androgen exposure causes different degrees of virilization of the external genitalia in women, graded with Prader scores of $1-5$ (1=least virilized and $5=$ most virilized). More virilized females may have surgical correction advised at ages younger than 2 years. For patients with Prader stage greater than 3 , the Endocrine Society guidelines suggest that early surgery of the clitoris and vagina be performed in a single stage correction using the phallic tissue for vaginal reconstruction (Speiser et al., 2010). However, estrogen is felt to be positive for the healing process, thus, vaginal reconstruction approach after puberty has been proposed. There is not enough long-term evidence for one or the other and there are no studies comparing the psychological outcome of early vs. late surgery (Braga \& Pippi Salle, 2009). Females who have had early genital surgery experience several issues such as urinary incontinence, clitoral pain, vaginal stenosis as well as cosmetic concerns (Merke \& Poppas, 2013). The patient's own anatomy, the experience of the surgeon, the timing of the surgery, and the surgical technique itself will all influence the physical and psychological outcomes of the procedure itself.

\section{Quality of Life versus Health-Related Quality of Life}

Early conceptualizations of QOL were primarily broad and function-based, which emphasized physical functioning and general well-being (Cantrell \& Kelly, 2015). Years

later, patient-oriented outcomes - psychological and social domains of well-being-were 
added to this former concept of QOL. Hence, the term HRQOL was formed. Health related quality of life offers insight into individuals' perceived health and well-being. Often, HRQOL reflects the magnitude of dysfunction associated with an illness or medical treatment and is influenced by several circumstances that may affect how individuals perceive their health.

\section{Health-Related Quality of Life Research in Patients with CAH}

The rarity of $\mathrm{CAH}$ has made it challenging to conduct HRQOL studies in children. Therefore, much of what is known about the HRQOL in individuals with CAH has originated from adult research studies - many of which were conducted outside of the United States. Additionally, previous work on HRQOL of individuals with CAH and

similar conditions (such as disorders of sex development ([DSD]) has yielded conflicting results. Researchers have reported positive results or there was no difference in HRQOL when compared to the general population (Bachelot et al., 2007). In contrast, others have reported lower HRQOL scores in individuals with CAH and DSD, particularly in the social domains (Arlt et al., 2010; Mnif et al., 2012; Nermoen, Husebye, Svartberg, \& Lovas, 2010). A summary of studies evaluating the HRQOL of individuals with CAH are shown in Table 2-1.

\section{Qualitative Studies}

Nordenskjold et al. (2008) examined 62 Swedish women with CAH who ranged from 18 to 63 years. Each woman was matched with a control and both completed a 120 question semi-structured interview that addressed concerns related to surgery, surgical outcomes (i.e., function of clitoris and vagina), fertility, social life, and the health care they received during childhood. The results of the study indicated that the overall HRQOL is affected by many aspects of CAH such as type of mutation, type of surgical procedure, sexual relationships and fertility (Nordenskjold et al., 2008).

Malouf et al. (2010) studied the effects of CAH on HRQOL in a small group of North American women. The results from the interviews revealed 6 domains: physical functioning, psychological functioning, interpersonal processes and relationships, healthcare experiences of women with $\mathrm{CAH}$, recommendations for health care professionals, and systemic considerations. Many participants reported that they were functioning normally. However, normalcy is not explained by functioning alone, but social and emotional domains are also relevant to HRQOL. The results of this study imply that CAH influences all of the domains of HRQOL.

Schober et al. (2012) published a review paper which examined the HRQOL data in individuals with DSD from 1974 to 2012. They focused on surgical outcomes, QOL, quality of sex life, and medical and psychosocial risk factors. The review showed that QOL — specifically sex life — was impaired. Additionally, individuals with 46, XX DSD - 
Table 2-1. Summary of studies on HRQOL of patients with CAH and DSD

\begin{tabular}{|c|c|c|c|c|c|c|}
\hline Reference & Origin & $\begin{array}{l}\text { Study } \\
\text { Design }\end{array}$ & $\mathbf{N}$ & Diagnosis & $\begin{array}{l}\text { Measures to } \\
\text { Assess HRQOL }\end{array}$ & Main Findings \\
\hline Crawford et al., 2009 & Australia & $\begin{array}{l}\text { Cross- } \\
\text { sectional }\end{array}$ & 41 & $\begin{array}{l}\mathrm{DSD}(\mathrm{CAH}, \\
\mathrm{n}=16)\end{array}$ & PedsQL 4.0 & $\begin{array}{l}\text { Both children and parents } \\
\text { reported lower scores in } \\
\text { psychosocial domain when } \\
\text { compared to healthy children. }\end{array}$ \\
\hline Malouf et al., 2010 & USA & Qualitative & 8 & $\mathrm{CAH}$ & $\begin{array}{l}\text { Semi-structured } \\
\text { interview }\end{array}$ & $\begin{array}{l}\text { Identified } 6 \text { domains most } \\
\text { relevant to overall HRQOL of } \\
\text { women with CAH: physical } \\
\text { functioning, psychological } \\
\text { functioning, interpersonal } \\
\text { processes, relationships, } \\
\text { healthcare experiences, } \\
\text { recommendations for healthcare } \\
\text { professionals, systemic } \\
\text { considerations. }\end{array}$ \\
\hline Arlt et al., 2010 & UK & $\begin{array}{l}\text { Cross- } \\
\text { sectional }\end{array}$ & 203 & $\mathrm{CAH}$ & $\begin{array}{l}\text { SF-36, HADS, CAH } \\
\text { well-being (specific } \\
\text { non-validated) }\end{array}$ & $\begin{array}{l}\text { HRQOL was impaired in all } \\
\text { domains of the questionnaires. }\end{array}$ \\
\hline
\end{tabular}


Table 2-1. (Continued)

\begin{tabular}{|c|c|c|c|c|c|c|}
\hline Reference & Origin & $\begin{array}{l}\text { Study } \\
\text { Design }\end{array}$ & $\mathbf{N}$ & Diagnosis & $\begin{array}{l}\text { Measures to } \\
\text { Assess HRQOL }\end{array}$ & Main Findings \\
\hline Nermoen et al., 2010 & Norway & $\begin{array}{l}\text { Cross- } \\
\text { sectional }\end{array}$ & 72 & CAH & SF-36 and QOLS & $\begin{array}{l}\text { Scores of the overall QOLS } \\
\text { were the same as normal } \\
\text { population. All domains of the } \\
\text { SF-36 were impaired. }\end{array}$ \\
\hline Reisch et al., 2011 & Germany & $\begin{array}{l}\text { Cross- } \\
\text { sectional }\end{array}$ & 81 & CAH, PAI & $\begin{array}{l}\text { SF-36, GBB-24, } \\
\text { HADS }\end{array}$ & $\begin{array}{l}\text { No significant differences in } \\
\text { overall HRQOL of participants } \\
\text { with CAH and controls. } \\
\text { HRQOL was }\end{array}$ \\
\hline $\begin{array}{l}\text { Fagerholm et al., } \\
2012\end{array}$ & Germany & $\begin{array}{l}\text { Cross- } \\
\text { sectional }\end{array}$ & 24 & CAH, AIS & LSS 15D & $\begin{array}{l}\text { The majority of QOL and } \\
\text { HRQOL scores were within } \\
\text { normal range compared to the } \\
\text { control population. }\end{array}$ \\
\hline Schober et al., 2012 & France & Review & & DSD & & $\begin{array}{l}\text { Quality of life was impaired. } \\
\text { Individuals with CAH reported } \\
\text { lower sexual frequency, } \\
\text { avoidance, penetration } \\
\text { difficulty, asexuality, and global } \\
\text { sexual dysfunction. }\end{array}$ \\
\hline
\end{tabular}


Table 2-1. $\quad$ (Continued)

\begin{tabular}{|c|c|c|c|c|c|c|}
\hline Reference & Origin & $\begin{array}{l}\text { Study } \\
\text { Design }\end{array}$ & $\mathbf{N}$ & Diagnosis & $\begin{array}{l}\text { Measures to } \\
\text { Assess HRQOL }\end{array}$ & Main Findings \\
\hline Gilban et al., 2014 & Brazil & $\begin{array}{l}\text { Cross- } \\
\text { sectional }\end{array}$ & 25 & $\mathrm{CAH}$ & PedsQL 4.0 & $\begin{array}{l}\text { Overall HRQOL scores for } \\
\text { children and parents were lower } \\
\text { when compared to health } \\
\text { controls. Children's and parents } \\
\text { scores were also lower in the } \\
\text { physical and psychosocial } \\
\text { domains. }\end{array}$ \\
\hline $\begin{array}{l}\text { Jurgensen et al., } \\
2014\end{array}$ & Germany & $\begin{array}{l}\text { Cross- } \\
\text { sectional }\end{array}$ & $\begin{array}{c}84 \text { parents } \\
80 \\
\text { children }\end{array}$ & $\begin{array}{l}\text { DSD }(C A H, \\
n=33)\end{array}$ & KINDL-R & $\begin{array}{l}\text { Overall HRQOL was impaired } \\
\text { in children when compared to } \\
\text { unaffected children. Children } \\
\text { with DSDs reported lower } \\
\text { scores in the physical, self- } \\
\text { esteem, and school domains. } \\
\text { Parents reported lower scores in } \\
\text { the emotional well-being, but } \\
\text { found no impairment in the } \\
\text { child's overall HRQOL. }\end{array}$ \\
\hline Han et al., 2014 & UK & $\begin{array}{l}\text { Cross- } \\
\text { sectional }\end{array}$ & 101 & $\mathrm{CAH}$ & SF-36 & $\begin{array}{l}\text { Individuals with CAH have } \\
\text { worse HRQOL and all domains } \\
\text { of HRQOL are impaired. } \\
\text { Impaired HRQOL is a result of } \\
\text { treatment not the disease itself. }\end{array}$ \\
\hline
\end{tabular}


Table 2-1. (Continued)

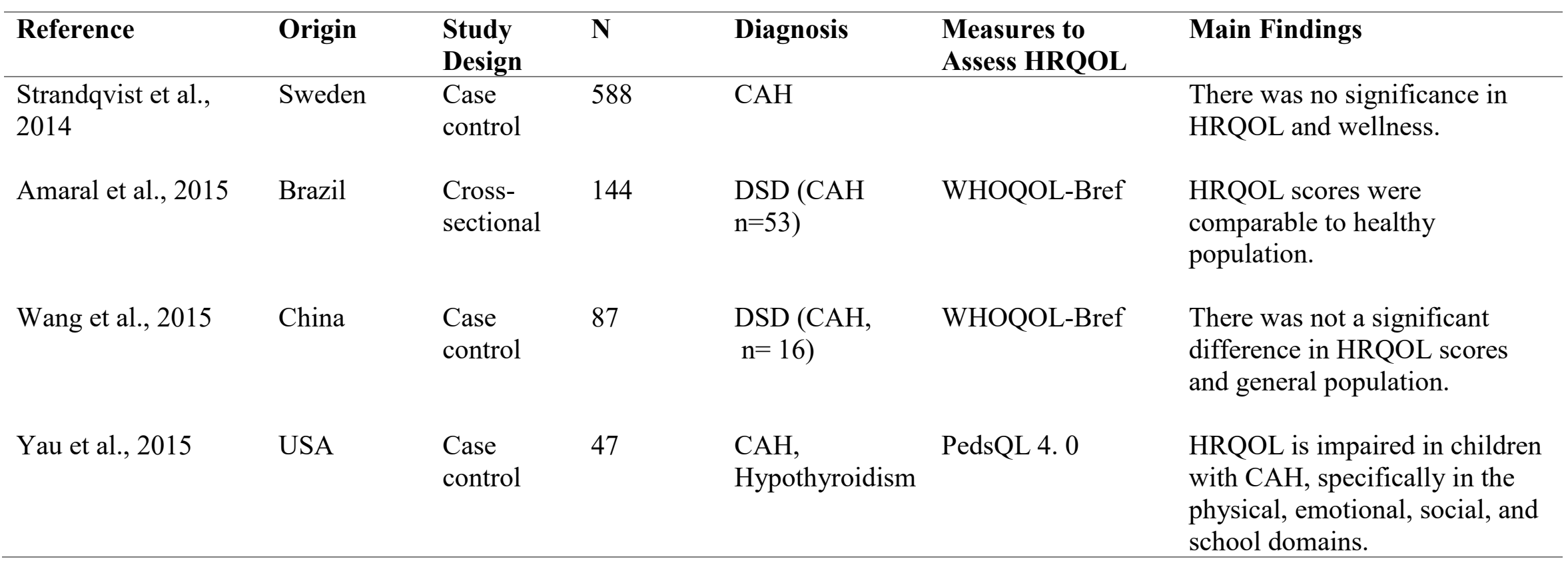

Note. $\mathrm{CAH}=$ congenital adrenal hyperplasia; DSD = disorder of sexual development; HRQOL = health-related quality of life; PedsQL = Measurement Model for the Pediatric Quality of Life Inventory; SF-36 = 36 -item Short Form Health Survey; HADS= Hospital Anxiety and Depression Scale; QOLS = Quality of Life Survey; PAI = Partial Androgen Insensivity; GBB-24 = Giessan Subjective Complaints List; AIS = Androgen insensitivity syndrome; LSS 15D = Life Situation Survey; QOL = quality of life; KINDL-R= KINDL-R Questionnaire for Measuring Health-Related Quality of Life in Children and Adolescents; WHOQOL-Bref = World Health Organization Quality of Life Survey. 
including individuals with $\mathrm{CAH}$ - reported lower sexual frequency, avoidance, penetration difficulty, asexuality, and global sexual dysfunction (Schober et al., 2012).

\section{Quantitative Studies}

A large British group examined the HRQOL of a cohort of 203 men and women with CAH. The SF-36, the 36 Item Short-Form Health Survey, and a CAH Wellbeing questionnaire were self-administered. SF-36 results showed impairments of HRQOL in all domains. With regards to the CAH Well-being questionnaire only $22 \%$ and $24 \%$ of female and male participants, respectively, reported concerns about the effect that CAH had on their long-term health (Arlt et al., 2010). In that same year, Nermoen et al. (2010) assessed 72 Norwegian men and women with classical CAH. The SF-36 and Quality of Life questionnaires were utilized. There was impairment in all domains of the SF-36, but the overall Quality of Life questionnaire scores were not lower when compared to the normal population. However, being physically fit, material comforts (i.e., home, food, financial security), and having a relationship with a spouse or significant other varied (Nermoen et al.).

In a cross-sectional study in Germany, researchers compared the HRQOL in participants with CAH with participants with partial androgen insensitivity (PAI) and healthy controls. The study utilized the SF-36, Giessan Subjective Complaints List (GBB-24), and Hospital Anxiety and Depression Scale (HADS). There were no significant differences in the overall HRQOL scores of CAH participants and controls. However, participants with CAH did report lower HRQOL in 3 domains of the GBB-24, but the SF-36 and HADS scores were no different than control group participants (Reisch et al., 2011b). Also, Fagerholm and colleagues evaluated the QOL and HRQOL of Finnish women with DSD. Sixteen of the 24 participants were diagnosed with CAH. The results of this study showed that $96 \%$ of the participants had HRQOL scores that were within normal range compared to the control population (Fagerholm, Taskinen, Mattila, Roine, \& Sintonen, 2012). Yet, a few patients reported concerns about delayed surgical treatment, poor surgical outcomes, and distressful memories.

Health-related quality of life data from a study conducted by Han et al. (2014) revealed that many individuals with CAH had worse overall HRQOL, and that all domains of HRQOL were impaired. Results from this study suggest that impaired HRQOL is a consequence of treatment, not the disease itself (Han et al., 2014). Furthermore, one of the largest epidemiological Swedish studies examined 335 women with CAH and reported no significant difference in HRQOL scores and wellness (Strandqvist et al., 2014). Yet, participants reported they were less likely to be married, had delays in psychosexual milestones, and a disinterest in sexual activity. Several others reported the negative impact of disease on their upbringing and the impact of disease on their sense of being different from the social environment. The study revealed that the quantitative measures did not reflect how the participants truly felt when analyzed qualitatively (Strandqvist et al.). 
Another study showed that HRQOL scores were comparable to a healthy Brazilian population (Amaral et al., 2015). Age at the beginning of treatment and timing of treatment were significantly associated with HRQOL scores. More specifically, chronological age at the beginning of treatment was negatively associated with HRQOL scores. Therefore, individuals who were treated early rather than later reported higher HRQOL scores. The most influential variables on HRQOL in participants were spirituality, religion and personal beliefs, positive feelings, and general health (Amaral et al., 2015). Wang and Tian (2015) also examined the HRQOL of individuals with DSDs living in China. Although the scores were lower in the psychological and environmental domains, the differences were not significant. There was a significant difference in the HRQOL of participants with DSDs and the general population (Wang \& Tian).

In 2009, Crawford and colleagues published one of the first research studies that exclusively evaluated HRQOL of children with DSDs. The sample consisted of 44 total participants of which 16 had a diagnosis of CAH. Both children and their parents completed quality of life questionnaires and were compared to a healthy sample. The scores for the physical QOL domain for both children and parents were the same as the healthy population. With regards to the psychosocial domain, female children and their parents reported lower QOL scores than their male counterparts and healthy children (Crawford, Warne, Grover, Southwell, \& Hutson, 2009).

Several years later, Jurgenseen, et al. (2014) examined the HRQOL in female children with DSD. The study consisted of 33 girls with CAH. In children 8 to 12 years old, overall HRQOL was impaired when compared to other children without DSD. More specifically, HRQOL was impaired in the self-esteem, physical well-being, and school domains. In comparison, parents reported lower scores in the emotional well-being domain but reported no impairment in the child's overall HRQOL (Jurgensen et al., 2014).

Subsequently, Gilban, Alves Junior, and Beserra (2014) and Yau, et al. (2015) also evaluated the HRQOL of children and adolescents with CAH from children's and parents' perspectives. Gilban et al. reported that self-assessment of HRQOL and proxyreported scores were similar in that they both indicated significantly lower scores for psychosocial, physical, and total scores when compared to healthy children. The results from Yau et al. showed that the overall HRQOL of children with CAH was impaired specifically in the physical, emotional, social, and school functioning domains when compared to healthy children. Parental perceptions were similar, and there was a strong correlation between child-self report and parent proxy-report.

It can be noted that different research designs were used in each of the aforementioned studies. Cross sectional and case control designs were predominant designs; whereas qualitative research designs were scarce. Also, the majority of studies were conducted in other countries and data were collected using self-report versus proxyreport measures. The ages ranged from 3 to 72 years old, and the number of participants varied. Some studies examined only children and females, while others included adults and both sexes. According to Zainuddin et al. (2013), this - different samples and 
methodologies - makes it challenging to compare results. The authors also note that the results of HRQOL and QOL are challenging because there are many conceptual components of HRQOL ranging from physical well-being to psychosocial adjustment and well-being (Zainuddin et al.).

\section{Bioecological Model of Human Development}

The bioecological model of human development (BMHD) focuses on development as an outcome over time. Within this model, development is conceptualized as the physical, cognitive, and social change of both and individual and group throughout the life course (Bronfenbrenner, 2004). The BMHD proposes that there are multiple stages and layers of development. Therefore, development is influenced not by a single construct, but by several constructs that accompany the individual throughout the life course. The theory identifies these constructs as: proximal processes, personal characteristics, contextual characteristics (environment) and time also known as the process-person-context-time (PPCT) model.

Similar to development, HRQOL is a multidimensional construct that describes the physical, mental, social, psychological, and functional well-being of an individual's perceived health (Bronfenbrenner, 2004; Ravens-Sieberer et al., 2001). Since HRQOL is a construct that encompasses several domains, an ecological perspective that includes multiple levels of analysis must be considered. Based on the ecological perspective, all aspects of health will affect several HRQOL domains in children and adolescents (Gaspar, Ribeiro, de Matos, Leal, \& Ferreira, 2012).

\section{Factors within the BMHD Model Identified in HRQOL Literature}

Process Characteristics. Relationships and social support - family relationships, peer relationships, and relationships with others - facilitate healthy or impaired HRQOL in individuals with CAH. Research suggests that the types of relationships and social support in childhood are reliable indicators of functioning later in life.

Malouf et al. (2010) affirms this. In a qualitative study of 8 women with $\mathrm{CAH}$, participants described their experiences with supportive/unsupportive people and behaviors, and how each affected their HRQOL. Some women discussed parental support and how their parents helped with medical management and "normalizing the condition" (Malouf et al., p .7). Other women talked about other important relationships, which included relationships with their siblings or friends. One participant noted that their friends' house was the best place to stay "... because they are more mature and they would have taken care of me if I needed it" (Malouf et al., p. 7). Other results from this study included unsupportive people and behaviors, which included the lack of sympathy from siblings and coworkers. This was reflected in the statement "It really hurt me to know my siblings resented me..." (Malouf et al., p. 7). With regards to relationships and 
social support, participants also noted having never met another individual with $\mathrm{CAH}$ (Gilban et al., 2014; Malouf et al., 2010).

In a similar study, women discussed the association between parent and peer relationships and their experience in living with $\mathrm{CAH}$. Women described their parents as "important sources of knowledge" (Engberg, Frisén, Möller, Hagenfeldt, \& Nordenskjöld, 2016, p. 24). One woman commented "my mother could talk doctor language so she was never afraid to ask...then she could explain to me" (Engberg et al., p. 24). Women also talked about the effects of parental feelings. Another woman expressed, "It felt like she (my mother) did not want anyone to know and that feeling is still with me today" (Engberg et al., p. 24). It became clear that parental relationships were important to the overall quality of life in women with $\mathrm{CAH}$.

Relationships with others are equally important as parent-child and peer relationships. Boyse, Gardner, Marvicsin, and Sandberg (2014) found that parents expressed a need for social support from other caregivers of children with CAH. Parents talked about how support from other caregivers is different and how this mediates the effects of CAH on HRQOL. One parent met another caregiver of a child with CAH and commented, "It was awesome. Just to share experiences....." (Boyse et al., p. 6). Another parent expressed feelings about the desire to reassure other parents. Results of this study indicate that parents, just like their children, value social support. Many found meaning in their child's diagnosis, which helped foster positive adjustment and HRQOL for the child and caregiver (Boyse et al.).

Person Characteristics. Person characteristics mediate or moderate the impact of CAH on HRQOL. Researchers propose that person characteristics are often reflected by individuals' attitude, approach to situations, or the outcome that is received which in turn impacts HRQOL (Wrosch \& Scheier, 2003). With regards to individuals with CAH, person characteristics that were associated with HRQOL were psychosexual differentiation (i.e., gender identity, gender role, sexual orientation) and psychological well-being.

Psychosexual Differentiation. Psychosexual differentiation includes three components: gender role, sexual orientation and gender identity. Gender role refers to behaviors and preferences-toys, play mates, style of play- that are typical of one gender (Pasterski et al., 2015). Sexual orientation refers to who an individual is most attracted. Gender identity refers to the sense of self as a male or female or neither. All three components of psychosexual differentiation have been associated with androgen exposure during fetal development. It has been shown that prenatal androgen exposure is dose dependent and can have a permanent effect on higher brain functions in utero (Frisen et al., 2009).

There is consistent evidence that girls with $\mathrm{CAH}$ are more likely to prefer male typical activities compared to unaffected females (Pasterski et al., 2015). Several studies report that females with $\mathrm{CAH}$ are more masculine, exhibit gender-atypical behavior, and are less interested in gender-typical activities as a child and adult (Cohen-Bendahan, van 
de Beek, \& Berenbaum, 2005; Hines, Brook, \& Conway, 2004; Nordenstrom, 2011). Reduced heterosexual orientation has also been reported several times in the literature. Although many women with $\mathrm{CAH}$ report a heterosexual orientation, there has been an increase of women reporting being sexually attracted to and having encounters with the same sex partners (Amaral et al., 2015; Frisen et al., 2009; Nordenstrom, 2011; Pasterski et al., 2015).

With regards to gender identity, there are discrepant findings. Pasterski et al. (2015) noted that 14 published studies examined gender identity in girls or women with CAH. Over half of the studies showed that women and girls with $\mathrm{CAH}$ have an increased masculine identity (Pasterski et al., 2015). In contrast, the remaining studies implied that gender identity in women and girls with $\mathrm{CAH}$ is no different from unaffected individuals. More recently, gender identity was examined in girls and boys with $\mathrm{CAH}$ from the perspective of the child and caregiver. When compared to controls, girls with CAH had more cross gender identification responses. Parents also indicated that girls with CAH display more cross gender behavior than unaffected girls (Pasterski et al., 2015).

Psychological Well-Being. Psychological well-being is fueled by experience and varies between individuals at different periods of time. In some studies, psychological well-being is associated with nondisclosure, stigma around having the condition, shame and secrecy (Cull, 2005). In others, it is associated with body image, and emotional, behavioral, and self-esteem problems.

Qualitative descriptions from women with DSD validate the association between psychological well-being and nondisclosure, secrecy, and stigma associated with the disease. Engberg et al. (2016) noted that women often considered CAH a private condition. Participants made comments such as "It is too personal, and it is not anyone's business", and "this disorder is so tough because you can't tell anyone about it because it is so strange and difficult and unpleasant" (Engberg et al., p. 24). Participants also commented on psychological support during childhood. Many recalled declining psychological support during childhood, but desired that same support later in life, "My biggest wish and hope is that these small children, girls, and teenagers will find it easier to confront this disorder because it is not like having an incurable disease; it is something much more intimate and private" (Engberg et al., p. 24). The authors also discuss the latent theme of shame, and how shame is internalized (i.e., negative self-evaluation) or externalized (i.e., other's perceptions). The results of this study emphasized the impact of psychological support and well-being during childhood, adolescence, and adulthood.

Furthermore, the most recent psychological well-being evaluation was investigated in a group of 110 Swedish adults with DSD. The study included 44 adults with CAH. Findings of this study revealed that individuals with DSDs exhibited high psychological distress which results in significantly impaired psychological well-being (Bennecke, Grüters, Köhler, Thyen, \& Lux, 2017). The researchers stressed the importance of care that not only focuses on physical well-being but also on psychological well-being (Bennecke et al., 2017). Other studies on psychological well-being are noted elsewhere (Sandberg, Robins, Litvak, \& Stout, 2010). 
For some children with $\mathrm{CAH}$, psychological well-being was linked to emotional and behavioral characteristics such as anxiety/depression, withdrawal, somatic complaints, aggressive behaviors as well as internalizing and externalizing behaviors (Berenbaum et al., 2004; Idris, Chandran, Syed Zakaria, \& Rasat, 2014). In a study that evaluated the emotional and behavioral problems of Indonesian children with DSDs, parents of children with DSDs reported more emotional problems than parents of children in the control group. Additionally, parents of children with $\mathrm{CAH}$ also reported that their daughters were more withdrawn from social activities (Ediati, et al., 2015). Idris et al. (2014) found contradictory results from previous studies. Researchers discovered that internalizing behavior problems were more prevalent in boys with $\mathrm{CAH}$ when compared to control groups. Boys reported having a greater number of anxious and aggressive behaviors. In contrast, psychosocial adjustment in girls fell within normal range, responses about aggression, anxiety/depression and higher energy levels fell within normal range when compared to control groups (Idris et al.).

Context Characteristics. Little consideration has been given to the impact of social contextual environments (i.e., religious, school, work, home) on the HRQOL of children and adults with CAH. Although not explicitly stated, contextual characteristics may lessen or worsen the effects of CAH on HRQOL. There is one study in which adults with $\mathrm{CAH}$ described their experiences and the impact of hospital visits. Here, participants noted that sometimes being admitted to the hospital was like "coming home" and was a "safe haven" (Engberg et al., 2016). Results of this study encourage the support of a medical home for children with CAH. A medical home supports the identification of patients' strengths, educational needs regarding the pathophysiology of CAH, cognitive abilities, mental health status, family support and need for surgery. So, the Congenital Adrenal Hyperplasia Research Education and Support (CARES) Foundation has provided a guide for medical homes, which is the ideal environment for children with $\mathrm{CAH}$ (Witchel, 2010).

Time. The element of time primarily focused on psychopathology of CAH (i.e., initial diagnosis/awareness of disorder, timing of surgery). Parents have reported that the period during initial diagnosis was confusing, confounding, shocking, filled with uncertainty and many attempted to make sense of the situation before and after diagnosis (Boyse et al., 2014; Lundberg, Roen, Lindstrom, \& Hegarty, 2017).

\section{Measuring Health-Related Quality of Life in Children with CAH}

Measuring HRQOL in children with disorders such as CAH comes with many challenges. These challenges include the child's developmental level, ability to understand their disease, what is being asked, and the use of caregivers as proxies (Connolly \& Johnson, 1999). Historically, investigators presumed that children's selfreports and caregiver proxy-reports should match, and proxy responses would be useful as supplemental information to what children report. However, researchers are realizing that caregivers as proxy responders also pose challenges (Bandayrel \& Johnston, 2014). For example, Eiser and Morse (2001b) discovered that caregivers are more able to 
evaluate observable aspects of health such as physical functioning than less observable aspects like self-esteem (Eiser \& Morse, 2001b). Evidence of this is supported by Jokovic and colleagues when they report that parents are more accurate in evaluating objective domains than domains that are internalized. They believe this to be true because children spend a considerable amount of time outside of the home and out of direct observation of the caregiver (Jokovic et al., 2004). Also, children continue to change, so there are some aspects that children and caregivers will not agree on.

Moreover, measuring HRQOL in children with $\mathrm{CAH}$ is also hindered by a lack of appropriate standardized instruments (Alpern, Gardner, Kogan, Sandberg, \& Quittner, 2017). Many are not sensitive to the unique experiences of children with CAH and DSD (Berenbaum et al., 2004; Sanberg \& Mazur, 2014; Schober et al., 2012). Likewise, most studies used generic HRQOL questionnaires, ad hoc scales that have not been validated, or used measures that primarily focused on surgical and psychosexual outcomes in adults (Alpern et al., 2017; Jürgensen, Kleinemeier, Kropf, Lux, \& Thyen, 2009; Pasterski et al., 2015; Sandberg et al., 2010). The lack of CAH specific assessment tools makes it challenging to inform and guide changes in practice and evaluate outcomes particularly for children (Alpern et al., 2017).

\section{Conclusion}

The results of this review suggest several fundamental issues remain to be addressed. A review of papers on CAH and HRQOL in developed and developing countries reveal a broad continuum of HRQOL outcomes ranging from very impaired, similar, to even better than control populations. Despite these inconsistences HRQOL results across studies must be interpreted with caution because of the different methodologies, sample size, age of participants, and access to patients and data. The literature also revealed several factors that contributed to HRQOL such as the results of over and under treatment, social, psychosexual, and psychological problems.

Furthermore, there were very few papers that evaluated children and adult perspectives of HRQOL, their experience of growing up with $\mathrm{CAH}$, and what HRQOL means to them. The lack of literature on individual perspectives results in a missed opportunity to better understand patients' lived experiences which is important because certain aspects of management of CAH have been controversial (Alpern et al., 2017). For example, former patients continue to voice concerns about the negative outcomes of surgery, which has ultimately influenced attitudes about when to perform surgery and the psychological consequences thereafter (Garland \& Diamond, 2014; Lantos, 2013). Additionally, there is a need to develop CAH and DSD specific HRQOL instruments for children and their caregivers as well as for adults. These measures would allow clinicians to identify specific needs of patients and families, evaluate the effects of medical and surgical treatment, and provide empirical evidence to guide clinical management (Alpern et al.). Addressing all of the aforementioned fundamental issues is important to improve the HRQOL of children and adults with CAH. 


\section{CHAPTER 3. METHODS}

This chapter includes a description of the research methods used in this study. The research design, sample, setting, protection of human subjects, instruments, procedures used during data collection, and data analyses are explained.

\section{Research Design}

A mixed methods design was used in this descriptive cross-sectional study to understand the HRQOL of female children with CAH, identify factors that contribute to HRQOL of female children with CAH, and compare measurements of HRQOL and factors that contribute HRQOL based on self-report with those from caregiver proxyreport. A convergent mixed methods design was selected whereby the quantitative (QUAN) and qualitative (QUAL) results were merged to provide a comprehensive understanding of HRQOL of female children with CAH that could otherwise not have been provided by either one of these methods alone (Morse \& Neihaus, 2009). In this study, QUAN and QUAL components were given equal emphasis. QUAN data were obtained from a demographic form and a validated questionnaire to measure overall as well as specific domains of HRQOL of female children with CAH. The QUAL component included semi-structured interviews and was conducted concurrently with the QUAN component. The QUAL component was used to explore children's and caregivers' perceptions of HRQOL to enhance the findings from the QUAN component.

\section{Sample and Setting}

The study was conducted in Memphis, TN between June 2016 and July 2017. However, study participants were recruited and accrued nationally (Figure 3-1). Twenty child-caregiver dyads participated in the QUAN and QUAL components and five childcaregiver dyads participated only in the QUANT component. The sample included children (age 7-18) who had been diagnosed with $\mathrm{CAH}$ and one of their caregivers.

\section{Protection of Human Subjects}

The University of Tennessee Health Science Center Institutional Review Board (UTHSC IRB) approved the study and related procedures used to recruit female children with $\mathrm{CAH}$ and their caregivers. Children are classified as vulnerable subjects because they have limited autonomy or limited ability to make deliberate and voluntary decisions and are more susceptible to harm (McGuire \& Chadwick, 2012). Participation in the study also included an adult family member with whom the child depends on to meet his/her needs (National Alliance for Caregiving, 2015). 


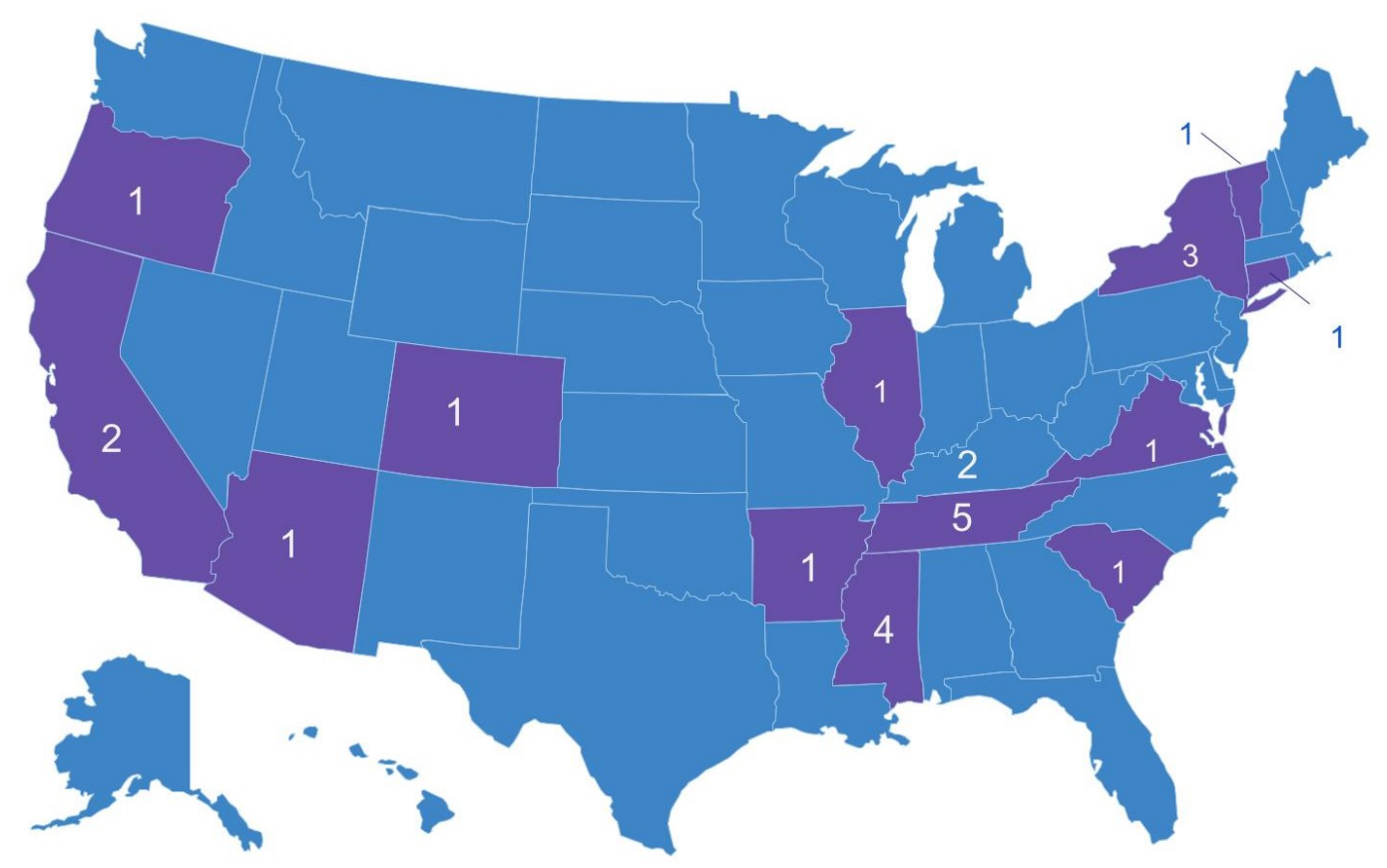

Figure 3-1. Map of participants by state 
The UTHSC IRB Standard Operating Procedures (SOP) for Informed Consent provides guidelines for obtaining assent from children 8 years or older who are believed to be capable of providing assent (UTHSC IRB, 2017). In this study, informed assent was provided prior to consent. For all child-caregiver dyads, informed consent was obtained from the caregiver (Appendix A).

The ethical principles of conducting research were included in all aspects of the study. The principle of justice was ensured by providing fair treatments to all study participants and maintaining participant privacy. Interview questions were designed to only elicit the necessary information needed to answer the research questions. Given the sensitive information being collected during the study, a certificate of confidentiality was obtained from the National Institute of Child and Human Development (Appendix B) to protect forced disclosure of sensitive, identifiable patient information should any research records be subject of legal subpoena (Polit \& Beck, 2008). Additional measures to ensure patient privacy involved data security. Each child-caregiver dyad was assigned an identification number which was used to identify participant data. A master list that contains participant names and ID numbers was created and was stored in a locked file cabinet. All other data collected were locked and stored on password protected computers and in locked file cabinets.

\section{Recruitment}

Flyers (Appendix C), participant interest letter (Appendix D) s, and participant referrals from the Le Bonheur Children's Hospital Endocrinology Clinic and the CARES Foundation were used to recruit this convenience sample. Flyers and participant interest letters with contact information were also displayed in Le Bonheur Children's Hospital Endocrinology Clinic and other community settings. Email messages through the CARES Foundation membership lists were also used to refer and enroll potential study participants. Flyers were also emailed to clinics around the U.S. that provide services to children with $\mathrm{CAH}$. A gift card to a national retail store in the amount of \$20.00 was offered to each child with $\mathrm{CAH}$ and to each caregiver that participated in the study. Recruitment goals included obtaining an adequate sample of female children with CAH and caregivers varying in demographic background to ensure generalizability of study findings.

\section{Participation Criteria}

Eligibility for the study was determined via telephone or through data extraction from medical records provided by Le Bonheur Children's Hospital. An authorized waiver of consent was obtained from the UTHSC IRB to extract personal information from medical records of participants who visit the Le Bonheur Children's Hospital Endocrinology Clinic.

Inclusion criteria for the study included the following: 
1. female child between the age of 7-18 years old at the time of enrollment

2. child has diagnosis of $\mathrm{CAH}$ due to 21-hydroxylase deficiency

3. caregiver over the age of 18 at the time of enrollment

4. the ability to read, understand, and speak English

5. the ability to understand the study and provide consent/assent to participate

The exclusion criteria for the study included the following:

1. female child over the age of 18

2. child has diagnosis of CAH not due to 21-hydroxylase deficiency

3. the inability to read, understand, and speak English

4. the inability to understand the study and provide consent/assent to participate

\section{Quantitative Instruments}

This study consisted of two quantitative instruments - a Demographic Form and KINDL-R Questionnaires. Both instruments captured data provided by the children and their caregiver and were used to quantify HRQOL and factors influencing HRQOL. In this study, the demographic data were used to identify factors that may contribute to HRQOL in female children with CAH. Additionally, the difference in self-report and caregiver proxy-report of HRQOL, and the associations between demographic health factors and HRQOL were examined.

Demographic Form. The Demographic Form was developed by the investigator and completed by the caregiver to obtain important information about the caregiver and the child with CAH. Questions petitioned information about caregiver age (in years), gender (female; male), ethnicity, marital status, educational (highest level of education completed), employment status, and geographic location (city; state). The form solicited information about the relationship of the caregiver to the child (parent; grandparent; sibling; other) and frequency of communication between the child and caregiver. The caregiver answered questions about the child's age, ethnicity, the grade in school, special services received at school, and insurance coverage. Caregivers were also questioned about the child's health status, other health conditions, and health care. Lastly, caregivers were asked to rate the health of the child with $\mathrm{CAH}$ on a rating scale from 1 to 10 whereby 1 indicated very poor health and 10 indicated very good health (Appendix E). Table 3-1 illustrates the demographic variables used in the study.

KINDL-R Questionnaire. The KINDL-R Questionnaire measures HRQOL in 317 year olds. The Kid-KINDL-R Children's Version (7-13- year- olds), the KiddoKINDL-R Teenager's Version (14 -17- year olds), and the KINDL-R Parent's Version were used in this study (see Appendices F-H). Each version of the questionnaire consists of 24 Likert-scaled items that measures 6 domains: Physical Well-Being (i.e., felt sick), 
Table 3-1. Sources of child and caregiver quantitative data

\begin{tabular}{|c|c|c|}
\hline \multirow[t]{2}{*}{ Variables } & \multicolumn{2}{|c|}{ Instrument by Respondent } \\
\hline & Child & Caregiver \\
\hline $\begin{array}{l}\text { HRQOL of children with CAH } \\
\text { Physical well-being } \\
\text { Emotional well-being } \\
\text { Self-esteem } \\
\text { Family } \\
\text { Friends } \\
\text { School } \\
\text { Total score }\end{array}$ & $\begin{array}{l}\text { Kid /Kiddo- } \\
\text { KINDL-R }\end{array}$ & KINDL-R Parent version \\
\hline HRQOL rating scale score & & Demographic form \\
\hline $\begin{array}{l}\text { Demographics for children with CAH } \\
\text { Age } \\
\text { Diagnosis } \\
\text { Ethnicity } \\
\text { Extracurricular activities } \\
\text { Frequency of medication } \\
\text { (hydrocortisone) } \\
\text { Frequency of stress dose } \\
\text { Frequency of ER Visits } \\
\text { Other Health Issues } \\
\text { Health Insurance }\end{array}$ & & Demographic form \\
\hline $\begin{array}{l}\text { Demographics for caregivers } \\
\text { Age } \\
\text { Race } \\
\text { Gender } \\
\text { Marital status } \\
\text { Education } \\
\text { Employment status } \\
\text { Frequency of communication about } \\
\text { CAH }\end{array}$ & & Demographic form \\
\hline
\end{tabular}


Emotional Well-Being (i.e., felt insecure), Self-Esteem (i.e., was happy with myself), Family (i.e., felt comfortable at home), Friends (i.e., got along well with others), and Everyday Functioning or School (i.e., was afraid of getting bad grades) (Ravens-Sieberer $\&$ Bullinger, 2000). These domains are represented by six subscales. Children were asked to respond to each item according to how things have been for them during the past week. Caregivers were asked to respond to each item according to how their child had been feeling during the past week. The following responses are provided for each item: never, seldom, sometimes, often, and all the time, with scores ranging from 1 (never) to 5 (all the time). The item responses are reverse coded with values from 1 to 5 with higher scores indicating a higher HRQOL. Scores on ten items $(1,2,3,6,7,8,15,16,20$, and 24) were reverse scored. In the KINDL-R Parent's Version and the Kiddo- KINDL-R Teenager's Version, item 23 was also reversed. Following reversal of scores, the item scores in each domain were totaled to yield a subscale score. That is, the Physical Wellbeing subscale score is the sum of scores on items 1, 2, 3, and 4 divided by 4 (number of subscale items). Emotional Well-being subscale score is the sum of scores on items 5, 6, 7 , and 8 divided by 4 (number of subscale items). All subscale scores were calculated using this method. The total HRQOL score was calculated by combining the subscale scores. The item scores for each subscale and the total HRQOL scores were then added and transformed to scores ranging from 0 to 100 . The total HRQOL scores of the KINDL-R quantitatively represent children's perception of HRQOL or parent's perceptions of their child's HRQOL.

Psychometric properties of the KINDL-R were calculated from a German sample of 7,166 children and their caregivers (Erhart, Ellert, Kurth, \& Ravens-Sieberer, 2009). To measure the reliability of the KINDL-R the Cronbach's alpha was calculated, and values above 0.70 for both total scores and subscale scores were considered acceptable. Within the self-report version, the Cronbach's alpha values for total scores and subscale scores were lower than the proxy-report versions. For the self-report questionnaires, the Cronbach's alpha was 0.83 and values ranged from 0.53 to 0.72 for the subscales. For the proxy version, the Cronbach's alpha was 0.86 for the total score and values ranged from 0.62 to 0.74 for the subscale scores. Both versions detected lower internal consistency in younger children compared to adolescents (Erhart et al.).

Linear structural equation modeling was used to assess the validity of the KINDLR. The LISREL 8 Software was used to conduct a confirmatory factor analysis. Root Mean Square Residual (RMSEA) were utilized to validate the goodness of fit of the models and the data. Hierarchical sequence confirmatory factory analysis models were conducted and showed an acceptable fit for the 6 subscales of the parent (RMSEA $=0.07)$ and child (RMSEA=0.06) versions. Independent goodness-of-fit evaluations for the selfreport and the parent-report versions showed similar results (self-report: RMSEA $=0.064$, $\mathrm{AGFI}=0.944$; parent report: $\mathrm{RMSEA}=0.069, \mathrm{AGFI}=0.965)($ Erhart et al., 2009, p. 5).

Convergent validity was measured by calculating the Pearson's correlation coefficient between the two KINDL-R versions and the Strengths and Difficulties (SDQ) self and parent versions. It was expected that the KINDL-R Psychological well-being dimension would reveal the strongest correlation with the SDQ Emotional Scale. The 
subscale of Family was expected to correlate to the SDQ Conduct Scale. The KINDL subscale of Friends was expected with the SDQ Peer Problems scale. Lastly, the total HRQOL score of the KINDL-R would most closely be correlated with SDQ Total difficulties score (Erhart et al., 2009).

The KINDL subscales of Psychological (Emotional) Well-Being, Family, and Friends exhibited convergent validity with SDQ scales; coefficients ranged between 0.33 and 0.49. The KINDL-R self-report total score was highly correlated with the SDQ selfreport Total difficulties score $(\mathrm{r}=0.57)$. Discriminant validity was displayed by lower correlations of the KINDL-R subscales and other SDQ scales. Also, the KINDL-R selfreport displayed convergent and discriminant validity with the SDQ parent-report version. The KINDL-R Psychological Well-Being did not display convergent validity with the reported SDQ Emotional scale $(r=0.30)$ (Erhart et al., 2009).

The KINDL-R Parent version displayed convergent validity with the SDQ parent version. The KINDL-R total score of the parent version was highly correlated with the parent version SDQ Total difficulties score $(r=0.63)$. The KINDL-R parent version total score showed convergent and discriminant validity with the self-report SDQ Total difficulties score $(\mathrm{r}=0.33)$, and the Friend subscale score showed convergent and discriminant validity with the SDQ Peer problems $(\mathrm{r}=0.32)$. There were similar patterns of correlation between the KINDL-R and SDQ across age groups. Psychometric results indicate that both self-report and proxy-report versions of the KINDL-R are reliable in assessing the HRQOL (Erhart et al., 2009).

\section{Qualitative Instrument}

Data regarding perceptions of HRQOL and factors that may influence HRQOL were also obtained through semi-structured interviews conducted with children and their caregiver. The interview guide consisted of 10 broad questions with a subset of questions for each broad category to encourage sharing information in greater details and discourage acquiescence. The first three questions solicited information about communication and disclosure of diagnosis to others such as family members, friends, and other people in the caregiver and child's life. Caregivers were asked about how they talk about $\mathrm{CAH}$ with the child, and children were asked how they talked about CAH with their caregiver. The next two questions transitioned into children's and caregivers' perception of the child's health, and the child's experience living with CAH. There was one question that addressed peer relationships and the child's ability to get along with others. The following two questions related to the child's self-esteem and sense of belonging. The next question focused on what was most important to the child and how to improve the lives of children with $\mathrm{CAH}$. The last question summarized what was heard in the interview and asked the participant to validate, confirm, correct, and add to what was heard by the interviewer (see Appendices I-J). 


\section{Sources of Data}

The variables measured in this study are presented in Table 3-1. The respondent who provided the data for each variable is indicated.

\section{Procedure}

The study's purpose and procedures were explained to participants, questions about the study were answered, and assent/consent was obtained during face-to-face private meetings with the child-caregiver dyad. After assent/consent was obtained, the child and her caregiver were asked to sit separately, in a location to secure privacy and confidentiality of answers. The caregiver was asked to complete the Demographic Form. The investigator remained with the child and began the semi-structured interview using the Interview Guide for Child/Adolescent.

The interview with each participant lasted approximately 30 to 90 minutes. The interview was audio recorded, and the audio recorder was visible to the investigator, the child, and caregiver. The investigator asked the child open-ended questions with the goal of soliciting personal perspectives. The questions were asked using developmentally appropriate language for children and adolescents.

The KINDL-R was then administered to the child with CAH. During data collection, the investigator observed the child and offered assistance or clarification with responding to specific items as needed. After the child completed study procedures, the child was asked to remain separate from the caregiver. The caregiver was also interviewed, and asked to complete the KINDL-R after the interview.

For participants who were unavailable for face-to face meetings, assent and consent were obtained via telephone. Informed consent forms and surveys were mailed along with addressed envelopes with pre-postage forms to be returned to the investigator. After the consent forms were returned, the interviews were scheduled and conducted over the telephone with the child and caregiver. During the phone interview, the child and her caregiver were asked to sit separately, in a location to secure privacy and confidentiality of responses. After the separate interviews were completed with the child and with the caregiver, each was asked to continue to sit separately and complete the KINDL-R. The interviews were audio recorded and recorded data were stored on a password protected computer.

For child-caregiver dyads that only wanted to complete the KINDL-R, a web link that directed participants to a web-based survey tool was provided. The first page of the web-based survey contained a survey consent form that provided details about the survey portion of the study. The online consent form also included the purpose, procedures, risks associated with participation, payment for participation, contact information for questions or concerns, and the option to accept or decline participation (Appendix K). Once study 
procedures were complete, the child and her caregiver each received a $\$ 20.00$ gift card to a retail store.

\section{Data Analysis}

Quantitative data were entered into the International Business Machines Corporation Statistical Package for the Social Sciences (SPSS) version 24 for analysis. Descriptive statistics (frequency, percentage, median, and interquartile range) were calculated for categorical and continuous variables where appropriate. The Wilcoxon signed-rank test and Fisher's exact test were used to test median of paired differences between child and caregiver HRQOL scores and associations, respectively. Fisher's exact test was used to calculate all analyses due to small expected cell counts. All p-values $<$ 0.05 were considered statistically significant.

Descriptive content analysis using a hermeneutical, phenomenological framework was applied to describe children's and their caregiver's perceptions of HRQOL and lived experiences by theme (van Manen, 1990; Earle, 2010). Hermeneutic phenomenology focuses on highlighting the details and trivial aspects of individuals' lived experience that may normally be taken for granted. Furthermore, it seeks to understand the meaning of individuals' lived experiences. This framework was selected because it is compatible with the bioecological model in that it takes into consideration the uniqueness of the person and the subjective experiences of individuals. Additionally, the framework utilizes the person as a source of knowledge thus allowing researchers to find meaning in human interaction allowing for greater insights into developing individuals' perceptions about HRQOL.

All interviews were transcribed and entered into NVivo 10 software (QSR International Pty Ltd) for analysis. Data analysis consisted of line-by-line coding of the interview data. The codes were then grouped into categories, and further grouped into overarching categories and themes.

Trustworthiness was explored through credibility and reliability to demonstrate integrity of the qualitative findings (Creswell, 2007; Lincoln \& Guba, 1985). A homogenous group in terms of diagnosis, gender, and age gave credibility to the sample. The descriptions of the child and caregiver experiences were transcribed verbatim and checked for accuracy. Respondent validation procedures were carried out for selected caregiver-child dyads. To accomplish this, a narrative summary was created for select dyads. The summary was presented to the child and caregiver separately to cross check the investigator's analysis and interpretation of the interview responses.

\section{Specific Aim One}

The first specific aim was to measure the HRQOL of female children with CAH. The following research question pertains to the first specific aim: 
Research Question 1: What is the HRQOL of female children with CAH as reported by:
a. children with $\mathrm{CAH}$ ?
b. caregivers of children with $\mathrm{CAH}$ ?

Health-related quality of life was measured using the KINDL-R questionnaire self-report version and parent version. Descriptive statistics were used to describe responses from children with $\mathrm{CAH}$ and their caregiver.

\section{Specific Aim Two}

The second specific aim was to examine the differences and associations between child self-report and caregiver proxy report of the HRQOL of female children with $\mathrm{CAH}$.

Research Question 2: Are there any differences between child self-report and caregiver proxy-report KINDL-R scores?

Research Question 3: Are there any associations between demographic health factors and HRQOL graphic rating scores as reported by caregivers of female children with $\mathrm{CAH}$ ?

The KINDL-R self-report responses were compared to the caregiver proxy-report responses using a non-parametric test, specifically the Wilcoxon signed-rank tests.

Pearson's chi square tests were used to explore associations between demographic health factors and the scores from the HRQOL graphic rating scale.

\section{Specific Aim Three}

The third aim was to explore the HRQOL and factors that contribute to HRQOL of female children with $\mathrm{CAH}$ from the perspectives of children with $\mathrm{CAH}$ and their caregiver.

Research Question 4: What themes on HRQOL and factors that may contribute to the HRQOL of children with CAH emerged during interviews among:

a. children with CAH?

b. caregivers of children with $\mathrm{CAH}$ ?

Semi-structured interview data were analyzed using a hermeneutical, phenomenological framework to identify themes that describe children's and caregivers' perceptions of HRQOL and factors that may contribute to the HRQOL of children with $\mathrm{CAH}$. 


\section{Specific Aim Four}

The last aim was to compare the quantitative and qualitative child and caregiver results on HRQOL of female children with $\mathrm{CAH}$.

Research Question 5: To what extent do the themes on HRQOL emerging from interviews with children support the self-reported HRQOL as measured by the KINDL-R?

Research Question 6: To what extent do the themes on HRQOL emerging from interviews with caregivers support the caregiver proxy-reported HRQOL as measured by the KINDL-R?

Research Question 7: To what extent do the themes on HRQOL emerging from interviews with children and their caregivers support the self-report and caregiver proxy-reported HRQOL as measured by the KINDL-R?

Qualitative interview responses from the child were compared to the quantitative data of the KINDL-R. Qualitative interview responses from the caregiver were compared to the quantitative caregiver proxy responses of the KINDL-R. Qualitative interview data from the child-caregiver dyad were compared to the quantitative responses of both the self-report and caregiver proxy report responses of the KINDL-R. The data were analyzed individually and merged to corroborate findings. 


\section{CHAPTER 4. RESULTS}

The purpose of this study was to understand the HRQOL of female children with $\mathrm{CAH}$ and factors that may affect the HRQOL of female children with $\mathrm{CAH}$ from the perspective of the child and caregiver. This chapter presents the quantitative and qualitative results of the analysis.

\section{Description of the Sample}

The characteristics of the 25 female children with CAH and their caregivers are presented in Tables 4-1 and 4-2. The median age of children was 11 years with a range of 7 to 18 and interquartile range (IQR) 9- 14 years. Over half $(60 \%)$ of the children had a diagnosis of classical $\mathrm{CAH}$; the remaining (40\%) had non-classical $\mathrm{CAH}$. The majority of children were White (72\%), followed by Black or African American (12\%) and other $(12 \%)$. One third of the sample $(8 / 25)$ had other issues such as anxiety, hypothyroidism, vision problems, attention deficit disorder, hearing loss, abdominal migraines, and hypertension. The majority of children were involved in extracurricular activities. The frequency of daily medication (taking hydrocortisone) varied from once a day to not at all. Several children had to use stress dose of steroids at least twice a year. Many of the children had never visited the emergency room (ER) or visited the ER less than once a year (i.e. once during childhood).

Caregiver's median age was 45 years with range of 29 to 62 and an interquartile range (IQR) of 39-51. There were 23 female caregivers and 2 male caregivers of children with CAH. The majority of caregivers were White (76\%), followed by Black or African American (12\%). Most caregivers had completed at least 2 years of college. Thirty-two percent had at least 2 years of college or Associate's degree; sixteen percent had a Bachelor's Degree, twenty-eight percent had a Graduate or Master's Degree. The highest level of education completed by the caregiver was a doctoral degree (12\%).

\section{Specific Aim One}

The first specific aim was to measure the HRQOL of female children with CAH. The following research question pertains to the first specific aim:

Research Question 1: What is the HRQOL of female children with CAH as reported by:

a. children with $\mathrm{CAH}$ ?

b. caregivers of children with $\mathrm{CAH}$ ?

Health-related quality of life was measured using the KINDL-R questionnaire which was administered verbally, in written form, and online. The data were entered, 
Table 4-1. Demographics and health characteristics of children with CAH

\begin{tabular}{|c|c|}
\hline Characteristics & $\mathbf{N}=\mathbf{2 5}$ \\
\hline Age (in years), Median (IQR) & $11(9-14)$ \\
\hline \multicolumn{2}{|l|}{ Race, n (\%) } \\
\hline White & $18(72)$ \\
\hline Black or African American & $3(12)$ \\
\hline American Indian or Alaskan Native & $1(4)$ \\
\hline Other $^{\mathrm{a}}$ & $3(12)$ \\
\hline \multicolumn{2}{|l|}{ Diagnosis, $\mathrm{n}(\%)$} \\
\hline Classical & $15(60)$ \\
\hline Non-classical & $10(40)$ \\
\hline \multicolumn{2}{|l|}{ Other health issues ${ }^{\mathrm{b}}, \mathrm{n}(\%)$} \\
\hline Child has other health issues & $8(32)$ \\
\hline Child does not have other health issues & $17(68)$ \\
\hline \multicolumn{2}{|l|}{ Health Insurance, n (\%) } \\
\hline Private & $18(72)$ \\
\hline Medicaid & $7(28)$ \\
\hline \multicolumn{2}{|l|}{ Extracurricular Activities, n (\%) } \\
\hline Participates in extracurricular activities & $21(84)$ \\
\hline Does not participate in extracurricular activities & $4(16)$ \\
\hline \multicolumn{2}{|l|}{ Frequency of Medication (hydrocortisone), $\mathrm{n}(\%)$} \\
\hline Once a day & $2(8)$ \\
\hline Twice a day & $5(20)$ \\
\hline Three times a day & $9(36)$ \\
\hline Other ${ }^{\mathrm{c}}$ & $9(36)$ \\
\hline \multicolumn{2}{|l|}{ Frequency of Stress Dose, n (\%) } \\
\hline Twice a month & $1(4)$ \\
\hline Monthly & $1(4)$ \\
\hline Twice a year & $7(28)$ \\
\hline Once a year & $2(8)$ \\
\hline Otherd & $14(56)$ \\
\hline
\end{tabular}


Table 4-1. (Continued)

\begin{tabular}{lc}
\hline Characteristics & $\mathbf{N}=\mathbf{2 5}$ \\
\hline Frequency of ER Visits, $\mathrm{n}(\%)$ & $1(4)$ \\
Twice a month & $2(8)$ \\
Twice a year & $6(24)$ \\
Once a year & $16(64)$ \\
Other & \\
\hline
\end{tabular}

Note: The sample reflects the full sample for the qualitative and quantitative portion of the study. IQR = interquartile range.

${ }^{\text {a }}$ Participants identified themselves as having more than one race.

${ }^{\mathrm{b}}$ Other health issues includes anxiety, hypothyroidism, vision problems, attention deficit disorder, hearing loss, abdominal migraines and hypertension.

${ }^{\mathrm{c}}$ Participants steroid dosing regimen varied from never/no longer taking steroids, or they were taking a different type of steroid such as dexamethasone.

${ }^{\mathrm{d}}$ Stress dose is when the child has to take extra steroids because the body is stressed. In this study, participants stressed dose less than once a year, as needed or had never stressed dose.

${ }^{\mathrm{e}}$ Participants visited the emergency room (ER) less than once a year (i.e., once during childhood) or had never visited the ER. 
Table 4-2. Demographic characteristics of the caregivers

\begin{tabular}{lc}
\hline Characteristics & $\mathbf{N}=\mathbf{2 5}$ \\
\hline Age (in years), Median (IQR) & $45(39-51)$ \\
Gender, n (\%) & \\
Female & $23(92)$ \\
Male & $2(8)$ \\
Race, $\mathrm{n}(\%)$ & \\
White & $19(76)$ \\
Black or African American & $3(12)$ \\
American Indian or Alaskan Native & $1(4)$ \\
Asian & $1(4)$ \\
Other & $1(4)$ \\
& \\
Marital Status, n (\%) & \\
Married & $17(68)$ \\
Divorced & $4(16)$ \\
Single & $3(12)$ \\
Widowed & $1(4)$ \\
Education, $n$ (\%) & \\
High School Diploma or GED & $3(12)$ \\
Some College/Associate's Degree & $8(32)$ \\
Bachelor's Degree & $4(16)$ \\
Graduate/Master's Degree & $7(28)$ \\
Doctoral Degree & $3(12)$ \\
Employment Status, n (\%) & \\
Full Time (30 hours or more per week) & \\
Part Time (29 hours or less per week) & $15(60)$ \\
Stay at home caregiver & $5(20)$ \\
Retired & $3(12)$ \\
Frequency of communication on CAH, n (\%) & $2(8)$ \\
Daily & \\
Three or four times a week & \\
Once or twice a week & $4(16)$ \\
Three or four times a year & $3(12)$ \\
Twice a year & $6(24)$ \\
Monthly & $4(16)$ \\
& $1(4)$ \\
& $7(28)$ \\
\hline
\end{tabular}


Table 4-2. (Continued)

Note: The sample reflects the full study sample for the qualitative and quantitative portion of the study. IQR= interquartile range.

${ }^{\text {a }}$ Participants identified themselves as having more than one race. 
scored, and aggregated using the International Business Machine Corporation Statistical Package for Social Sciences (SPSS) version 24. For all scale scores, higher values indicate a higher HRQOL.

\section{Research Question 1 Findings}

The median $(M d n)$ and IQR of the child self-report and caregiver proxy-report of HRQOL are provided in Table 4-3. Per self-report the median total HRQOL score was $(M d n) 69.8(\mathrm{IQR}=62.5-79.2)$ Younger children $(M d n=19,7-13$ years $)$ reported higher HQOL scores compared to adolescents $(M d n=6,14-18$ years). The lowest total HRQOL score reported by children was 46.88 and the highest score was 83.33. Children scored highest on the Emotional Well-Being and Friends subscale which is comparable to the reference population. However, self-reported Self-Esteem and School sub-scale were the lowest scores and below the national average for the reference population (RavensSieberer \& Bullinger, 2000; Ravens-Sieberer, Erhart, Wille, Bullinger, \& the BELLA Study Group, 2008).

The median caregiver proxy-reported total HRQOL score was $71.9(\mathrm{IQR}=64.1$ 77.1). The lowest total HRQOL score reported by caregivers was 42.71, and the highest score was 86.46. Similar to children, the highest median scores for caregivers were on the Emotional Well-Being $(M d n=81.3$ 71.9-87.5) and Friends $(M d n=75,68.8-87.5)$ subscales which were comparable to the normative reference population of caregivers.

\section{Specific Aim Two}

The second specific aim was to examine the differences and associations between child self-report and caregiver proxy report of the HRQOL of female children with CAH?

Research Question 2: Are there any differences between child self-report and caregiver proxy report KINDL-R scores?

\section{Research Question 2 Findings}

Median and interquartile range (IQR) used in specific aim one was used to compare the HRQOL scores of children obtained by self-report and caregiver-proxy report. Paired differences between the HRQOL scores and subscale scores of children and caregivers are presented in Table 4-3. There was a statistically significant difference in the School subscale of the KINDL-R $(M d n=12.5, \mathrm{IQR}=0-18.75)$. The lowest School subscale score reported by children was 18.75 and the highest score was 100 . The lowest School subscale score reported by caregivers was 25 and the highest score was 93.8. In this study, the child and caregiver who reported the lowest School subscale scores also reported that the child had other health issues such as hypothyroidism and anxiety. There 
Table 4-3. KINDL-R health related quality of life scores of female children with CAH obtained via self-report $(n=25)$ and caregiver proxy-report $(n=25)$

\begin{tabular}{lcccc}
\hline KINDL-R & $\begin{array}{c}\text { Child } \\
\text { Median (IQR) }\end{array}$ & $\begin{array}{c}\text { Caregiver } \\
\text { Median (IQR) }\end{array}$ & $\begin{array}{c}\text { Paired } \\
\text { Difference } \\
\text { Median (IQR) }\end{array}$ & p Value \\
\hline $\begin{array}{l}\text { Physical Well- } \\
\text { Being }\end{array}$ & $68.8(56.3-81.3)$ & $68.8(43.8-78.1)$ & $-6.25(-12.5-0)$ & .208 \\
$\begin{array}{l}\text { Emotional Well- } \\
\text { Being }\end{array}$ & $81.3(62.5-87.5)$ & $81.3(71.9-87.5)$ & $6.25(-6.25-12.5)$ & .415 \\
Self-Esteem & $62.5(53.1-75.0)$ & $68.8(59.4-78.1)$ & $0(-6.25-12.5)$ & .387 \\
Family & $68.8(62.5-87.5)$ & $68.8(59.4-75)$ & $-6.25(-18.75-6.25)$ & .272 \\
Friends & $81.3(68.8-90.6)$ & $75.0(68.8-87.5)$ & $-6.25(-12.5-6.25)$ & .232 \\
School & $56.3(43.8-71.9)$ & $68.8(56.3-81.3)$ & $12.5(0-18.75)$ & $.005^{*}$ \\
Total & $69.8(62.5-79.2)$ & $71.9(64.1-77.1)$ & $-2.09(-4.17-6.2)$ & .786 \\
\hline
\end{tabular}

Note. Wilcoxon signed-rank test used to compute differences between child and caregiver groups. IQR indicates interquartile range.

$*$ Paired difference is statistically significant at $\mathrm{p}<0.05$. 
was no significant difference in the other subscale scores and the total scores between the children's and caregivers' responses.

Research Question 3: Are there any associations between demographic health factors and HRQOL graphic rating scores as reported by caregivers of children with CAH?

\section{Research Question 3 Findings}

Fisher's exact tests were conducted to examine relationships between demographic health characteristics and the HRQOL rating scale, health characteristics and diagnosis, and health characteristics and frequency of communication. The results are provided in Table 4-4, Table 4-5, and Table 4-6. There was a significant relationship with how caregivers rated children's HRQOL and if children had other health issues, $\mathrm{p}=$ .024. In this study, 22 (88\% of total) caregivers rated their child's HRQOL as 7 or above which indicates a good HRQOL. Of those, 17 (77.3\%) did not have any other health issues. The remaining three caregivers who reported children had significant health issues such as anxiety and high blood pressure all rated their child's HRQOL as fair. More specifically, those caregivers rated their child's HRQOL as 4 and 6. Thus, it appears that the caregivers believe that other health issues affect children's HRQOL and they are more likely to rate children's HRQOL as good.

Of the caregivers that rated children's HRQOL less than good, $2(67 \%)$ had classical CAH and $1(33.3 \%)$ had non-classical CAH. Six (40\%) of 15 children with classical CAH took hydrocortisone three times a day compared to $3(30 \%)$ children with non-classical CAH.

Many caregivers and children in this study had weekly (9 or $36 \%$ of total) or monthly ( 7 or $28 \%$ of total) discussions about CAH. Of those who had monthly discussions about $\mathrm{CAH}, 6(85.7 \%)$ had stress dosed less than once a year or never, and visited the emergency room (ER) less than once a year or never (6 or $85.7 \%$ ).

\section{Specific Aim Three}

The third aim was to explore the HRQOL and factors that contribute to HRQOL of female children with $\mathrm{CAH}$ from the perspectives of children with $\mathrm{CAH}$ and their caregiver.

Research Question 4: What themes on HRQOL and factors that may contribute to the HRQOL of children with CAH emerged during interviews with:

a. children with CAH?

b. caregivers of children with $\mathrm{CAH}$ ? 
Table 4-4. Associations between demographic health characteristics and HRQOL rating scale

\begin{tabular}{lccc}
\hline \multirow{2}{*}{$\begin{array}{l}\text { Demographic Health } \\
\text { Characteristic }\end{array}$} & \multicolumn{2}{c}{ HRQOL } & \\
\cline { 2 - 3 } Diagnosis & $2(67 \%)$ & $13(59.1 \%)$ & 1.0 \\
Classical CAH & $1(33 \%)$ & $9(40.9 \%)$ & \\
Non-classical CAH & & & \\
& $3(100 \%)$ & $5(27.7 \%)$ & $.024^{*}$ \\
Other health issues & $0(0 \%)$ & $17(77.3 \%)$ & \\
Yes & & & \\
No & & & \\
& & & \\
Frequency of stress dose & $0(0 \%)$ & $2(9.1 \%)$ & 1.0 \\
Twice a month & $1(33.3)$ & $6(27.3 \%)$ & \\
Twice a year & $0(0 \%)$ & $2(9.1)$ & \\
Once a year & $2(67.7)$ & $12(54.5 \%)$ & \\
Other & & & \\
& & & \\
Frequency of ER visits & $0(0 \%)$ & $1(4.5 \%)$ & .443 \\
Twice a month & $0(0 \%)$ & $2(9.1 \%)$ & \\
Twice a year & $2(67.7 \%)$ & $4(18.2 \%)$ & \\
Once a year & $1(33.3 \%)$ & $15(68.2 \%)$ & \\
Other & & & \\
& & & \\
Extracurricular activities & $3(100 \%)$ & $18(81.8 \%)$ & 1.0 \\
Yes & $0(0 \%)$ & $4(18.2 \%)$ & \\
No & &
\end{tabular}

Note. Fisher's exact test was used to calculate all analyses due to small expected cell counts. Numbers in parentheses indicate the column percentages.

${ }^{\text {a }}$ Stress dose is when the child has to take extra steroids because the body is stressed. In this study, participants stressed dose less than once a year, as needed or had never stressed dose.

${ }^{\mathrm{b}}$ Participants visited the emergency room (ER) less than once a year (i.e., once during childhood) or had never visited the ER $* \mathrm{p}<0.05$. 
Table 4-5. Association between demographic health characteristics and diagnosis

\begin{tabular}{|c|c|c|c|}
\hline \multirow[b]{2}{*}{$\begin{array}{l}\text { Demographic Health } \\
\text { Characteristic }\end{array}$} & \multicolumn{2}{|c|}{ Diagnosis } & \multirow[b]{2}{*}{ p Value } \\
\hline & $\begin{array}{c}\text { Classical } \\
(\mathrm{N}=15)\end{array}$ & $\begin{array}{c}\text { Non-Classical } \\
(\mathbf{N}=\mathbf{1 0})\end{array}$ & \\
\hline \multicolumn{4}{|c|}{$\begin{array}{l}\text { Frequency of Medication } \\
\text { (Hydrocortisone) }\end{array}$} \\
\hline Three times a day & $6(40 \%)$ & $3(30 \%)$ & .773 \\
\hline Twice a day & $2(13.3 \%)$ & $3(30 \%)$ & \\
\hline Once a day & $1(6.7 \%)$ & $1(10 \%)$ & \\
\hline Other & $6(40 \%)$ & $3(30 \%)$ & \\
\hline \multicolumn{4}{|l|}{ Frequency of stress dose } \\
\hline Twice a month & $0(0 \%)$ & $2(20 \%)$ & .062 \\
\hline Twice a year & $6(40 \%)$ & $1(10 \%)$ & \\
\hline Once a year & $2(13.3 \%)$ & $0(0 \%)$ & \\
\hline Other $^{\mathrm{a}}$ & $7(46.7 \%)$ & $7(70 \%)$ & \\
\hline \multicolumn{4}{|l|}{ Frequency of ER visits } \\
\hline Twice a month & $1(6.7 \%)$ & $0(0 \%)$ & .724 \\
\hline Twice a year & $2(13.3 \%)$ & $0(0 \%)$ & \\
\hline Once a year & $3(20 \%)$ & $3(30 \%)$ & \\
\hline Other $^{\mathrm{b}}$ & $9(60 \%)$ & $7(70 \%)$ & \\
\hline \multicolumn{4}{|c|}{ Frequency of communication } \\
\hline Daily & $4(26.7 \%)$ & $0(0 \%)$ & .251 \\
\hline Weekly & $6(40 \%)$ & $3(30 \%)$ & \\
\hline Monthly & $3(20 \%)$ & $4(40 \%)$ & \\
\hline 2-4 times a year & $2(13.3 \%)$ & $3(30 \%)$ & \\
\hline
\end{tabular}

Note. $\mathrm{df}=3$. Fisher's exact test was used to calculate all analyses due to small expected cell counts. Numbers in parentheses indicate column percentages

${ }^{\text {a }}$ Stress dose is when the child has to take extra steroids because the body is stressed. In this study, participants stressed dose less than once a year, as needed or had never stressed dose.

${ }^{\mathrm{b}}$ Participants visited the emergency room (ER) less than once a year (i.e., once during childhood) or had never visited the ER

$* \mathrm{p}<0.05$ 
Table 4-6. Associations between demographic health characteristics and frequency of communication

\begin{tabular}{|c|c|c|c|c|c|}
\hline \multirow{2}{*}{$\begin{array}{l}\text { Demographic Health } \\
\text { Characteristic }\end{array}$} & \multicolumn{4}{|c|}{ Frequency of Communication } & \multirow[t]{2}{*}{ p Value } \\
\hline & $\begin{array}{l}\text { Daily } \\
(N=4)\end{array}$ & $\begin{array}{l}\text { Weekly } \\
(\mathrm{N}=9)\end{array}$ & $\begin{array}{c}\text { Monthly } \\
(\mathrm{N}=7)\end{array}$ & $\begin{array}{c}\text { 2-4 a Year } \\
(\mathrm{N}=5)\end{array}$ & \\
\hline \multicolumn{6}{|l|}{ Frequency of stress dose } \\
\hline Twice a month & $0(0 \%)$ & $2(22.2 \%)$ & $0(0 \%)$ & $0(0 \%)$ & .117 \\
\hline Twice a year & $3(75 \%)$ & $3(33.3 \%)$ & $1(14.3 \%)$ & $0(0 \%)$ & \\
\hline Once a year & $0(0 \%)$ & $1(11.1 \%)$ & $0(0 \%)$ & $1(20 \%)$ & \\
\hline Other $^{\mathrm{a}}$ & $1(25 \%)$ & $3(33.3 \%)$ & $6(85.7 \%)$ & $4(80 \%)$ & \\
\hline \multicolumn{6}{|l|}{ Frequency of ER visits } \\
\hline Twice a month & $0(0 \%)$ & $1(11.1 \%)$ & $0(0 \%)$ & $0(0 \%)$ & .061 \\
\hline Twice a year & $1(25 \%)$ & $1(11.1 \%)$ & $0(0 \%)$ & $0(0 \%)$ & \\
\hline Once a year & $3(75 \%)$ & $1(11.1 \%)$ & $1(14.3 \%)$ & $1(20 \%)$ & \\
\hline Other $^{\mathrm{b}}$ & $0(0 \%)$ & $6(66.7 \%)$ & $6(85.7 \%)$ & $4(80 \%)$ & \\
\hline
\end{tabular}

Note. $d f=9$. Fisher's exact test was used to calculate all analyses due to small expected cell counts. Numbers in parentheses indicate column percentages

${ }^{\text {a }}$ Stress dose is when the child has to take extra steroids because the body is stressed. In this study, participants stressed dose less than once a year, as needed or had never stressed dose.

${ }^{\mathrm{b}}$ Participants visited the emergency room (ER) less than once a year (i.e., once during childhood) or had never visited the ER $* \mathrm{p}<0.05$. 


\section{Research Question 4 Findings}

Responses from the interviews were analyzed using descriptive content analysis. Children's and caregivers' perceptions of HRQOL and factors that may influence the HRQOL of female children with CAH were explored with content analysis. Recurring codes from the child and caregiver interviews were aggregated into categories and themes. The themes that emerged from the child interviews were: 1) living with $\mathrm{CAH} ; 2$ ) normalcy; 3) disclosure of diagnosis; 4) feelings associated with having $\mathrm{CAH}$; and 5) what should we know. The themes that emerged from the caregiver interviews were: 1) living with $\mathrm{CAH}$; 2) feelings of the caregiver; 3) disclosure; 4) what we should know; and 5) improvements.

\section{The Child's Perspective}

Living with CAH. Living with CAH reflects children's experiences of living with $\mathrm{CAH}$ and how they make sense of the situation. Although the responses of each child varied, several recurrent categories emerged. These categories include: 1) knowledge about diagnosis; 2) health; 3) limitations; 4) friends; and 5) adaptation. Knowledge about diagnosis focused on how the child and caregiver first talked about $\mathrm{CAH}$, how the child learned about $\mathrm{CAH}$, and how much the child thinks they know about $\mathrm{CAH}$. Health was defined as children's perceptions of their own health compared to other healthy children their age. Limitations were defined by daily inconveniences and if the child believed CAH kept them from daily activities. Friends were defined by their role in the child's life. School and community focused on the child's sense of belonging to each environment. Adaptation was a latent category defined by strategies the child uses to adapt to living with $\mathrm{CAH}$.

Knowledge about Diagnosis. For all children, knowledge about their diagnosis was dependent on information provided by their caregivers. Children recalled details of early conversations with their parents or caregivers about $\mathrm{CAH}$, and how the caregivers described the condition. It was noted that caregivers in this study associated the information they shared with a child to a symptom the child was experiencing, and the rationale for why the child had to take medication. One child commented, "Well I remember my mom telling me that I am growing too fast. And that by taking this medicine it will help me be as tall as I can be." Another child commented, "... she told me that I have this condition that affects my. . . I think adrenal . . but I'm not sure that's right . . . but it affects my hormones and my growth. And that I would have to take this medication."

Other children noted they could not remember how they first talked about CAH with their caregiver; but they could recall the manner in which the parents described the condition (i.e., at a level where the child could understand). One child stated, "I'm sure she explained it and tried to make [it] to where I could understand it." This participant stated, "She explained it to me in a way that I could understand it. And she told me like I have to take it [medication] at like um in the morning and in the afternoon and at night." 
One adolescent stated that she and her caregiver never talked about having CAH. She said, "I just looked it up one day on my computer. And I'm like oh ... uh this explains a lot. She never actually really told me like what kind of stuff is different." A younger participant made a similar comment. "She doesn't talk to me about that... Well she does a little bit. She says I need to stay healthy."

During discussions about how much children know about CAH, the majority of children believe they did not know much about their diagnosis. Most everyone knew that they were required to take medication and the frequency of the medication. However, few knew the "specifics" or "details" about the disorder so many felt like they had some basic knowledge and understanding about CAH. One child stated, "I mean I know enough to know why I have it and what it does, but I don't really know like the super in depth stuff because there isn't really that much information about it anyway." Another adolescent commented, "I do know some stuff about it ... Like how it's a genetic disorder but not every single detail."

Health. Overall the majority of children believed they were in good health. Perceptions of children's health were described as "good," "normal," "the same," and "almost the same." When discussing health, children compared themselves to their peers. Many believed they were "healthier than other kids." An adolescent stated, "I'm probably healthier. They stay sick. I'm never sick." There were a couple of children who labeled themselves as "sick" or were aware that they get sick more often than other kids. A younger child clearly stated, "Umm... I'm sick." Another participant talked about her health and her experience with frequent hospital visits. She reported, "Well ... I get sick more often. But I do a lot of activities that expose me to it. I don't [think] I'm significantly more unhealthy than them."

Limitations. Children highlighted the limitations and inconveniences that were evident in living with $\mathrm{CAH}$ such as the schedule and timing of medications as well as missing class to take medication. One child stated that the medication regime is "complicated" and gives specific details about her medication schedule: "And just get up in the morning and take my medicine at 2 o'clock in the morning which isn't very fun." This participant voiced her perceptions about the inconveniences when she said the following: "The only thing that bothers me about medicine is like... if I want to stay the night with a friend. I have to take medicine on time so I don't have it all the time ... Basically stuff has to be planned ahead of time." Other comments about the limitations associated with $\mathrm{CAH}$ are described below.

"I mean ... the only thing is I'm sensitive to heat because of it. 'Cus' I get very umm ... like fatigued so I can't like ... it would be kind of scary for me to go like on a long backpacking trip or whatever but that's really it."

"I just think that ... that you do have to be more careful like if you play sports and things like that." 
"I go straight down to the nurse's office and sometimes like if I go down there, I might miss something that the teacher said."

A few children commented that there were no limitations associated with having $\mathrm{CAH}$.

Friends. There were a few comments about the role and relationship of friends in the child's life. Friends helped with medical management and served as a means of support in female children with CAH. One child described how her friends help her with medical management: "And now whenever I go over there she reminds me." Another adolescent stated, "Before I could really understand that I was the same as everybody else, umm my friends would help me with that."

Adaptation. Adaptation was a latent theme identified within the responses of the child interview data. Adaptation was defined by children's attitudes, perceptions, and acceptance in living with CAH. An adolescent participant clearly voiced her attitude by saying, "For me, I try not to think about it very much because it doesn't affect me like in like magnitudes but I kind of have to accept the fact that oh yeah I'm probably gonna have acne for probably a long time and umm like certain things might be a little off." statements:

Children's attitudes and perceptions were also reflected in the following ". . I know that this is something that I will have for the rest of my life."

"It's like I just have this condition that will always be with me... sometimes I think about it, and sometimes I don't."

"I haven't had any major issues so I tend to ignore it usually."

Things that promoted adaptation in children were activities associated with "good days", "bad days" and self-esteem (i.e., what makes the child feel good or bad about themselves). Children's perceptions of good days were associated with the daily activities and daily routines, which usually involved school, home, friends and extracurricular activities. During discussions about bad days, children made comments about "being angry", having a "bad attitude" or "bad mood", not feeling well and being more tired.

When the children were asked questions geared toward self-esteem, responses varied. Overall children felt good about themselves. Sports or extracurricular, accomplishing goals, intelligence, friends, family, and church were all things that made children feel good about themselves and promoted positive adaptation. In contrast, weight, not accomplishing goals or performing well, and name-calling were identified as factors that children perceived to hinder adaptation.

The level of involvement from the child in the management of CAH was an outcome of adaptation. Most children were very involved in the management of $\mathrm{CAH}$, 
which means they were taking medications on their own, with occasional reminders from caregivers and were active listeners at doctor's visits. A couple of children compared their experiences and level of involvement in the initial phases of diagnosis to the present: "When I first tried to take it, it was a little hard but now I'm going on, and starting to take it more and more often. It's starting to get a little easier."

A child expressed a similar comment, "I pretty much take them on my own. When I was younger, my mom would have to remind me . . but now . . . it's just . . . it's just like something I just do."

Normalcy. Normal was a term used to describe children's health identity. During the interviews, children placed emphasis on feeling and being normal with regards to living with CAH. Normal was the child's perception of a normal self, compared to an unaffected child. The following statements were used by children to describe their perception of normal:

"I seem normal ... I just have this condition ... just because they have CAH doesn't mean they can't be normal."

"Uhhh I guess it's like people who don't have CAH. You just have to take medicine and blood draws."

"I'm just pretty normal."

Normalcy also reflected the child's ability to participate in activities in the presence of daily medications and the absence of physical signs of disease. Some children talked about how their days were like "anyone else's", how they continue to play sports, and do things equal to their peers. One child stated, "I can do the exact same things as everybody else. I play sports like everybody else.”

Disclosure of Diagnosis. Disclosure of diagnosis was defined as information sharing. Disclosure of diagnosis focused on the type of information shared, with whom, and how much information was shared. Children talked about the individuals who were aware of their diagnosis, which typically included close family members (i.e., siblings and grandparents), close friends, and school personnel. Family members were more knowledgeable about the child's condition when compared to other people in the child's life. Children found that sharing information was "difficult to bring up the fact that like it's . . . it was seen as like the sex disorder." Many chose to limit the information they told their friends and not "present it fully before saying like what it is exactly." Other children decided not to tell their friends at all. An adolescent strongly commented, "I really don't tell anybody because I don't just like talking about it."

The information that children decided to share was on a "need to know" basis, and was very limited. Children who were heavily involved in sports felt obligated to let at least one friend know in case of an emergency. One child's explanation is as follows, "I tell them about like oh yeah like if I was to like break my arm I might have to get a shot 
of uh ... whatever it is ..." Information that was shared was not "specific", "personal", or "in-depth" according to children. Explanations of the diagnosis ranged from "They just know I have a hormone disorder." to "I just have to tell them I have to medicine 3 or 4 times a day. And that's about it." One participant stated, "Other times I just tell them to Google it because like I kind of really don't want to explain it." Another child explained how she talks to friends who ask about CAH: "Basically they are like well what is CAH? And I said well it's a disease. And they say well what happens and I say well I can't retain salt. That's all I know and that's all I tell them." Only one in the sample commented that talking about CAH was not an "issue" and she was not "ashamed of it or anything."

Feelings Associated with CAH. Feelings associated with having CAH were characterized by how the child internalized their diagnosis. Many children's feelings were imbedded in the social stigma that is associated with having $\mathrm{CAH}$, specifically others' perception of themselves. One child recounts, "It was seen as like the sex disorder or whatever and I try to steer away from that and it's kind of embarrassing...like oh are they gonna find ... are you gonna look up on Wikipedia and see that? Are they gonna judge me? ... I don't know." Another child said that she "just don't want them thinking oh she's sick" or "oh there's something wrong with her." Several children commented that they didn't want people to "look it up" because of how CAH is viewed. Embarrassment was also associated with having CAH. This child stated, "Sometimes it was a little embarrassing in front of a bunch of kids that didn't know me to be the girl that went up there to take medicine in front of everybody."

A younger child expressed her feelings when she stated, "Because they know you take medicine. It would make them feel bad about me."

What Should We Know. Children believed we-health professionals-should know about things they worry about or fear, what is most important in the child's life, and what they think can be done to improve the lives of other children with CAH. Expressions about worries and fears primarily came from older adolescents' responses. Worries and fears were related to performance and the outcomes of having $\mathrm{CAH}$, and one younger child commented about "having her blood drawn." The following comments describes the worries and fears of adolescents:

"Well before a game.. I sometimes think about if I'm going to play well and stuff."

"Umm. . . . sometimes I feel [like] not taking my pill sometimes because I forget, and I fear that umm if I forget and that's not good."

"The anticipation of my height. I'm done growing."

"Like when I grow up maybe not being able to have kids. Or if I miss a dose of my medicine maybe getting really sick and stuff like that." 
Social support from family and friends was an important aspect of children's life. Likewise performing well (i.e., being fit and a good athlete), along with the ability to accomplish goals and tasks was equally important to children. One talked about "church" and the significance of church in the child's life.

The information that children wanted healthcare providers to know varied. Some expressed that the wanted healthcare providers to be familiar with the disorder. Another commented on positive effects of her dosing schedule. Furthermore, many children were very explicit in what they wanted health care providers to know:

"Like don't make extra notice on it. Don't add fuel to the fire about [CAH] ... ooh I have CAH whoo hooo."

"I mean I've never had this issue, but I just think they should treat them like any other kid honestly."

"Like don't make the child feel awkward."

The most liked topic during the child interview was ways to improve the lives of children with CAH. Almost of all of the children provided at least one idea or suggestion to improve other children's life. "De-stigmatizing" the condition, providing opportunities to gain insight into the children's perspective, and making the children feel "normal" were the most frequent suggestions from children. When asked what could be done to improve the lives of children with $\mathrm{CAH}$, the following remarks were made:

"I think definitely de-stigmatizing the disorder because like...like I said before like the sex disorder label can be really offputting to someone who don't know exactly what it is . .. I think it would be helpful to kind of like change up like how we view it. I guess.”

"Maybe more people talking about it so it doesn't have to be so awkward."

"Maybe just letting them know ... uh that just because they have CAH doesn't mean they can't be normal."

Two children made recommendations about medications; for example, "remind[ing] children to take their medicine" and "medicine tasting better" would improve the lives of children with CAH.

\section{The Caregiver's Perspective}

Living with CAH. Caregivers were asked to describe what they think living with $\mathrm{CAH}$ is like and how they make sense of the situation. The majority of caregivers acknowledge that living with CAH impacted some aspect of the child's life. Some described it as "challenging", "a bit stressful", "hard", and "embarrassing." Descriptions of CAH from the caregiver's perspective is revealed in the following statements: 
"Sometimes it may be challenging... having to take medication...or remember to take medication ... And umm having to be more careful with some things. But umm . . overall I don't think it's bad."

"It used to be difficult at times ... going to therapy for her problems . . . dealing with the other issues. Umm . . . but now I don't think it's as difficult... so...it... comes with it's challenges like everything else."

"I think most of the time it's out of her mind, but she's also aware that she is a little bit different and she does protect herself...sometimes that can be hard because she feels different but then on the other hand she's also made some friends who have their own problems."

Similar to the children's responses, the perceptions of caregivers also varied, and several recurrent categories emerged. These categories include: 1) communication; 2) child's health; 3 ) child's management of disease; 4) normalcy; and 5) adaptation. Communication described how the caregiver talked to the child about CAH. Health was defined as the caregiver's perception of the child's overall health. Descriptions of the child's management of disease reflected the caregiver's perceptions of the level of involvement. Normalcy reflects the caregiver's perception of what is normal for a child with CAH. Adaptation was defined as the caregiver's perception of how the child internalizes or externalizes $\mathrm{CAH}$.

Communication. Caregivers began talking to their children about CAH when they felt it was developmentally appropriate or when the child began to ask questions. For some caregivers, conversations about CAH began as early as 6 years old; while others waited until the child was much older. Caregivers told stories of how they were truthful and "matter of fact" with the child. One caregiver states, "I don't think I really talked to her about it until she was approaching her teen years and was starting to show some symptoms, and I talked with her just matter of factly." A father also stated, "I think we were pretty matter of fact. Saying that ok uh well we discovered that there is a uh a condition that's hard to see but uh it has to do with you know your endocrine system."

Accounts of how caregivers explained the diagnosis were also shared. There was no universal language or dialogue used to explain what CAH is to the child. Several caregivers told the child that they have a "hormone disorder" or "medical condition", and that they had to "take medicine."

A caregiver recounted her experience and stated, "I told her that she had a medical condition that affected her hormones, and that she made too much of one hormone. So, in order for her to stay healthy and for her hormones to be at a normal level she has to take medication." Another mom vividly describes her experience in talking with her child as: "I put it in very simple terms. You have a hormonal disorder that umm if there was an emergency and you had a trauma you could ... you know need to go to 
the ...you know you might need some medicine. And you might have to go to the hospital."

Many caregivers expressed that having to explain what is $\mathrm{CAH}$ to a child was "hard" and they didn't "really feel like [they] can give a good explanation of what the situation is." Several commented on the need for universal language to communicate with not only the child, but with other individuals in their life.

Child's Health. Many caregivers believed that overall their child was very healthy. Comparison between children and peers was evidenced by the following descriptions: "she's probably healthier than most kids", "it's about the same", and "maybe even better." One caregiver shared a specific time when CAH affected the child's health. She stated, "The weekend before September 27, 2016, [the child] was with her father at the fair and she passed out ... [We] noticed that she has been complaining more since medications have changed ... [but] other than the CAH, we [are] ok."

Other caregivers felt that their child "gets sick more often" and that when the child gets sick, it usually severely impacts the child.

This particular caregiver stated, "I'd say her health is excellent in the sense of physical strength, uh ability to do different things, uh whatever ... [but] she goes I seem to get sick more than others. [She] sometimes tends to get a bit rundown with some colds that seem to be persistent. That could be related to the condition or could not." Another caregiver made similar comments: "I think on a day to day basis, I'd say she's the same. Umm but when she gets sick . . umm it can be worse. It has a bigger impact on her than it does like with my other kids."

Three caregivers commented on the effects of medical treatment and the child's health, specifically the child's weight. These caregivers notice that the child had gained weight and attributed it to their medication. A caregiver stated, "She's a little bit on the chubbier side which I do think is partially due to the medications and changing medications." The other two caregivers remarked,

"I mean overall [the child] is healthy I think. I mean. The biggest problem with CAH and the health is the weight gain."

"I mean she just got put on birth control and she's gained a lot of weight within the last year ...Like 50 pounds in the last year."

Child's Management of Disease. Caregivers made positive comments about the child's level of involvement in managing CAH. Almost all of the children were involved to some extent with taking daily medication. In the "beginning" children primarily relied on caregivers to manage the daily medication aspect of CAH. However, as children adjusted and were older, they became more self-sufficient. A caregiver recalled, "In the beginning we would have to give her the medicine and make sure she takes it . . . But now she does it on her own. Of course, we still check to make sure you know she's taking 
it every day." Another mom proudly stated, "She's very involved. She has started learning more about it and taking her medicines on her own without us having to remind her."

There were also comments about children becoming self-agents of their own health. Caregivers noticed that their child was taking the initiative to learn more about the disorder, had become an active participant during doctors' visits, and had become more self-aware when they were feeling sick. This was attributed to age and developmental stage of the child. A caregiver reported, "She is a little older she is more involved. I have noticed that she's starting to pay more attention at her doctor's visits." Another caregiver reported similar observations: "She's very involved. She takes her own medication. At her last doctor's appointment, she initiated this. She wants to learn how to give herself her own solucortef shot."

A similar caregiver was very proud of her child's self-awareness and the level of involvement. She proudly claimed, "She's GREAT! She takes her meds. Of course, I'm always [checking] to be sure she's taking her meds and umm ... this is kind of behind her you know ... and she... but she takes them regularly. Rarely does she forget... Because she's aware that she has her disorder. And so she tries to get enough sleep. She tries to eat right. She tries to be hydrated. She avoids hot places. Like she knows her triggers."

Normalcy. Normal was a recurring term used by caregivers to describe the child's ability to live a life equal to their peers without being stigmatized. Caregivers attributed normal to the child's identity compared to other children, to describe "a good day", and to describe how they treated their child. A caregiver stated, "I don't treat her any different... So I kind of just raised her like a normal child." Another caregiver expressed the paradoxical statement: "You are sick but you are normal." Normal was also attributed to the day-to-day activities of children. "A good day is a normal day," stated one caregiver. According to another caregiver normal is "like any other day. Her routine is... well she gets up goes to school, hangs out with her friends comes home... Just a normal day."

Adaptation. Caregivers viewed adaptation as a process that involved learning how to adjust and accept the situation. From the caregiver's perspective, adaptation was reflected in the child's behavior, attitudes, and self-esteem. Caregivers commented that CAH just became a part of who they were and that they had "just learned to live with it." A mother recounts her child's process of adaptation by saying, "I think she was able to get through that diverse... umm not diversity but adversity and she started taking the dexamethasone and some other drugs and her skin is cleared and she's still that same sweet girl even though she had that hard time." A similar response came from a caregiver: "But they're pretty adaptable. So, they kind of got into the 'Oh well, it's just a part of who I am and what I do."”

Adaptive methods of dealing with CAH were exhibited in the child's attitude, behavior, and ability to connect and engage in social activities with others. Some 
parent's made comments about the child "staying busy" and "engaged" which were positive and occurred during "good days." For one child, a good day was when she was "so engaged, energetic, involved with her friends, doing what she likes to do." Likewise, another parent felt that good days were when "She goes to church. She talks to her friends. She comes home. She does music. She listens to music. She hangs out with her sister. She may go hang out with her friends."

In contrast, negative attitudes and behaviors occurred during "bad days." During bad days, children were "moody", not really engaged, "worn out," and "irritable." A mom described it as: "well her typical bad is when [she's] moody or gets upset about something... And it's not easy to get her out of that mode." Two caregivers were transparent and remarked, "She can get a little moody, and I'll always kind of blame that on the steroids or the CAH." She has a "bad attitude. Umm yeah ... She's hard headed. You just can't tell her anything."

Self-esteem was a topic that caregivers also discussed-specifically what makes the child feel good or bad about herself. Overall, caregivers thought that children feel good about themselves. Sports, being physically fit, family, friends, and accomplishments were all things caregivers believed influenced self-esteem and fostered adaptation. A different response came from a caregiver of an older adolescent: "I think she feels good about how far she's come in terms of accepting who she is and how she looks." A few caregivers also acknowledge that their child enjoyed be recognized for their "accomplishments."

Caregivers identified very few things that made the child feel bad about herself. Weight gain, wanting to "fit in", and "wanting the attention and approval of their siblings" were associated with negative self-esteem. A caregiver stated, "If you say she looks different she doesn't like that. She says why am I bigger than this one....Her teacher says she just cries, and those two little girls keep picking with her." Likewise, another child caregiver mentioned, "It's been a while since she's mentioned anything. But I think her weight sometimes gets brought up."

Feelings of Caregiver. Feelings of the caregiver were characterized by emotions associated with caring for a child with CAH. Caregiver's emotions and feelings varied at different time points in the child's life. Caregivers recounted "being born with something so rare was overwhelming at first." Other parents similarly reported feeling overwhelmed and anxious. One caregiver admitted, "It was very upsetting. I took it harsh. It's upsetting even though I'm personally experiencing — not the same situation but you know obviously it's not the same condition." Another caregiver expressed that it was "scary", and she felt "bombarded" with a wealth of information about CAH. After some time, caregivers' emotions shifted from anxious and being overwhelmed to concerned, fearful, "cautious," and "protective."

A few caregivers were angry and concerned about the surgical outcomes. These caregivers remarked: 
"The only thing that I'm really concerned about is before she had surgery (it probably has nothing to do with it) but before she had surgery she uh . . . didn't wet the bed. But since she has had surgery she wets the bed."

"A few doctors, not many, have made comments about doing the surgery. I'm just not a big proponent. Well first off she will be a woman one day and I don't want to take any chance of taking that away from her."

"What pissed me off about the surgery is that it wasn't done right. Even though I know the mom violated the child's right by not taking her back to that doctor. This is wrong. This is wrong."

Furthermore, the same caregiver who "took it harsh" continued, "And then my other fear was, Is this going to screw her up?. You know what I mean. Is it going to jeopardize her health? Is she going to be able to get her period? Is she going to be able to get married? You know have children." There were several things that caregivers feared including how others would view their child and what would happen in an emergency situation. One caregiver was transparent by saying, "I didn't want her viewed as different." Another parent reported that she did not want her child to "look it up" because of all the "bad things women were saying."

Others expressed fears about emergency situations and "having the support" to care for their child. This fear led most caregivers to be "protective" and cautious." A caregiver goes into details about her fears:

"The fear of [needing] the injection. I don't feel comfortable because I don't think they could fully take care of her the proper way. And a parent like me is too far away to be there do anything right away. So, you just have that fear in the back of your mind. Will they... do it (give the injection) on time? Do they care enough?" Another caregiver commented, "Is there always that fear of what if she gets sick and I'm not there? Of course."

Additional emotions and feelings that caregivers reported were guilt, blame, and isolation. A couple of caregivers blamed someone or something else for the child's diagnosis, which also made them feel "sorry" for the child. These caregivers expressed that they felt "bad" for not doing something sooner. Several caregivers also mentioned that they felt "alone" or really had no one to talk to. However, only one caregiver specifically expressed how they felt in the following statement: "And you feel alone. You really do because you don't know anyone else with the condition. And you don't have anyone to turn [to] and talk about what you had to deal with today."

Disclosure of Diagnosis. Similar to the child's theme, disclosure of diagnosis focused on information sharing. Disclosure of diagnosis involved the type of information shared, with whom, and the manner in which it was shared. Caregivers had no problem disclosing information to their child. As noted in a previous section, caregivers were truthful, honest, and willing to answer and share as much information as the child wanted 
to know. However, caregivers were more hesitant to disclose the child's diagnosis to anyone outside the immediate family. According to the caregivers, only a few people outside the immediate family-usually grandparents or aunts-knew that their child had CAH. A few of the caregivers' friends and the parents of the child's friends also knew about the diagnosis. Still, several people outside of the immediate family who were aware of the child's condition did not know the child had CAH, but they knew the child needed to take medication or had a "hormone disorder."

The majority of caregivers used the term "adrenal insufficiency" instead of CAH when they were explaining the child's diagnosis to individuals outside of the immediate family. A caregiver openly commented, "When they ask, she said, 'We don't advertise CAH.' We just say, 'It's adrenal insufficiency." Also, many caregivers chose to limit the information they disclosed about "adrenal insufficiency" because it was "private." Caregivers placed emphasis on the fact that CAH is not a "secret" but it's private. It's not something that's really shared with or talked about with others. One caregiver was different than any other during discussion about disclosure. Her response was the opposite of all of the caregivers: "And I just feel like it's almost the secrecy that a lot of families umm aren't open and honest and they aren't given the comfort level to be...I just wish it wasn't so secretive."

What We Should Know. Caregivers provided information about what the child worries about or fears, what is most important to the child and what they-the caregiverswould want health care professionals to know. Caregivers found it challenging to identify what the child worries or fears with regards to CAH. A few mentioned, ". . . mainly working hard to reach her goals" and the ability to continue playing sports.

Caregivers reported that social support from friends and family was most important to the child. They believed that being able to connect, and interact within that social network without fear of being stigmatized was essential to the child's life. A male caregiver remarked, "Well to have friends in school, to go do sports, to do something with music . . . to be connected. I think that's important. I think she'd say that's something she needs to do."

The most talked about topic and important thing caregivers thought healthcare professionals needed to know centered on education. All caregivers described at least one encounter with a health care provider who was not "familiar" with CAH or knew how to adequately treat $\mathrm{CAH}$. Caregivers admitted that there were situations in which they "educated the provider" about the child's diagnosis and the solu-cortef. They expressed frustrations about the lack of general knowledge between providers about CAH. A caregiver stressed, "Ummm I would want them to understand what adrenal insufficiency is. Umm not even necessarily CAH. That's just a type of adrenal insufficiency. But I would want them to kind of know the basics of how to handle umm a child with an adrenal insufficiency." Similar comments from caregivers were:

"They know nothing about CAH. Umm I just go in with adrenal insufficiency. I provide them with literature. Umm... uh they know nothing. I mean ER visits are a disaster." 
"I just want them to be educated. They don't have to know all of the specifics, but if I bring my child into the ED or office I need you to know what it is and what to do."

One caregiver discussed her experience, and she talked about the significance of only ordering what's necessary. She experienced the ramifications of an expensive insurance. Another caregiver commented, "All kids are not the same," so treatment should be tailored to fit the needs of the child.

Improvements. The rarity of $\mathrm{CAH}$ led caregivers to believe that much could be done to improve the lives of children with $\mathrm{CAH}$. Education, social acceptance, research, camps, financial support, and support groups were thought to improve children's lives. As previously mentioned, caregivers were frustrated about the lack of education between health care providers so more education for providers was listed at the top for improvement. More specifically, caregivers wanted health care providers to understand that CAH can be life threatening so they should "at least be familiar" with CAH and have a "sense of urgency" about the situation.

One caregiver expressed, "It's the same sort of situation almost. Umm so I don't know if it's a literature thing or what. And there's so many doctors that don't know what it is. That's crazy, and scary because I think one day she's going to be on her own and end up [in] the ER or somewhere where they have no idea what that is. So I guess the biggest thing is education." Several other caregivers believed "there's just not enough of it out there" and "important places would be like emergency room, emergency response people."

The topic of education was not limited to health care professionals. Caregivers also believed that there should be more education for themselves and "a way to explain it $[\mathrm{CAH}]$ to others."

A mom gave a detailed description about the importance of education:

"I can read up on it on the computer all day you know. But if I don't have... I dunno. I'm not one to put my face in books, and I don't care to read so it's hard for me [to] really get into it. So, if I had more people to... reach out to I feel like I would be more educated... But I would love for there to be an easier way to explain it and educate other people [and] something that educates siblings." She also suggested that doctors or [another healthcare professional] should educate teachers and school personnel.

One caregiver voiced concerns about solu-cortef, "the injection," not being readily available on Emergency Medical Service (EMS) Vehicles.

Caregivers also made comments about the treatment of CAH. A few hoped for a cure; while others wanted ways to better manage the medications, improve surgery, and a better way to "monitor the child's status." A mother expressed excitement because she "[heard] of longer acting medication so that it doesn't have to be three times a day or 
whatever that it could be one time a day" and felt that that would make her child's life better.

During conversations about improvements, one caregiver was very saddened and tearful that her child had to live with $\mathrm{CAH}$ and experience the side effects of taking steroids. She stated, "Steroids ... that's a hard medication on a child. And with her being her age. It's hard on a child ... And it's hard. And my [the child's name] it breaks my heart. [pause] [tearing up] It really breaks my heart." She too hoped for a better way to manage $\mathrm{CAH}$.

Social support in the form of support groups for children, caregivers, and families was believed to also improve the lives of children. Several caregivers mentioned that, "more needs to be done with the kids", and "it would be nice to have another kid's perspective on what they are going through, and a little more understanding for the parents." Caregivers felt that caring for a child with CAH was a "huge responsibility" and "hard when you feel like you are the only one ..." Caregivers were aware of and mentioned resources from the CARES foundation; however many could not afford to travel due to financial burdens and "other situations."

Several mothers remarked,

"I would love to hear other stories and talk to other parents. You know ... see how they deal [with CAH]."

"You know that's why I do hope we do get some support groups there so we will be able to communicate more with it other than just going to the doctor's office."

"It would be nice to have a type of support group. Especially for parents when it's their first child, especially with that. Or like some type of once a month thing where parents can get together and the kids can get together and talk about things that they might be experiencing."

Lastly, social acceptance and more research were believed to impact the lives of children with $\mathrm{CAH}$. A caregiver, who happens to be a support group leader, commented, "... just that if we can open up and embrace the information and the umm study groups and things that are out there it helps the ... the whole ummm country as we all learn how to deal with CAH." Many caregivers desired open communications about what CAH really is and social acceptance from society so that children would not feel different. So, many believed that "Just the fact that you have taken an interest . . . an interest [in] CAH is a start."

\section{Specific Aim Four}

The last aim was to compare the quantitative and qualitative child and caregiver results on HRQOL of female children with $\mathrm{CAH}$. 
Research Question 5: To what extent do the themes on HRQOL emerging from interviews with children support the self-reported HRQOL as measured by the KINDL-R?

Research Question 6: To what extent do the themes on HRQOL emerging from interviews with caregivers support the caregiver proxy-reported HRQOL as measured by the KINDL-R?

Research Question 7: To what extent do the themes on HRQOL emerging from interviews with children and their caregivers support the self-report and caregiver proxy-reported HRQOL as measured by the KINDL-R?

\section{Research Question 5 Findings}

Independent analysis and interpretation was conducted on both the QUAN and QUAL data. The findings were integrated to support a mixed methods design which is displayed in Figure 4-1. Comparisons and contrasts between the findings were carried out to identify common themes, categories, and subject matter. Results were displayed in a side-by-side joint display table to complete the merging of data (Creswell, 2014). QUAN variables were compared and merged with QUAL final themes and categories. Merging of data showed that not all of the QUAN variables are reflected in the QUAL themes and vice versa. However, many of the QUAL categories and comments elucidated information about the spectrum of children's and caregivers' perceptions about the HRQOL of children with CAH.

Table 4-7 displays a comparison of the child QUAN interview data and the QUAL KINDL-R scores.

Overall children reported they had a good HRQOL. Younger children and older adolescents' perception of HRQOL varied and were revealed by their experiences in living with $\mathrm{CAH}$. Children understood health to be the ability to physically function every day without "getting sick." When asked about their health, children believed that they were "healthier than most" and "didn't get sick as much" which supports the QUAN results of the Physical Well-Being subscale scores. QUAN analysis of the Physical WellBeing scores was also reflected in the child's ability to adapt to having CAH by staying physically active. Many children described the importance of being active, playing sports, and staying fit.

Children scored highest on the Emotional Well-Being subscale. However, during the QUAL analysis children described different emotions and feelings that were associated with having CAH such as embarrassment and being fearful of other's perception of CAH. Children's Emotional Well-Being was also dependent on whether the child was having a good or bad day. Divergent results also appeared from QUAN and QUAL analysis of Self-Esteem from the child's perspective. Children reported lower scores in the Self-Esteem subscale of the KINDL-R. During the interviews, children 


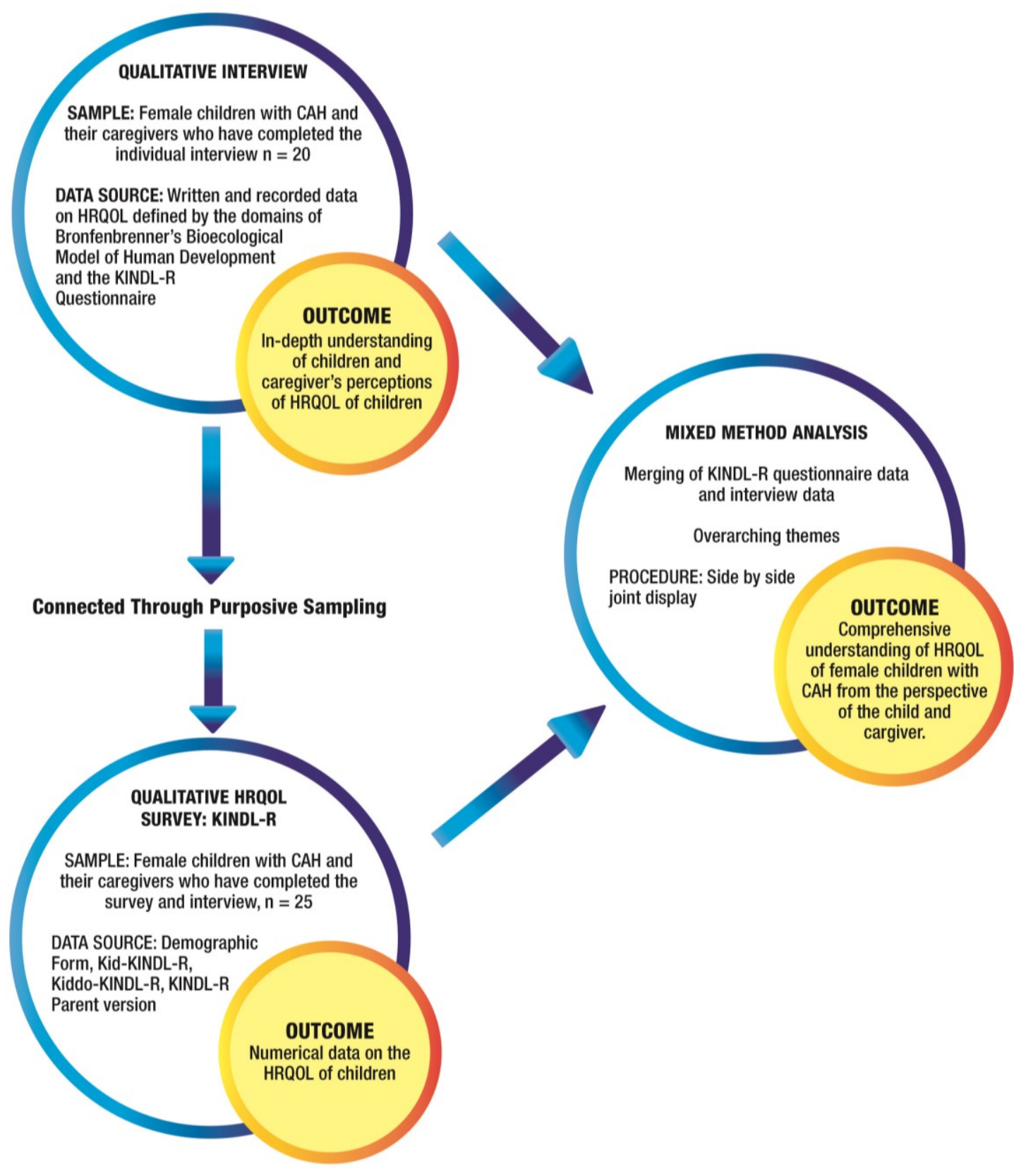

Figure 4-1. Mixed methods convergent design of HRQOL of female children with CAH 
Table 4-7. Comparison of the child QUAN interview data and the QUAL KINDL-R scores

\begin{tabular}{|c|c|c|}
\hline KINDL-R Subscale & $\begin{array}{l}\text { Qualitative Theme and } \\
\text { Category }\end{array}$ & $\begin{array}{l}\text { Similarities and } \\
\text { Differences }\end{array}$ \\
\hline Physical Well-Being & $\begin{array}{l}\text { Living with CAH } \\
\text { - Health } \\
\text { - Adaptation }\end{array}$ & $\begin{array}{l}\text { Physical Well-being was } \\
\text { not impaired as evidence } \\
\text { by the child's ability to } \\
\text { adapt to CAH by their } \\
\text { level of involvement in } \\
\text { physical activities. }\end{array}$ \\
\hline Emotional Well-Being & $\begin{array}{l}\text { Living with } \mathrm{CAH} \\
\qquad \text { Adaptation } \\
\text { Feelings associated with having } \\
\text { CAH }\end{array}$ & $\begin{array}{l}\text { Emotional Well-being } \\
\text { received the highest } \\
\text { scores. However, during } \\
\text { the interviews, } \\
\text { children's emotions } \\
\text { varied on good and bad } \\
\text { days. Children felt that } \\
\text { CAH could be } \\
\text { "embarrassing." }\end{array}$ \\
\hline Self-Esteem & $\begin{array}{l}\text { Living with CAH } \\
\text { Normalcy }\end{array}$ & $\begin{array}{l}\text { Self-esteem was one of } \\
\text { the lowest scoring } \\
\text { subscales. Children } \\
\text { wanted to be "normal." } \\
\text { However, when asked } \\
\text { specific questions about } \\
\text { self-esteem, children } \\
\text { reported that overall, } \\
\text { they felt good about } \\
\text { themselves. }\end{array}$ \\
\hline Family & $\begin{array}{l}\text { Living with } \mathrm{CAH} \\
\bullet \quad \text { Communication } \\
\text { Disclosure of diagnosis }\end{array}$ & $\begin{array}{l}\text { Family was not } \\
\text { impaired. Most served as } \\
\text { support and vehicles of } \\
\text { information for children } \\
\text { with CAH. }\end{array}$ \\
\hline
\end{tabular}


Table 4-7. (Continued)

\begin{tabular}{|c|c|c|}
\hline KINDL-R Subscale & $\begin{array}{l}\text { Qualitative Theme and } \\
\text { Category }\end{array}$ & $\begin{array}{l}\text { Similarities and } \\
\text { Differences }\end{array}$ \\
\hline Friends & $\begin{array}{l}\text { Living with CAH } \\
\bullet \quad \text { Friends } \\
\text { Disclosure of diagnosis }\end{array}$ & $\begin{array}{l}\text { The Friends received } \\
\text { the highest scores. } \\
\text { Friends had several } \\
\text { roles and were some of } \\
\text { the most important } \\
\text { people in children's } \\
\text { lives. }\end{array}$ \\
\hline $\begin{array}{l}\text { School/Everyday } \\
\text { Functioning }\end{array}$ & $\begin{array}{l}\text { Living with } \mathrm{CAH} \\
\bullet \quad \text { Limitations }\end{array}$ & $\begin{array}{l}\text { School subscale } \\
\text { received the lowest } \\
\text { scores. Very few } \\
\text { children mentioned the } \\
\text { effects of school and } \\
\text { everyday functioning } \\
\text { Children did talk about } \\
\text { the limitations and } \\
\text { inconveniences of } \\
\text { CAH. }\end{array}$ \\
\hline Total HRQOL & $\begin{array}{l}\text { Living with CAH: } \\
\text { - Health }\end{array}$ & $\begin{array}{l}\text { Overall good HRQOL } \\
\text { on KINDL-R. Children } \\
\text { reported they were } \\
\text { "healthier than most } \\
\text { kids" and their health } \\
\text { was "good." }\end{array}$ \\
\hline
\end{tabular}

Note: The content in bullet point in the second column represents the categories; nonbulleted content represents themes. 
expressed a strong desire to be viewed as "normal" and treated as such. When asked specific questions about how they felt about themselves, children stated, "They feel good [about themselves]."

The Friends subscale also received higher scores from children with CAH. QUAL results showed that children had at least one friend they felt comfortable talking to and sharing information with. Friends served as support systems for children with CAH. More often than not, children listed friends as some of the most important people in their life, which was apparent in QUAN results. The Family subscale scores indicate that there family did not hinder or impair this aspect of HRQOL. Family, more specifically, caregivers served as vehicles of information and support for children with CAH.

The School subscale received the lowest scores from children with $\mathrm{CAH}$. There were very few comments about the effects of school and everyday functioning. However, children did talk about the limitations and inconveniences of $\mathrm{CAH}$ with respect to school. A few children discussed how taking medication causes them to miss class and the effects of taking medication in front of students or friends. For example, children made remarks about how they feel embarrassed or awkward when they have to walk out in the middle of class to take medications.

\section{Research Question 6 Findings}

Table 4-8 displays a comparison of the caregiver QUAN interview data and the QUAL KINDL-R scores.

Caregivers reported that the overall HRQOL of children with CAH was not impaired. QUAL analysis revealed that caregivers often compared their child's "health" and Physical Well-Being to children without CAH. The child's management of the disease was also important to the overall HRQOL from the caregiver's perspective. Parents emphasized the importance of managing the disease in specific situations. Caregivers also compared the child's health at the beginning of diagnosis versus the child's current health. Thus, time was a fundamental component of HRQOL and Physical Well-Being. Additionally, analysis revealed that Physical Well-Being was related to the QUAL theme, "normalcy." Caregivers expressed that children were "normal" and they [the child] are just like any other child.

Caregivers reflected on their own personal feelings of caring for a child with CAH. QUAN analysis revealed that the Emotional Well-Being received the highest scores from caregivers. However, the QUAN analysis may not accurately reflect the child's Emotional Well-Being. Moreover, similar to the perceptions of children, friends and family were integral in the lives of the child with CAH and their caregiver. This was reflected in the QUAN scores of the Friends and Family subscales. Friends and family helped provide care as well as support for children with $\mathrm{CAH}$ and their caregivers. Other QUAL categories associated with friends and family were disclosure of diagnosis and 
Table 4-8. Comparison of the caregiver QUAN interview data and the QUAL KINDL-R scores

\begin{tabular}{|c|c|c|}
\hline KINDL-R Subscale & $\begin{array}{l}\text { Qualitative Theme and } \\
\text { Category }\end{array}$ & $\begin{array}{l}\text { Similarities and } \\
\text { Differences }\end{array}$ \\
\hline Physical Well-being & $\begin{array}{l}\text { Living with CAH } \\
\text { - Child's health } \\
\text { - Normalcy }\end{array}$ & $\begin{array}{l}\text { Caregivers expressed that } \\
\text { children were "normal" } \\
\text { and [the child with CAH] } \\
\text { are just like any other } \\
\text { child. }\end{array}$ \\
\hline Emotional Well-Being & Feelings of caregiver & $\begin{array}{l}\text { QUAN analysis revealed } \\
\text { that the Emotional Well- } \\
\text { Being received the highest } \\
\text { scores from caregivers. } \\
\text { However, the QUAN } \\
\text { analysis does not } \\
\text { accurately reflect the } \\
\text { child's emotional ell- } \\
\text { being. }\end{array}$ \\
\hline Self-Esteem & $\begin{array}{l}\text { Living with CAH: } \\
\text { adaptation }\end{array}$ & $\begin{array}{l}\text { Self-esteem was not was } \\
\text { impaired. Caregivers } \\
\text { believed that children felt } \\
\text { good about themselves. } \\
\text { Sports, being physically } \\
\text { fit, family, friends, and } \\
\text { accomplishments were all } \\
\text { things caregivers believed } \\
\text { influenced self-esteem and } \\
\text { fostered adaptation. }\end{array}$ \\
\hline Family and Friends & $\begin{array}{l}\text { Feelings of caregiver } \\
\text { Disclosure of diagnosis }\end{array}$ & $\begin{array}{l}\text { Caregivers agreed that } \\
\text { friends and family were } \\
\text { integral in the HRQOL of } \\
\text { children with CAH. } \\
\text { Additionally, they were } \\
\text { often the first and only to } \\
\text { know about the child's } \\
\text { diagnosis. }\end{array}$ \\
\hline
\end{tabular}


Table 4-8. (Continued)

\begin{tabular}{lll}
\hline KINDL-R Subscale & $\begin{array}{l}\text { Qualitative Theme and } \\
\text { Category }\end{array}$ & $\begin{array}{l}\text { Similarities and } \\
\text { Differences }\end{array}$ \\
\hline $\begin{array}{l}\text { School/Everyday } \\
\text { Functioning }\end{array}$ & $\begin{array}{l}\text { Caregivers scored lowest } \\
\text { on the School subscale. } \\
\text { However, they did not } \\
\text { provide in depth QUAL } \\
\text { responses about the } \\
\text { impact of school on the } \\
\text { child's HRQOL. }\end{array}$ \\
& $\begin{array}{l}\text { Caregivers felt that } \\
\text { children's HRQOL was } \\
\text { not impaired. QUAL } \\
\text { analysis were similar to } \\
\text { children's perceptions in } \\
\text { that caregivers believed } \\
\text { the child was healthy } \\
\text { compared to children } \\
\text { without CAH. }\end{array}$ \\
& $\begin{array}{l}\text { Child's health } \\
\text { Child's management } \\
\text { of disease }\end{array}$ & \\
&
\end{tabular}

Note: The content in bullet point in the second column represents the categories; nonbulleted content represents themes. 
things that are most important to the child. Caregivers agreed that friends and family were often the first and only to know about the child's diagnosis.

Caregivers scored lowest on the School subscale. However, they did not provide in depth QUAL responses about the impact of school on the child's HRQOL.

\section{Research Question 7 Findings}

Both children and their caregivers believed the overall HRQOL of female children with $\mathrm{CAH}$ is not impaired. However, there are certain factors that contribute to different aspects of a child's HRQOL. Children and caregivers perceived physical wellbeing as the ability to function in the presence or absence of disease. For children, QUAN analysis supports the QUAL responses that focused on Physical Well-Being. Although, time was an important factor in caregivers' perceptions of children's Physical WellBeing; the QUAN analysis did support the QUAL analysis and children's perceptions about their Physical Well-Being. With regards to Emotional Well-Being, Self-Esteem, Friends and Family, the QUAN results for child and caregivers supported the QUAL findings. Additionally, there was a statistically significant difference in the child and caregivers' perception of school in the QUAN results. Though, caregivers did not provide many comments about the impact of school, children gave specific examples of the impact of CAH on their school activities. 


\section{CHAPTER 5. DISCUSSION}

Over the last decade QOL as a measure of therapeutic outcomes has become more popular, especially in individuals with CAH and DSD. However, few studies have explored children and their caregivers' lived experiences regarding CAH and HRQOL. This study examined the HRQOL and factors that may contribute to the HRQOL of female children with CAH from the child's and caregiver's perspective. This chapter provides a discussion of the results and the implications of the study.

\section{Demographics}

The participants in this study were 25 female children and 25 caregivers living in several states across the United States including, Tennessee, Arkansas, Mississippi, Kentucky, California, New York, Oregon, Illinois, Virginia, Arizona, Colorado, Massachusetts, and Vermont. The 25 female children were between the ages of 7 and 18 years of age, and the 25 caregivers were 18 years and older. The age distribution is similar to that in existing HRQOL studies with adults and children with $\mathrm{CAH}$ (Jurgenseen et al., 2014; Yau et al., 2015). Children and their caregivers were largely Caucasian (54\%) with lower representation of Black or African American (24\%) participants, American Indian/Asian, and other (22\%) participants. The racial distribution in this study is not consistent with findings from other HRQOL studies in children and adults with CAH or DSD. These studies include samples of individuals who are largely Caucasian, Asian and other races that are attributed to the country in the study was conducted (Amaral et al., 2015; Bennecke et. al., 2017; Engberg et al., 2016; Gilban et al., 2014; Yau et al., 2015). This discrepancy may be attributed to the recruitment technique, access to larger databases in European countries, and prevalence of the disorder in certain ethnic and racial groups.

Most of the caregivers in this study were married (68\%), had at least two years of college education or more, and were employed full-time. The marital, educational, and employment distribution of this study is not consistent with the few HRQOL studies that exist (Strandqvist et al., 2014; Yau et al., 2015). This may be attributed to the fact that much of the research with individuals with CAH has been conducted in other countries. This may also be attributed to the nature of the research study and outcomes (i.e., surgical outcomes and effects of treatment and management). Additionally, it may be attributed to the fact that studies that examine the HRQOL of female children with CAH are scarce, and the few that do exist do not include children and their caregivers.

\section{Specific Aim One}

The first specific aim was to examine the HRQOL of female children with CAH. The following research question pertains to the first specific aim: 
Research Question 1: What is the HRQOL of female children with CAH as reported by:
a. children with $\mathrm{CAH}$ ?
b. caregivers of children with $\mathrm{CAH}$ ?

As a group, children reported lower total HRQOL scores compared to their caregivers, which indicates that the HRQOL of female children from the child's perspective is slightly impaired (Bullinger et al., 2008). According to Jurgensen and colleagues (2014), impaired HRQOL in children with CAH could be explained by significantly lower scores in the school domain, diminished self-esteem, and to a lesser extent physical well-being (Jurgensen et al., 2014). In this study, children also reported lower scores in the Self-Esteem and School subscales when compared to their caregivers. In the extant literature on HRQOL of female children with CAH, Yau et al. (2015) also determined that the HRQOL of female children with CAH is adversely affected. Similar to the results of the present study, Yau et al. (2015) observed that children reported lower scores in the physical, emotional, social and school functioning domains of the PedsQL (Yau et al., 2015). Gilban et al. (2014) also explored the HRQOL of children with CAH and noted that the self-assessment scores were significantly lower in the physical, psychosocial and total scores (Gilban et al., 2014). Based on the findings of this study, clinicians may now acknowledge the need for assessments beyond physical symptomology. More specifically, clinicians may recognize the need for interdisciplinary assessments that focus on social and psychological aspects of care to further improve the overall HRQOL of children with CAH.

The results of the present study as well as the aforementioned studies may be a reflection of developmental stage of the child in addition to how well the child copes and adapts to having CAH. In a review by Taylor, Gibson and Franck (2008), one of the most prominent factors that affected children's perceptions of HRQOL was development. Jurgensen et al. (2014) also suggests that development provides insight into a child's well-being by considering the timing of and ability to adjust to current "life circumstances" (Jurgensen et al., 2014). Children in this study were required to respond based on their experiences in the last week. So, perhaps the HRQOL scores were influenced by time, perception, and memory. Also, developmental experiences and tasks vary between, for example, a 7-year-old and 15-year-old, such as the role in peer and social interactions, and level of independence in managing CAH. Hence, HRQOL is experienced differently at different stages in life. Taylor et al. (2008) agrees that the developmental stage of the child influences certain perceptions such as the biological, psychological, and social aspects of HRQOL (Taylor et al., 2008).

Furthermore, caregivers reported no impairment in children's overall HRQOL. For caregivers and children in this study impairment was described as good, average, or poor. The results of the present study suggest that caregivers overestimated children's physical and emotional well-being as well as friends, but underestimated their self-esteem and school domains. Perhaps this reveals true differences in child and caregiver perspectives and the caregiver's limited knowledge about the internal problems children 
with CAH experience. It has been documented that caregiver and proxy respondents are more able to assess external problems (i.e., functioning, behavior) and are usually less accurate in judging internal problems (Eiser \& Morse, 2001a; Jokovic et al., 2004). Hence, it may be reasonable to conclude that caregivers are more accurate in rating physical aspects of HRQOL than less observable domains such as Self-esteem and Emotional Well-being.

\section{Specific Aim Two}

Research Question 2: Are there any differences between child self-report and caregiver proxy-report KINDL-R scores?

Research Question 3: Are there any associations between demographic health factors and HRQOL graphic rating scores as reported by caregivers of children with CAH?

There were no significant differences between the child-self report and caregiver proxy-report of total HRQOL scores. However, children's and caregivers' perceptions of school significantly differed. A few children expressed the impact of living with and managing $\mathrm{CAH}$ in school and in activities outside the home. Children's interview data revealed the challenges associated with managing $\mathrm{CAH}$ at school such as missing class to take medication, perceptions of school educators and staff, and the stigma associated with peers knowing. Perhaps, it is likely that caregivers lack insight into the impact of contextual variables on children's internal feelings (Pickard \& Knight, 2005). Upton, Lawfor, and Eiser (2008) suggest that the differences may provide understanding by highlighting the lack of caregiver knowledge about children's experiences or it may indicate that there is a difference in the perception of self and others. A review that examined children's experiences of living with chronic illnesses highlighted how children's lives were affected by their school experience. Many felt that having a chronic illness affected their academic success. Specifically, school absences interfered with learning and social relationships, which led to social isolation, and children withheld their diagnosis because they wanted to be judged as equal to their classmates (Taylor, Gibson, \& Franck, 2008b).

The difference in School subscale scores between children and caregivers may also be attributed to children's experiences with school personnel. Children spend most of their time in school; however, many educators and school nurses lack adequate knowledge and training about the needs of children with conditions like CAH. Much of what the child experiences in the classroom may occur from school personnel attitudes, biases, or other experiences. Studies show that school personnel typically express concerns about the risk and liability of caring for a child with a chronic illness. School personnel are also concerned about how to respond in a medical emergency, the impact of disease on academic performance, behavioral issues, the demand for more time or attention, as well as the impact of disease on others in the classroom (Hinton \& Kirk, 2015; Olson, Beildler, \& Goodman, 2004). Thus, understanding school personnel's 
attitudes and concerns may provide clinicians with insight into children's experiences, allow the development of programs to support school personnel, and ensure that children with $\mathrm{CAH}$ are receiving adequate support at school (Hinton \& Kirk, 2015).

It is also important to consider the idea that discrepant perspectives may reflect methodological issues. In the present study, the differences of the school aspect of HRQOL violates the long standing assumption that child and caregiver responses should "match" (Eiser \& Morse, 2001a). Previously, child and caregiver discordance were often labeled as methodological errors, which led to disputes about who is right or wrong (Eiser \& Varni, 2013). However, current interpretations suggest that less attention be given to who is right or wrong, and more attention should be given to the clinical meaning of the discordance (Eiser \& Varni, 2013; Taylor et al., 2008b; Upton, Lawford, $\&$ Eiser, 2008). Both child and caregiver perspectives are important to consider and can contribute to the understanding of HRQOL in female children with CAH.

Generally, caregivers believe that an illness or disorder has more effects on children. In the current study, there was also a relationship with caregiver's belief about the child's other health issues and its effects on HRQOL. Some caregivers in this study reported that the child's diagnosis (classical versus non-classical) and other health issues affected the overall HRQOL of children with CAH. Perhaps children with classical CAH had worse HRQOL than children with non-classical CAH. Perhaps parents believed the clinical course and the management of classical CAH is more challenging that having non-classical. Nordenskjold et al. (2008) found that adults with classical CAH usually have a worse HRQOL than adults with non-classical. In the current study, there was not significant relationship between the frequency of communication about $\mathrm{CAH}$ and number of times the child had to stress dose and visit the ER. To date, the relationship between the frequency of communication, the frequency of stress doses (how many times the child has to take extra steroids because the body is stressed), and frequency of visits to the ER has only been supported in one study. Engberg and colleagues (2016) loosely discuss this association when they describe women with $\mathrm{CAH}$ experiences with the care they received. Many women had discussed how the frequency of ER visits affected their life in many ways from not really understanding why visits were needed to feeling like the ER was a "safe haven". Women in this particular study also expressed concerns about the inability of physicians and parents to communicate information, which made them feel like they were not self-agents managing their own health (Engberg, Risen, Moller, Hagenfeldt, \& Nordenskjold, 2016).

\section{Specific Aim Three}

The third aim was to explore the HRQOL and factors that contribute to HRQOL of female children with $\mathrm{CAH}$ from the perspectives of children with $\mathrm{CAH}$ and their caregiver.

Research Question 4: What themes on HRQOL and factors that may contribute to the HRQOL of children with CAH emerged during interviews with: 


\section{a. children with $\mathrm{CAH}$ ? \\ b. caregivers of children with $\mathrm{CAH}$ ?}

The basis for the aforementioned question is grounded in Bronfenbrenner's ecological perspective in which outcomes are influenced by individual and ecological factors such as process, person, context and time. In the current study, participants provided insights into their perception about HRQOL and highlighted many factors that impact HRQOL including the importance of friends and family, personal characteristics (i.e., the ability to adapt and self-esteem), the environment (i.e., home, school), and the impact of time.

During discussions about living with $\mathrm{CAH}$, both children and caregivers identified friends and family as process factors that contribute to HRQOL. The function of friends and family were related to aspects of social support, the child's welfare (i.e., assisting with care), as well as buffers to stressful life situations. Both children and caregivers believed that friends and family were amongst the most important aspects of HRQOL.

It is important to consider that family and friends may serve as mediators as to how life is viewed and may project their feelings and judgments about the child's disorder. Engberg et al. (2016) found that caregiver and peer support as well as understanding of the disorder were important factors when living with CAH. Therefore, continued support and positive familial and friend relationships throughout childhood and adolescence are essential to children's HRQOL.

Personal characteristics are characterized by children's and caregivers' attitudes and approach to life situations and the consequences thereof, which in turn impacts HRQOL (Wrosch \& Scheier, 2003). In the present study, personal characteristics were traits that define the child's identity and indirectly impact HRQOL. The personal characteristics identified were the child's ability to adapt and psychological well-being.

Adaptation is a process that includes coping strategies such as how children view their situation, handle their emotions, manage behaviors, and act to decrease the causes of stress (Dellenmark-Bloom, Chaplin, Jonsson, Gatzinsky, Quitmann, \& Abrahmsson, 2016). Although not explicitly stated, adaptation in female children and adolescents with CAH was exhibited several ways. Common to all children were adaptive strategies such as being active in sports or extracurricular activities. Both children and caregivers talked about children's skills and the importance of certain activities.

Psychological well-being included the child's attitudes about their disease and the desire to be normal which affected the child's self-esteem and emotional well-being. Most children viewed themselves without illness, and a few even forgot about having $\mathrm{CAH}$, while others were determined to overcome barriers to have a normal life. These findings suggest that positive adaptation and psychological well-being foster positive HRQOL. 
Neither child nor caregiver explicitly discussed contextual factors that may contribute to the HRQOL of children with CAH. However, both children and caregivers reported low school subscale scores within the school aspect of HRQOL. Quitmann et al. (2016) provides an explanation by simply stating that caregivers lack sufficient information regarding their children's social well-being. Verhey and colleagues affirmed that children with conditions like $\mathrm{CAH}$ may desire to keep their internal problems a secret, which means that parents remain unaware of their child's experiences (Verhey et al., 2009). It is equally important to consider children and caregiver's desire to protect each other. Children with CAH may not share their feelings about school with their caregivers because they fear being treated differently (i.e. overprotection) or fear experiencing negative reactions from their caregivers or school personnel (i.e., shock, worry).

Time is a continuum that begins with initial diagnosis and ends when the child and caregiver have successfully learned to adapt to and live with CAH. Adaptation in children with CAH was characterized by an individual's response to the "functional, psychological, and social changes that occur with the onset and experience of living with a chronic illness" (Bishop, 2005, p. 6). In the beginning, caregivers and children commented on the initial period and the period after diagnosis. The initial period was filled with lots of emotions, and often caregivers found it challenging to process the breadth of information provided by healthcare professionals. Many expressed they had some basic knowledge about CAH. However, many admitted they did not fully understand the "specifics." As children grew older, caregivers became more knowledgeable and familiar with the disorder.

\section{Specific Aim Four}

The last aim was to compare the quantitative and qualitative child and caregiver results on HRQOL of female children with CAH.

Research Question 5: To what extent do the themes on HRQOL emerging from interviews with children support the self-reported HRQOL as measured by the KINDL-R?

Research Question 6: To what extent do the themes on HRQOL emerging from interviews with caregivers support the caregiver proxy-reported HRQOL as measured by the KINDL-R?

Research Question 7: To what extent do the themes on HRQOL emerging from interviews with children and their caregivers support the self-report and caregiver proxy-reported HRQOL as measured by the KINDL-R?

Overall, many of the QUAN results were reflected in the QUAL themes, categories, and comments. Many of the discrepant findings were true differences in perspectives. Hence, the developmental challenges of childhood must be considered. 
Younger children's perceptions about their HRQOL change as they enter adolescence. Moreover, adolescence is a time in which children develop a sense of identity, focus on social acceptance and find value in everyday life (Frisen, 2007). The children in this study were largely adolescents and the QUAN and QUAL findings mirror this period. It seems as if the younger children were more positive; whereas the adolescents' perceptions of their HRQOL were more critical. This may be attributed to the increased exposure to social and everyday life pressures (Frisen, 2007). Additionally, the impact of parent's role, well-being, and function must also be considered. The literature has shown that mothers tend to project their own feelings in the evaluation of their child's functioning (Quitmann et al., 2016). Stride and colleagues (2005) observed a relationship in the mother's feelings and how they rated the child's QOL. They noted that mothers who reported their own QOL as poor, also rated the QOL of their child as poor (Stride Christopher, Eiser, \& Eiser, 2005). Goldbeck and Melches (2005) provided similar descriptions. Goldbeck and Melches found that there was a significant correlation between the caregiver's QOL and how the caregiver reported the child's QOL.

\section{Strengths and Limitations}

One of the strengths of the study is that it illuminated individual aspects that contribute to an in-depth understanding of experiences of children with $\mathrm{CAH}$ and their caregivers. More specifically, interviews with children and their caregivers elicited different, yet meaningful information about what the problems are and what problems need to be resolved. The study also elucidates information that children and caregivers want healthcare professionals to know about their experiences and how to improve the lives of children with CAH. The research on HRQOL in female children with CAH is scarce and few studies utilize qualitative and quantitative data from the child and caregiver to understand the HRQOL of female children with CAH. Findings from this study can guide and support future research about the HRQOL of female children with CAH.

Moreover, another strength of the study is the utilization of the KINDL-R. Apart from the psychometric properties of the questionnaire, the KINDL-R takes into consideration the psychological underpinnings of the concepts that describe HRQOL. Many instruments such as the PedsQL, and KIDSCREEN primarily focus on the physical concepts that describe HRQOL. Additionally, many of underpinnings and subscales of the KINDL-R fit the constructs in Bronfrennber's bioecological model of human development.

The study has several limitations. The study included a small sample size. This means that study did not allow for transferability and power to detect sufficient differences in child and caregiver perspectives. Also despite efforts to recruit children and caregivers from all 50 states, the majority of subjects were clustered in one geographical location and not representative of the entire population. There was also a lack of diversity. The child and caregiver sample was primarily Caucasian and overwhelmingly, well-educated. The caregivers in this study may differ from other 
caregivers of children with CAH especially in terms of education. The study is also limited in that it only measured HRQOL at one point in time rather than measuring it over time. An additional limitation was the use of a generic questionnaire rather than one that was validated and disease specific for $\mathrm{CAH}$.

\section{Practice Implications}

Health-related quality of life is multidimensional and focuses on physical, psychological, and social well-being which reinforces the importance of improving comprehensive care. Thus, the traditional "stop" and "go" approach to care is no longer optimal for children with chronic disorders like CAH (Witchel, 2010, p. 1). The findings of this study could shed light on children's and caregiver's needs that are not met by current practice. Children with $\mathrm{CAH}$ and their families needed an environment where they could express concerns, receive compassionate and culturally sensitive care, have readily accessible information, and form partnerships with well-informed clinicians and support groups.

The findings of this study also informed clinicians that children with CAH place emphasis on less observable health problems. It is equally important to understand that children and adolescents strive to be normal so the focus on care should be wellness rather than illness. Nurses and clinicians should include children in discussions about their own care and encourage independence. In doing so, clinicians must make sure that information about $\mathrm{CAH}$ is delivered in a developmentally appropriate way.

Additionally, the findings of this study exposed the potential impact of a common, yet less explored, context on the HRQOL of children with CAH which is school. Children spend many of their waking hours in school and now, more school personnel are supporting children with chronic conditions. However, because CAH is uncommon, many school personnel lack the knowledge and training to adequately support the medical and social needs of children with CAH. This provides clinicians the opportunity to explore better ways to communicate with schools and families as well as opportunities to develop programs to ensure that school personnel and children with CAH feel supported.

The findings of the study also indicate that clinicians should not assume that parents are knowledgeable about their child's condition, are comfortable with disclosing the diagnosis to others, or have the vocabulary to explain the diagnosis to their child, family, or friends. Many caregivers request education that provides information about what CAH means and a "script" to explain the diagnosis to their daughters as well as to others who are involved in the child's care. Based on the findings of the study, clinicians may help children and caregivers develop strategies to navigate talking with others about $\mathrm{CAH}$ and in certain situations talking with others while not revealing too much information. This means that clinicians may help children and caregivers develop ways to communicate with others and enable care or support without identifying the somatic sex disorder (Lundberg et al., 2017). 


\section{Policy Implications}

Implications for public policy involve increasing efforts to allow female children with $\mathrm{CAH}$ to decide on their gender and to be involved in decisions surrounding surgery. Currently, treatment efforts focus largely on maintaining female traits, normalizing female hormones, and helping maintain a feminine appearance. Support and advocacy groups can use this study to educate and encourage children with $\mathrm{CAH}$ and their caregivers to understand that being "normal" does not have to be defined by society's definition of a normal female. More efforts should be geared to support caregivers who choose not to identify and raise their child as a girl and also for those who decide to allow their child to decide on the timing of surgery.

\section{Theoretical Implications}

The use of the BMHD takes into consideration the objective (i.e., age, and gender) and subjective (i.e., relationships and attitudes) developmental constructs as well as individual experiences, feelings and personal beliefs that contribute to children's HRQOL. In this study, there were several similarities between the attributes that make up development and HRQOL, making the model very useful. Although the concepts of HRQOL of children with CAH supports the constructs within the BHMD, future studies should take a greater look into the social/cultural contexts that contribute to the HRQOL of female children with CAH.

This study may be used as an example for other studies that investigate the HRQOL of other populations such as DSD. A longitudinal study that utilizes the BMHD to investigate: 1) the HRQOL at the beginning of diagnosis until the early adulthood; 2) the impact of social contextual factors; and 3) the effects of geographical location and access to support could follow allowing for further understanding of the HRQOL of female children with CAH.

\section{Recommendations for Future Research}

Previous research on the HRQOL of individuals with CAH has primarily focused on adults and psychosexual outcomes; whereas pediatric data that involves the use of QUAN and QUAL is scarce. This study shed light into the many aspects of HRQOL of female children with $\mathrm{CAH}$ while also providing several recommendations for future research. Recommendations involve the use of interdisciplinary, patient centered delivery of care models such as medical homes for children with chronic disorders like CAH. In a medical home delivery model, the focus of care shifts from treatment and management to focus on developmental perspectives and individualized guidance to the meet children's and caregivers' needs (Witchel, 2010).

Furthermore, increasing the sample size and including a more diverse sample would better reflect children with $\mathrm{CAH}$ and caregivers from all ethnicities, social status, 
and backgrounds. It would also increase the generalizability of the study findings and allow the identification of significant and different variables that contribute to the HRQOL of female children with CAH. A research consortium specifically for children and adults with CAH would be invaluable for research and the potential for improving their health and HRQOL.

Additionally, the development and use of validated, disease specific, instruments would allow investigators to truly capture the HRQOL of individuals with CAH and DSDs. The adoption of a theoretical model to guide investigators to make predictions about how individuals will differ and how HRQOL will change over time is needed. Also, a developmental approach that is sensitive and takes into account the specific HRQOL domains instead of ad hoc domains is equally important (Eiser \& Morse, 2001b).

\section{Conclusion}

Using a comprehensive approach offered an understanding of what it is like for children to live with $\mathrm{CAH}$ from the perspective of the child and caregiver. By quantitatively measuring HRQOL and using qualitative interview data, the information in this study may shed light on children's and caregivers' needs that are not met by current guidelines. Clinicians may better support children and families by expanding the focus beyond sex development to include: 1) information about the different ways of knowing (i.e., knowing how to talk with others and with their child); 2) self-management and independence (i.e., learning how to give medication and handling responsibility as children grown older); and 3) psychological support (i.e., coping with a new diagnosis, adapting to the disorder). Expanding the focus has the potential to optimize treatment that will hopefully meet the needs of children with $\mathrm{CAH}$ and their caregivers. This study was the first to use a mixed methods approach to understand the HRQOL and factors that contribute to the HRQOL of female children with CAH. Therefore, this study has contributed to nursing science and science about the HRQOL of children with CAH. Future studies employing mixed methods may aid in the understanding of the effects of treatment, and allow for the development of more sensitive assessment measures of HRQOL. Finally, the information obtained from this study may provide a foundation to further the investigation of HRQOL in female children with $\mathrm{CAH}$, to better support caregivers of children with CAH, and to improve children's overall HRQOL. 


\section{LIST OF REFERENCES}

Allen, D. B., Hoffman, G. L., Fitzpatrick, P., Laessig, R., Maby, S., \& Slyper, A. (1997). Improved precision of newborn screening for congenital adrenal hyperplasia using weight-adjusted criteria for 17-hydroxyprogesterone levels. The Journal of Pediatrics, 130(1), 128-133.

Alpern, A. N., Gardner, M., Kogan, B., Sandberg, D. E., \& Quittner, A. L. (2017). Development of health-related quality of life instruments for young children with disorders of sex development (DSD) and their parents. Journal of Pediatric Psychology, 42(5), 544-558. doi:10.1093/jpepsy/jsw022.

Amaral, R. C., Inacio, M., Brito, V. N., Bachega, T. A., Domenice, S., Arnhold, I. J., . . . Mendonca, B. B. (2015). Quality of life of patients with 46,XX and 46,XY disorders of sex development. Clinical Endocrinology (Oxf), 82(2), 159-164. doi:10.1111/cen.12561.

Arlt, W., Willis, D. S., Wild, S. H., Krone, N., Doherty, E. J., Hahner, S., . . Ross, R. J. (2010). Health status of adults with congenital adrenal hyperplasia: A cohort study of 203 patients. Journal of Clinical Endocrinology \& Metabolism, 95(11), $5110-5121$.

Bachelot, A., Plu-Bureau, G., Thibaud, E., Laborde, K., Pinto, G., Samara, D., . . . Touraine, P. (2007). Long-term outcome of patients with congenital adrenal hyperplasia due to 21-hydroxylase deficiency. Hormone Research in Paediatrics, 67(6), 268-276.

Bandayrel, K., \& Johnston, B. C. (2014). Recent advances in patient and proxy-reported quality of life research. Health and Quality of Life Outcomes, 12(1), 1-9. doi:10.1186/s12955-014-0110-7.

Bennecke, E., Grüters, A., Köhler, B., Thyen, U., \& Lux, A. (2017). Health-related quality of life and psychological well-being in adults with differences/disorders of sex development. Clinical Endocrinology (Oxf), 86(4), 634-643. doi:10.1111/cen.13296.

Berenbaum, S. A., Korman Bryk, K., Duck, S. C., \& Resnick, S. M. (2004). Psychological adjustment in children and adults with congenital adrenal hyperplasia. The Journal of Pediatrics, 144(6), 741-746. doi:http://dx.doi.org/10.1016/j.jpeds.2004.03.037.

Bishop, M. (2005). Quality of life and psychosocial adaptation to chronic illness and disability: preliminary analysis of a conceptual and theoretical synthesis. Rehabilitation Counseling Bulletin, 48(4), 219-253 216p.

Boyse, K. L., Gardner, M., Marvicsin, D. J., \& Sandberg, D. E. (2014). It was an overwhelming thing: parents' needs after infant diagnosis with congenital adrenal hyperplasia. Journal of Pediatric Nursing, 29(5), 436-441. doi:http://dx.doi.org/10.1016/j.pedn.2014.01.007.

Braga, L. H., \& Pippi Salle, J. L. (2009). Congenital adrenal hyperplasia: a critical appraisal of the evolution of feminizing genitoplasty and the controversies surrounding gender reassignment. European Journal of Pediatric Surgery, 19(4), 203-210. 
Bronfenbrenner, U. (1994). Ecological models of human development. International Encyclopedia of Education, 3(2nd), 37-43.

Bronfenbrenner, U. (2004). Making human beings human: bioecological perspectives on human development. The SAGE Program on Applied Developmental Science: SAGE Publications (CA).

Bronfenbrenner, U., \& Morris, P. (2006). The bioecological model of human development. In R. M. Lerner (Ed.), Handbook of Child Psychology: Theoretical Models of Human Development (Vol. 1, pp. 793-828): John Wiley \& Son, Inc.

Bullinger, M., Brutt, A. L., Erhart, M., Ravens-Sieberer, U., \& Group, B. S. (2008). Psychometric properties of the KINDL-R questionnaire: results of the BELLA study. European Child \& Adolescent Psychiatry, 17 Suppl 1, 125-132. doi:10.1007/s00787-008-1014-z.

Bullinger, M., \& Ravens-Sieberer, U. (1995). General principles, methods and areas of application of quality of life research in children. Praxis der Kinderpsychologie und Kinderpsychiatrie, 44(10), 391-399.

Bullinger, M., Schmidt, S., Petersen, C., \& Ravens-Sieberer, U. (2006). Quality of lifeevaluation criteria for children with chronic conditions in medical care. Journal of Public Health, 14(6), 343-355. doi:10.1007/s10389-006-0066-0.

Cantrell, M., \& Kelly, M. (2015). Health-Related Quality of Life for Chronically Ill Children. The American Journal of Maternal Child Nursing, 40(1).

Capio, C. M., Sit, C. H. P., \& Abernethy, B. (2014). Physical well-being. In A. C. Michalos (Ed.), Encyclopedia of Quality of Life and Well-Being Research (pp. 4805-4807). Dordrecht: Springer Netherlands.

Casteras, A., De Silva, P., Rumsby, G., \& Conway, G. S. (2009). Reassessing fecundity in women with classical congenital adrenal hyperplasia $(\mathrm{CAH})$ : normal pregnancy rate but reduced fertility rate. Clinical Endocrinology (Oxf), 70(6), 833-837.

Centers for Disease Control and Prevention (2013). Chronic Disease Prevention and Health Promotion. Retrieved from http://www.cdc.gov/chronicdisease/.

Cicognani, E. (2014). Social well-being. In A. C. Michalos (Ed.), Encyclopedia of Quality of Life and Well-Being Research (pp. 6193-6197). Dordrecht: Springer Netherlands.

Cohen-Bendahan, C. C. C., van de Beek, C., \& Berenbaum, S. A. (2005). Prenatal sex hormone effects on child and adult sex-typed behavior: methods and findings. Neuroscience and Biobehavioral Reviews, 29(2), 353-384.

Connolly, M. A., \& Johnson, J. A. (1999). Measuring quality of life in paediatric patients. PharmacoEconomics, 16(6), 605-625.

Cordeiro, G., Silva, I., Marcos, E., Goulart, A., Jose das Chagas, A., \& Kater, C. (2013). Final height in congenital adrenal hyperplasia: the dilemma of hypercortisolism versus hyperandrogenism. Arquivos Brasileiros De Endocrinologia E Metabologia, 57(2), 126-131.

Cornean, R. E., Hindmarsh, P. C., \& Brook, C. G. D. (1998). Obesity in 21-hydroxylase deficient patients. Archives of Disease in Childhood, 78(3), 261-263.

Crawford, J. M., Warne, G., Grover, S., Southwell, B. R., \& Hutson, J. M. (2009). Results from a pediatric surgical centre justify early intervention in disorders of sex development. Journal of Pediatric Surgery, 44(2), 413-416. 
Creswell, J. W. (2007). Qualitative Inquiry \& Research Design. Choosing among five approaches. Thousand Oaks, CA: Sage.

Creswell, J. (2014). A Concise Introduction to Mixed Methods Research. Thousand Oaks, CA: SAGE.

Cull, M. L. (2005). A support group's perspective. BMJ : British Medical Journal, 330(7487), 341-341. doi:10.1136/bmj.38365.883843.79.

Dellenmark-Blom, M., Chaplin, J. E., Jönsson, L., Gatzinsky, V., Quitmann, J. H., \& Abrahamsson, K. (2016). Coping strategies used by children and adolescents born with esophageal atresia - a focus group study obtaining the child and parent perspective. Child: Care, Health and Development, 42(5), 759-767. doi:10.1111/cch.12372.

Demirci, C., \& Witchel, S. F. (2008). Congenital adrenal hyperplasia. Dematologic Therapy, 21(2), 340-353.

Earle, V. (2010). Phenomenology as research method or substantive metaphysics? An overview of phenomenology's uses in nursing. Nursing Philosophy, 11(4), 286296. doi:10.1111/j.1466-769X.2010.00458.x.

Ediati, A., Faradz, S. M. H., Juniarto, A. Z., van der Ende, J., Drop, S. L. S., \& Dessens, A. B. (2015). Emotional and behavioral problems in late-identified Indonesian patients with disorders of sex development. Journal of Psychosomatic Research, 79(1), 76-84. doi:https://doi.org/10.1016/j.jpsychores.2014.12.007.

Eiser, C., \& Morse, R. (2001a). Quality-of-life measures in chronic diseases of childhood. Health Technology Assessment, 5(4), 156. doi:10.3310/hta5040.

Eiser, C., \& Morse, R. (2001b). Can parents rate their child's health-related quality of life? Results of a systematic review. Quality of Life Research, 10. doi:10.1023/A:1012253723272.

Eiser, C., \& Varni, J. W. (2013). Health-related quality of life and symptom reporting: similarities and differences between children and their parents. European Journal of Pediatrics, 172(10), 1299-1304. doi:10.1007/s00431-013-2049-9.

Engberg, H., Frisén, L., Möller, A., Hagenfeldt, K., \& Nordenskjöld, A. (2016). The experience of women living with congenital adrenal hyperplasia: Impact of the condition and the care given. Clinicial Endocrinology (Oxf), 85(1), 21-28. doi:10.1111/cen.13054.

Erhart, M., Ellert, U., Kurth, B. M., \& Ravens-Sieberer, U. (2009). Measuring adolescents' HRQOL via self-reports and parent proxy reports: an evaluation of the psychometric properties of both versions of the KINDL-R instrument. Health and Quality of Life Outcomes, 7(77), 1-2.

Eugster, E. A., DiMeglio, L. A., Wright, J. C., Freidenberg, G. R., Seshadri, R., \& Pescovitz, O. H. (2001). Height outcome in congenital adrenal hyperplasia caused by 21-hydroxylase deficiency: A meta-analysis. The Journal Of Pediatrics, 138(1), 26-32. doi:http://dx.doi.org/10.1067/mpd.2001.110527.

Fagerholm, R., Taskinen, S., Mattila, A. K., Roine, R. P., \& Sintonen, H. (2012). Mental health and quality of life after feminizing genitoplasty. Journal of Pediatric Surgery, 47(4), 747-751. doi:10.1016/j.jpedsurg.2011.08.018.

Ferrans, C. E., Zerwic, J. J., Wilbur, J. E., \& Larson, J. L. (2005). Conceptual model of health-related quality of life. Journal of Nursing Scholarship, 37(4), 336-342. doi:10.1111/j.1547-5069.2005.00058.x. 
Frisén, A. (2007). Measuring health-related quality of life in adolescence. Acta Paediatrica, 96(7), 963.

Frisen, L., Nordenstrom, A., Falhammar, H., Filipsson, H., Holmdahl, G., Janson, P. O., . . . Nordenskjold, A. (2009). Gender role behavior, sexuality, and psychosocial adaptation in women with congenital adrenal hyperplasia due to CYP21A2 deficiency. Journal of Clinical Endocrinology \& Metabolism, 94(9), 3432-3439. doi:10.1210/jc.2009-0636.

Garland, J., \& Diamond, M. (2014). Evidence regarding cosmetic and medically unnecessary surgery on infants. Journal of Pediatric Urology, 10(1), 2-6.

Gaspar, T., Ribeiro, J. P., de Matos, M. G., Leal, I., \& Ferreira, A. (2012). Health-related quality of life in children and adolescents: Subjective well being. Spanish Journal of Psychology, 15(1), 177-186.

Goldbeck, L. \& Melches, J. (2005). Quality of life in families of children with congenital heart disease. Quality of Life Research(8), 1915.

Gilban, D. L. S., Alves Junior, P. A. G., \& Beserra, I. C. R. (2014). Health related quality of life of children and adolescents with congenital adrenal hyperplasia in Brazil.

Health and Quality of Life Outcomes, 12(107), 1-9. doi:10.1186/s12955-0140107-2.

Gunnarsson, C., Ryan, M. P., Marelli, C., Baker, E. R., Stewart, P. M., Johannsson, G., \& Biller, B. M. K. (2017). Healthcare burden in patients with adrenal insufficiency. Journal of the Endocrine Society, 1(5), 512-523. doi:10.1210/js.2016-1064.

Han, T. S., Walker, B. R., Arlt, W., \& Ross, R. J. (2014). Treatment and health outcomes in adults with congenital adrenal hyperplasia. Nature Reviews Endocrinology, 10(2), 115-124.

Hines, M., Brook, C., \& Conway, G. S. (2004). Androgen and psychosexual development: care gender identity, sexual orientation, and recalled childhood gender role behavior. The Journal of Sex Research, 41(1), 75-81.

Idris, A. N., Chandran, V., Syed Zakaria, S. Z., \& Rasat, R. (2014). Behavioural outcome in children with congenital adrenal hyperplasia: Experience of a single centre. International Journal of Endocrinology, 2014, 9. doi:10.1155/2014/483718.

Jääskeläinen, J., Voutilainen, R., Hippeläinen, M., \& Kiekara, O. (2000). Child rate, pregnancy outcome and ovarian function in females with classical 21-hydroxylase deficiency. Acta Obstetricia et Gynecologica Scandinavica, 79(8), 687-692. doi:10.1034/j.1600-0412.2000.079008687.x.

Johnson, T., Kaplan, L., \& Ouyang, P, et.al. (2013). National Institutes of Health evidence-based methodology workshop on polycystic ovary syndrome (PCOS) p. 1. Retrieved from https://prevention.nih.gov/programs-events/pathways-toprevention/previous-workshops/pcos/workshop-resources.

Jokovic, A., Locker, D., \& Guyatt, G. (2004). How well do parents know their children? Implications for proxy reporting of child health-related quality of life. Quality $O f$ Life Research: An International Journal Of Quality Of Life Aspects Of Treatment, Care And Rehabilitation, 13(7), 1297-1307.

Jürgensen, M., Kleinemeier, E., Kropf, S., Lux, A., \& Thyen, U. (2009). Clinical evaluation study of the German network of disorders of sex development (DSD)/intersexuality: study design, description of the study population, and data 
quality. BMC Public Health, Vol 9, Iss 1, p 110 (2009)(1), 110. doi:10.1186/14712458-9-110.

Jurgensen, M., Lux, A., Wien, S., Kleinnemeier, E., Hiort, O., \& Thyen, U. (2014). Health-related quality of life in children with disorders of sex development (DSD) .European Journal of Pediatrics, 173, 893-903. doi:10.1007/s0043-014-2264-z.

Keyes, C. M. (2002). The mental health continuum: From languishing to flourishing in life. Journal of Health and Social Behavior, (2), 207.

Kim, M., Dao-Tran, A., Davidowitz, E., Tseng, T., Gilsanz, V., Ryabets-Lienhard, A., Nguyen, E., \& Geffner, M. E. (2016). Carotid intima-media thickness is associated with increased androgens in adolescents and young adults with classical congenital adrenal hyperplasia. Hormone Research in Paediatrics, 85(4), 242-249. doi:10.1159/000444169.

Knorr, D., \& Hinrichsen de Lienau, S. G. (1988). Persistent obesity and short final height after corticoid overtreatment for congenital adrenal hyperplasia (CAH) in infancy. Acta Paediatrica Japonica; Overseas Edition, 30 Suppl, 89-92.

Kochar, I. P. S., \& Jindal, R. (2011). Diagnosis and management of congenital adrenal hyperplasia in the child and adolescent. Apollo Medicine, 8(4), 261-265. doi:http://dx.doi.org/10.1016/S0976-0016(11)60002-1.

Koot, H. (2014). Quality of life in child and adolescent illness: Concepts, methods and findings: Routledge.

Lantos, J. D. (2013). The battle lines of sexual politics and medical morality. Hastings Center Report, 43(2), 3-4. doi:10.1002/hast.147.

Lin-Su, K., Nimkarn, S., \& New, M. I. (2008). Congenital adrenal hyperplasia in adolescents: diagnosis and management. Annals of the New York Academy of Sciences, 1135, 95-98. doi:10.1196/annals.1429.021.

Lundberg, T., Roen, K., Lindstrom, A., \& Hegarty, P. (2017). From knowing nothing to knowing what, how and now: Parents' experiences of caring for their children with congenital adrenal hyperplasia. Journal of Pediatric Psychology, 42(5), 520529. doi:10.1093/jpepsy/jsw001.

Malouf, M. A., Inman, A. G., Carr, A. G., Franco, J., \& Brooks, L. M. (2010). Healthrelated quality of life, mental health and psychotherapeutic considerations for women diagnosed with a disorder of sexual development: congenital adrenal hyperplasia. International Journal of Endocrinology, 2010, 1-11. doi:10.1155/2010/253465.

Matza, L. S., Swensen, A. R., Flood, E. M., Secnik, K., \& Leidy, N. K. (2004). Assessment of health-related quality of life in children: A review of conceptual, methodological, and regulatory issues. Value in Health, 7(1), 79-92. doi:10.1111/j.1524-4733.2004.71273.x.

McGuire, C., \& Chadwick, G. (2012). Protecting study volunteers in research: A manual for investigative sites. Boston, MA: CenterWatch.

Merke, D. P., \& Poppas, D. P. (2013). Management of adolescents with congenital adrenal hyperplasia. The Lancet. Diabetes \& Endocrinology, 1(4), 341-352. doi:10.1016/S2213-8587(13)70138-4.

Mnif, M., Kamoun, M., Kacem, F., Mnif, F., Charfi, N., Naceur, B., . . A Abid, M. (2013). Reproductive outcomes of female patients with congenital adrenal hyperplasia 
due to 21-hydroxylase defi ciency. Indian Journal of Endocrinology and Metabolism, 17(5), 790-793. doi:10.4103/2230-8210.117196.

Mnif, M. F., Kamoun, M., Mnif, F., Charfi, N., Kallel, N., Naceur, B. B., . . Keskes, L. A. (2012). Long-term outcome of patients with congenital adrenal hyperplasia due to 21-hydroxylase deficiency. American Journal of the Medical Sciences, 344(5), 363-373. doi:10.1097/MAJ.0b013e31824369e4.

Mooij, C. F., Kroese, J. M., Claahsen-van der Grinten, H. L., Tack, C. J., \& Hermus, A. R. (2010). Unfavourable trends in cardiovascular and metabolic risk in paediatric and adult patients with congenital adrenal hyperplasia? Clinical Endocrinology (Oxf), 73(2), 137-146. doi:10.1111/j.1365-2265.2009.03690.x.

Morse, J., \& Neihaus, L. (2009). Mixed Method Design: Who Needs It? Mixed Methods Design: Principles and Procedures. Walnut, Creek: CA: Left Coast Press.

National Alliance for Caregiving (2015). Caregiving in the U.S.

Nermoen, I., Husebye, E. S., Svartberg, J., \& Lovas, K. (2010). Subjective health status in men and women with congenital adrenal hyperplasia: a population-based survey in Norway. European Journal of Endocrinology, 163(3), 453-459.

New, M. (2004). An update of congenital adrenal hyperplasia. Annals of the New York Academy of Sciences, 1038, 14-43. doi:10.1196/annals.1315.009.

New, M. (2006). Extensive clinical experience: nonclassical 21-hydroxylase deficiency. The Journal of Clinical Endocrinology and Metabolism, 91(11), 4205-4214.

Nimkarn, S., Lin-Su, K., \& New, M. (2011). Steroid 21-hydroxylase deficiency congenital adrenal hyperplasia. Pediatric Clinics of North America, 58, 12811300 .

Nimkarn, S., \& New, M. I. (2009). Prenatal diagnosis and treatment of congenital adrenal hyperplasia due to 21-hydroxylase deficiency. Molecular and Cellular Endocrinology, 300(1-2), 192-196. doi:http://dx.doi.org/10.1016/j.mce.2008.11.027

Nordenskjold, A., Holmdahl, G., Frisen, L., Falhammar, H., Filipsson, H., Thoren, M., . . .Hagenfeldt, K. (2008). Type of mutation and surgical procedure affect long-term quality of life for women with congenital adrenal hyperplasia. Journal of Clinical Endocrinology \& Metabolism, 93(2), 380-386.

Nordenstrom, A. (2011). Adult women with 21-hydroxylase deficient congenital adrenal hyperplasia, surgical and psychological aspects. Current Opinions in Pediatrics, 23(4), 436-442. doi:10.1097/MOP.0b013e32834810a4.

Olson AL, Seidler AB, Goodman D, Gaelic S, Nordgren R. (2004). School professionals' perceptions about the impact of chronic illness in the classroom. Archives of Pediatrics \& Adolescent Medicine. 158(1):53-58. doi:10.1001/archpedi.158.1.53.

Pasterski, V., Zucker, K. J., Hindmarsh, P. C., Hughes, I. A., Acerini, C., Spencer, D., . . . Hines, M. (2015). Increased cross-gender identification independent of gender role behavior in girls with congenital adrenal hyperplasia: Results from a standardized assessment of 4- to 11-year-old children. Archives of Sexual Behavior, 44(5), 1363-1375. doi:10.1007/s10508-014-0385-0.

Pickard, A. S., \& Knight, S. J. (2005). Proxy evaluation of health-related quality of life: a conceptual framework for understanding multiple proxy perspectives. Medical Care, (5), 493. 
Plat, L., Leproult, R., L'Hermite-Baleriaux, M., Fery, F., Mockel, J., Polonsky, K. S., \& Van Cauter, E. (1999). Metabolic effects of short-term elevations of plasma cortisol are more pronounced in the evening than in the morning. Journal of Clinical Endocrinology \& Metabolism, 84(9).

Polit, D. F., \& Beck, C. T. (2008). Philadelphia: PA: Lippincott Williams \& Wilkins.

Quintos, J., Vogiatzi, M., Harbison, M. D., \& New, M. (2000). Growth hormone therapy alone or in combination with to improve height deficit in CAH. The Journal of Clinical Endocrinology and Metabolism, 86(4), 1511-1516.

Quitmann, J., Rohenkohl, A., Sommer, R., Bullinger, M., \& Silva, N. (2016). Explaining parent-child disagreement in generic and short stature-specific health-related quality of life reports: do family and social relationships matter? Health and Quality of Life Outcomes, 14, 150. doi:10.1186/s12955-016-0553-0.

Rajmil, L., Herdman, M., Fernandez de Sanmamed, M.-J., Detmar, S., Bruil, J., RavensSieberer, U., . . . Auquier, P. (2004). Generic health-related quality of life instruments in children and adolescents: a qualitative analysis of content. Journal of Adolescent Health, 34(1), 37-45. doi:http://dx.doi.org/10.1016/S1054139X(03)00249-0.

Ravens-Sieberer, U., \& Bullinger, M. (2000). KINDL-R Questionnaire for measuring health-related quality of life in children and adolescents manual (Revised Version). 1-25.

Ravens-Sieberer, U., Erhart, M., Wille, N., Wetzel, R., Nickel, J., \& Bullinger, M. (2006). Generic health-related quality-of-life assessment in children and adolescents. PharmacoEconomics, 24(12), 1199-1220. doi:10.2165/00019053200624120-00005.

Ravens-Sieberer, U., Erhart, M., Wille, N., Bullinger, M., \& the Bella Study Group. (2008). Health-related quality of life in children and adolescents in Germany: results of the BELLA study. European Child Adolescent Psychiatry, 17 Suppl 1, 148-156. doi:10.1007/s00787-008-1016-x.

Ravens-Sieberer, U., Gosch, A., Abel, T., Auquier, P., Bellach, B. M., Bruil, J., . . . Rajmil, L. (2001). Quality of life in children and adolescents: a European public health perspective. Sozial- Und Präventivmedizin, 46(5), 294-302.

Reichman, D. E., White, P. C., New, M. I., \& Rosenwaks, Z. (2014). Views and reviews: Fertility in patients with congenital adrenal hyperplasia. Fertility and Sterility, 101, 301-309. doi:10.1016/j.fertnstert.2013.11.002.

Riepe, F. G., \& Sippell, W. G. (2007). Recent advances in diagnosis, treatment, and outcome of congenital adrenal hyperplasia due to 21-hydroxylase deficiency. Reviews in Endocrinology and Metabolic Disorders, 8(4), 349-363. doi:10.1007/s11154-007-9053-1.

Reisch, N., Arlt, W., \& Krone, N. (2011). Health problems in congenital adrenal hyperplasia due to 21-hydroxylase deficiency. Hormone Research in Paediatrics, 76(2), 73-85.

Reisch, N., Hahner, S., Bleicken, B., Flade, L., Pedrosa Gil, F., Loeffler, M., . . . Quinkler, M. (2011b). Quality of life is less impaired in adults with congenital adrenal hyperplasia because of 21-hydroxylase deficiency than in patients with primary adrenal insufficiency. 74(2), 166-173. doi:10.1111/j.1365-

2265.2010.03920.x. 
Rosa, E. M., \& Tudge, J. (2013). Urie Bronfenbrenner's theory of human development: Its evolution from ecology to bioecology. Journal of Family Theory \& Review, 5(4), 243-258. doi:10.1111/jftr.12022.

Sandberg, D.E., \& Mazur, T. (2014). A noncategorical approach to the psychosocial care of persons with DSD and their families. In B.P.C Kreukels, T.D. Steensma, \& A.L.C. de Vries (Eds.), Gender dysphoria and disorders of sex development. 93114.New York, NY: Springer.

Sandberg David, E., Robbins Natashia, M., Litvak, M., \& Stout Stephanie, A. (2010). Congenital adrenal hyperplasia: Classification of studies employing psychological endpoints. International Journal of Pediatric Endocrinology, Vol 2010, Iss 1, p 191520 (2010)(1), 191520. doi:10.1186/1687-9856-2010-191520.

Schober, J., Nordenstrom, A., Hoebeke, P., Lee, P., Houk, C., Looijenga, L., . . . Woodhouse, C. (2012). Disorders of sex development: Summaries of long-term outcome studies. Journal of Pediatric Urology, 8(6), 616-623.

Speiser, P. W., Azziz, R., Baskin, L. S., Ghizzoni, L., Hensle, T. W., Merke, D. P., . . White, P. C. (2010). Congenital adrenal hyperplasia due to steroid 21-hydroxylase deficiency: An endocrine society clinical practice guideline. The Journal of Clinical Endocrinology and Metabolism, 95(9), 4133-4160. doi:10.1210/jc.20092631.

Strandqvist, A., Falhammar, H., Lichtenstein, P., Hirschberg, A. L., Wedell, A., Norrby, C., . . . Nordenström, A. (2014). Suboptimal psychosocial outcomes in patients with congenital adrenal hyperplasia: epidemiological studies in a nonbiased national cohort in sweden. Journal of Clinical Endocrinology \& Metabolism, 99(4), 1425-1432 1428p. doi:10.1210/jc.2013-3326.

Stride Christopher, B., Eiser, J. R., \& Eiser, C. (2005). Quality of life in children newly diagnosed with cancer and their mothers. Health and Quality of Life Outcomes, Vol 3, Iss 1, p 29 (2005)(1), 29. doi:10.1186/1477-7525-3-29.

Subbarayan, A., Dattani, M. T., Peters, C. J., \& Hindmarsh, P. C. (2014). Cardiovascular risk factors in children and adolescents with congenital adrenal hyperplasia due to 21-hydroxylase deficiency. Clinical Endocrinology (Oxf), 80(4), 471-477. doi:10.1111/cen.12265.

Taylor, R. M., Gibson, F., \& Franck, L. S. (2008a). A concept analysis of health-related quality of life in young people with chronic illness. Journal of Clinical Nursing, 17(14), 1823-1833. doi:10.1111/j.1365-2702.2008.02379.x.

Taylor, R. M., Gibson, F., \& Franck, L. S. (2008b). The experience of living with a chronic illness during adolescence: A critical review of the literature. Journal of Clinical Nursing, 17(23), 3083-3091. doi:10.1111/j.1365-2702.2008.02629.x.

Turcu, A. F., \& Auchus, R. J. (2015). The next 150 years of congenital adrenal hyperplasia. The Journal Of Steroid Biochemistry And Molecular Biology, 153, 63-71. doi:10.1016/j.jsbmb.2015.05.01.

Tudge, J., Mokrova, I., Hatfield, B., \& Karnik, R. (2009). Uses and Misuses of Bronfenbrenner's bioecological theory of human development. Journal of Family Theory \& Review, 1, 198-210.

University of Tennessee Health Science Center Institutional Review Board. (2017). Standard Operating Procedure: UTHSC IRB Additional Protections: Children, Version Number 012. Retrieved from 
https://www.uthsc.edu/research/compliance/irb/researchers/documents/additionalprotections-for-children.pdf.

Upton, P., Lawford, J., \& Eiser, C. (2008). Parent-child agreement across child healthrelated quality of life instruments: A review of the literature. Quality of Life Research, (6), 895. doi:10.1007/s11136-008-9350-5.

Van Manen, M. (1990). Researching lived experience : human science for an action sensitive pedagogy. State University of New York Press. Albany, N.Y..

Verhey, L. H., Kulik, D. M., Ronen, G. M., Rosenbaum, P., Lach, L., \& Streiner, D. L. (2009). Quality of life in childhood epilepsy: What is the level of agreement between youth and their parents? Epilepsy \& Behavior, 14(2), 407-410. doi:http://dx.doi.org/10.1016/j.yebeh.2008.12.008.

Völkl, T. M. K., Simm, D., Beier, C., \& Dörr, H. G. (2006). Obesity among children and adolescents with classic congenital adrenal hyperplasia due to 21-hydroxylase deficiency. Pediatrics, 117(1), e98-e105.

Votava, F., D, T., J, K., Moslinger, D., Baumgartner-Parzer, S. M., Sm, B.-P., . . . Waldhauser, F. (2005). Estimation of the false-negative rate in newborn screening for congenital adrenal hyperplasia. European Journal of Endocrinology,152(2), 869-874.

Wang, C., \& Tian, Q. (2015). The investigation of quality of life in 87 Chinese patients with disorders of sex development. BioMed Research International, 2015, 1-6. doi: $10.1155 / 2015 / 342420$.

White, P. C., \& Speiser, P. W. (2000). Congenital adrenal hyperplasia due to 21hydroxylase deficiency. Endocrine Reviews, 21(3), 245-291.

Wilson, I. B., \& Cleary, P. D. (1995). Linking clinical variables with health-related quality of life: A conceptual model of patient outcomes. JAMA, 273(1), 59-65. doi:10.1001/jama.1995.03520250075037.

Witchel, S. F. (2010). The medical home concept and congenital adrenal hyperplasia: A comfortable habitat! International Journal of Pediatric Endocrinology, 2010(1), 561526. doi:10.1155/2010/561526.

Witchel, S. F., \& Azziz, R. (2011). Congenital adrenal hyperplasia. Journal of Pediatric Adolescent Gynecology, 24(3), 116-126. doi:10.1016/j.jpag.2010.10.001.

World Health Organization (WHO) (2005). The World Health Organization quality of life assessment (WHOQOL): position paper form the World Health Organization. Social Science \& Medicine, 41(10), 1403-1409.

Wrosch, C., \& Scheier, M. F. (2003). Personality and quality of life: The importance of optimism and goal adjustment. Quality of Life Research, 12(1), 59-72. doi:10.1023/A:1023529606137.

Yau, M., Vogiatzi, M., Lewkowitz-Shpuntoff, A., Nimkarn, S., \& Lin-Su, K. (2015). Health-related quality of life in children with congenital adrenal hyperplasia. Hormone Research in Paediatrics, 84(3), 165-171. doi:10.1159/000435855.

Zainuddin, A. A., Grover, S. R., Shamsuddin, K., \& Mahdy, Z. A. (2013). Research on quality of life in female patients with congenital adrenal hyperplasia and issues in developing nations. Journal of Pediatric Adolescent Gynecology, 26(6), 296-304. doi:10.1016/j.jpag.2012.08. 004. 


\title{
APPENDIX A. APPROVED CONSENT FORM FOR CHILDREN WITH CAH
}

\author{
Main Consent Form

TITLE: Health-Related Quality of Life in Female Children with Congenital Adrenal Hyperplasia: A Mixed Methods Study

PRINCIPAL INVESTIGATOR: $\quad$ Carolyn Graff RN, PhD

Boiling Center

711 Jefferson \#182

Memphis TN, 38105

CO-INVESTIGATOR(S): $\quad$ Lacretia Carroll RN, MSN

UTHSC CON- Nursing Academic Programs

$5^{\text {th }}$ FL 920 Madison Building

920 Madison Ave, Memphis TN, 38163-2275

\section{INTRODUCTION:}

A person who takes part in a research study is called a research or study subject. In this consent form "you" refers to the research subject and/or the legally authorized representative.

You are being given the opportunity to participate in this research study. The purpose of this consent form is to help you decide if you want to be in the research study. This consent form may contain words that you do not understand. Please ask the study staff to explain any words or information that you do not clearly understand. We encourage you to talk with your family and friends before you decide to take part in this research study. Please tell the study staff if you are taking part in another research study.

The purpose of this study is to examine the health related quality of life in female children with congenital adrenal hyperplasia.

Approximately 75 pairs of people will be accrued in this study. Fifteen pairs will be participating locally and the other pairs will be accrued nationally. The study will take place at places that can be easily accessed and that are as private as possible, such as a private office, conference room, private group-study room or conference room of a local university library (i.e., University of Tennessee Health Science Center College of Nursing, or Lebonheur Children's Hospital, or the Cares Foundation). The PI will also travel to where the participant(s) is located to conduct study procedures.

Your participation in this study will last no more than 2 hours.

June 2, 2016

Page 1 of 8

Subject or Parent/Legally Authorized Representative Initials 
Main Consent Form

\section{PROCEDURES TO BE FOLLOWED}

Questionnaires and interviews: ( 2 hours or less)

- The researcher will ask that you and your caregiver or the child with congenital adrenal hyperplasia sit separately to answer questions.

- You will sit close to your caregiver or the child, but you will sit where the other person cannot hear what you are saying.

- Information such as your age, the child's medical history (i.e., diagnosis, medications), and information about the child's health will be obtained from you and/or your caregiver.

- Information such as age, gender, ethnicity, education, marital status, place of residence, and employment status will be obtained from the parent/caregiver.

- You and your caregiver will complete a questionnaire called the KINDL-R that asks questions about your health and how your health affects your life.

- You and your caregiver will answer questions about your health and about how your health affects you in separate interviews that will be audio recorded.

\section{RISKS ASSOCIATED WITH PARTICIPATION:}

There is a minimal risk talking to the principal investigator. Some questions may be private and make you feel uncomfortable, but they are important questions that help us to understand how congenital adrenal hyperplasia affects your child's life. All information that is shared will be used for research purposes only, and will only be available to study staff. There is the potential risk of loss of confidentiality. Every effort will be made to keep your information confidential; however, this cannot be guaranteed.

The research may involve risks to you which are currently unforeseeable. You will be told about any new information that might change your decision to be in this study. You may be asked to sign a new consent form if this occurs.

Questionnaires and Interviews

Completion of the interview and KINDL-R Questionnaire may make you feel uncomfortable or cause troublesome feelings or emotions. Questions about your age, gender, ethnicity, marital status, level of education, employment status, and where you live may also make you feel uncomfortable or cause troublesome feelings or emotions. You may refuse to answer any of the questions and you may take a break at any time during the study.

June 2, 2016

Page 2 of 8

Subject or Parent/Legally Authorized Representative Initials

IRB NUMBER: 16-04531-XP

IRB APPROVAL DATE: 06/06/2016

IRB EXPIRATION DATE: 04/14/2017 
Audio Recording:

Having your voice recorded may make you feel uncomfortable. You may take a break during any time of the study. There is also a potential risk of loss of confidentiality that someone who listens to your audio recording might identify you.

\section{BENEFITS ASSOCIATED WITH PARTICIPATION:}

You will not receive any benefits from being in this study. The results of this study may help people with congenital adrenal hyperplasia in the future and benefit society by increasing knowledge about the health related quality of life of children with congenital adrenal hyperplasia.

\section{ALTERNATIVES TO PARTICIPATION:}

You do not have to participate in this study.

You will not have to undergo the following procedures if you do not take part in this study:

- Consent Process

- Have information such as your age, and medical history extracted from your medical record

- Complete the KINDL-R questionnaire

- Complete the audio-recorded interview

- Have information such as your age, and medical history as well as information about your health obtained from you and/or your caregiver.

\section{CONFIDENTIALITY:}

\section{Research records/specimens}

All your paper research records will be stored in locked file cabinets and will be accessible only to research personnel and those entities named below in this section, except as required by law (such as reports of child abuse, plans to commit suicide, etc.).

All your electronic research records will be computer password protected and accessible only to research personnel and those entities named below in this section, except as required by law (such as reports of child abuse, plans to commit suicide, etc.).

\section{Medical Records}

Information about your participation in this study or the results of procedures performed in this study will not be placed in your medical record. 


\section{Main Consent Form}

\section{Presentations/Publications}

While individual details about your case might be provided in publications or presentations about this research, they will not be discussed in a way that would allow you to be individually

identified as a participant.

\section{Authorization to Use and Disclose Information for Research Purposes}

Under federal privacy regulations, you have the right to decide who can review and copy your personal health information (called "protected health information" or PHI). PHI collected in this study may include information such as:

- Past and present medical records

- Records about your study visits

- Records about phone calls made as part of this research

- Research records

By signing this consent form, you are giving your permission for the study staff and the study staff at the University of Tennessee to get your PHI from your doctor and/or facilities where you have received health care. They may also share your PHI with:

- The Institutional Review Board (IRB) at the University of Tennessee Health Science Center

- UT Le Bonheur Pediatric Specialists, Inc

- Researchers at the CARES Foundation

- Le Bonheur Children's Hospital

However, some of these organizations or institutions above do not have the same obligations to protect your PHI.

Your PHI will only be used and/or given to others:

- To do the research

- To study the results

- To see if the research was done correctly

Your PHI will be used until the study is completed.

You may withdraw or take away your permission to use and disclose your PHI at any time. You do this by sending written notice to the study staff. If you withdraw your permission, you may not be able to stay in the study.

When you withdraw your permission, no new PHI will be gathered after that date. However, information that has already been gathered may still be used and given to others. The federal regulations allow you to review or copy your PHI that is used in this study.

June 2, 2016

Page 4 of 8

Subject or Parent/Legally Authorized Representative Initials

IRB NUMBER: 16-04531-XP

IRB APPROVAL DATE: 06/06/2016

IRB EXPIRATION DATE: 04/14/2017 
Main Consent Form

Certificate of Confidentiality

To help us further protect your privacy, the investigators have obtained a Confidentiality Certificate from the Department of Health and Human Services (DHHS).

With this certificate, the investigators cannot be forced (for example, by court subpoena) to disclose research information that may identify you in any Federal, State, or local civil, criminal, administrative, legislative, or other proceedings. Disclosure will be necessary, however, upon request of DHHS for audit or program evaluation purposes.

The Confidentiality Certificate does not prevent you or a member of your family from voluntarily releasing information about yourself or your involvement in this research. Note, however, that if an insurer or employer learns about your participation and obtains your consent to receive research information, then the investigator may not use the Certificate of Confidentiality to withhold this information. This means that you and your family must also actively protect your own privacy.

Finally, the investigator is not prevented from taking steps, including disclosure of your research information to authorities, in order to prevent serious harm to yourself or others.

\section{COMPENSATION AND TREATMENT FOR INJURY:}

You are not waiving any legal rights or releasing the University of Tennessee, Le Bonheur Children's Hospital, UT Le Bonheur Pediatric Specialists, Inc., or the agents of either, from liability for negligence. In the event of physical injury resulting from research procedures, the University of Tennessee, Le Bonheur Children's Hospital, and UT Le Bonheur Pediatric Specialists, Inc., do not have funds budgeted for compensation for medical treatment. Therefore, the University of Tennessee, Le Bonheur Children's Hospital, and UT Le Bonheur Pediatric Specialists, Inc. do not provide for treatment or reimbursement for such injuries.

If you are injured or get sick as a result of being in this study, call the study staff immediately. The study staff will refer you to appropriate health care facilities.

If you are injured or get sick as a result of being in this study, you and/or your insurance will be billed for the costs associated with this medical treatment. No compensation will be available to you for any extra expenses that you may have as the result of research related physical injuries, such as additional hospital bills, lost wages, travel expenses, etc. No compensation will be available to you for any non-physical injuries that you may have as a result of research participation, such as legal problems, problems with your finances or job, or damage to your reputation.

June 2, 2016

Page 5 of 8

Subject or Parent/Legally Authorized Representative Initials

IRB NUMBER: 16-04531-XP

IRB APPROVAL DATE: 06/06/2016

IRB EXPIRATION DATE: 04/14/2017 


\section{QUESTIONS:}

Contact Lacretia Carroll at 901-232-1682 if you have questions about your participation in this study, or if you have questions, concerns, or complaints about the research.

If you feel you have had a research-related injury contact Lacretia Carroll at 901-232-1682 which is 24 -hour/7day telephone number.

You may contact Terrence F. Ackerman, Ph.D., UTHSC IRB Chairman, at 901-448-4824, or visit the IRB website at

http://www.uthsc.edu/research/research_compliance/IRB/participant_complaint.php if you have any questions about your rights as a research subject, or if you have questions, concerns, or complaints about the research.

\section{PAYMENT FOR PARTICIPATION:}

You will receive a $\$ 20$ gift card to a retail store at the completion of each study visit. If you complete all the study visits, you will receive a total of 1 gift card worth $\$ 20$. If you are 12 years and older, the gift card will be given to you; however, if you are under 12 years old, the gift card will be given to your parents for your use. If you do not complete the study, you will be paid for the visits you have completed.

\section{COSTS OF PARTICIPATION:}

There are no costs to you for participating in this study.

\section{VOLUNTARY PARTICIPATION AND WITHDRAWAL:}

Your participation in this research study is voluntary. You may decide not to participate or you may leave the study at any time. Your decision will not result in any penalty or loss of benefits to which you are entitled.

If you decide to stop being part of the study, you should tell your study staff, and any information that you have already provided will be kept in a confidential manner. 


\section{CONSENT OF SUBJECT:}

You have read or have had read to you a description of the research study as outlined above. The investigator or his/her representative has explained the study to you and has answered all the questions you have at this time. You knowingly and freely choose to participate in the study. A copy of this consent form will be given to you for your records.

\begin{tabular}{|c|c|c|}
\hline Signature of Research Subject (18 years +) & Date & Time \\
\hline \multicolumn{3}{|l|}{ Printed Name of Adult Research Subject } \\
\hline Assent of Minor (Ages 14-17) & Date & Time \\
\hline \multicolumn{3}{|l|}{ Printed Name of Minor Research Subject } \\
\hline Signature of Legally Authorized Representative & Date & Time \\
\hline \multicolumn{3}{|l|}{$\overline{\text { Printed Name of Legally Authorized Representative }}$} \\
\hline \multicolumn{3}{|l|}{ Relationship of Legally Authorized Representative } \\
\hline Signature of Person Obtaining Consent & Date & Time \\
\hline
\end{tabular}

In my judgment, the subject and/or the legally authorized representative has voluntarily and knowingly given informed consent and possesses the legal capacity to give informed consent to participate in this research study.

$\begin{array}{lll}\text { Signature of Investigator } & \overline{\text { Date }} \quad \overline{\text { Time }}\end{array}$




\section{Assent Discussion for Subjects 8-13 Years of Age}

\section{A. Assent Obtained:}

The assent discussion was initiated on

(date) at (time).

The information was presented in age-appropriate terms

Minor Subject's Printed Name

Minor Subject's Signature (8-13 years)

\section{$\overline{\text { Minor Subject's Date of Birth }}$}

$\overline{\text { Date }} \overline{\text { Time }}$

* Please note that the parent(s)/legally authorized representative(s) must sign the consent signature page.

I hereby certify that I have discussed the research project with the minor subject and/or his/her parent(s)/legally authorized representative(s). I have explained all the information contained in the informed consent document, including any risks that may be reasonably expected to occur. I further certify that the research subject was encouraged to ask questions and that all questions were answered.

Printed Name of Person Obtaining Assent

Signature of Person Obtaining Assent

$$
\overline{\text { Date }} \overline{\text { Time }}
$$

B. Assent Not Obtained, but Minor Subject was Enrolled:

Assent of the minor subject was NOT obtained for the following reason:

$\square$ Minor is cognitively or emotionally unable to participate in an assent discussion (e.g., minor has either a psychiatric or developmental disorder; minor received narcotics within the last 4 hours; minor is sedated; etc.).

$\square$ Minor refused to provide assent; however, the intervention or procedure involved in the research holds out a prospect of direct benefit that is important to the health or well being of the minor and is available only in the context of the research [45 CFR 46.408(a)].

C. Assent Was Obtained, but Minor Subject was Unable to Sign:

$\square$ The minor assented to participation, but has an incapacity that prevents applying a signature (e.g., the subject's dominant hand is incapacitated, the subject is illiterate, etc.) The assenting subject's inability to sign the assent document has been duly noted in the research record.

June 2, 2016

Page 8 of 8

Subject or Parent/Legally Authorized Representative Initials

IRB NUMBER: 16-04531-XP

IRB APPROVAL DATE: 06/06/2016

IRB EXPIRATION DATE: 04/14/2017 


\section{APPENDIX B. CERTIFICATE OF CONFIDENTIALITY}

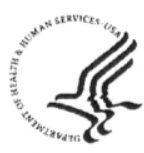

Public Health Service

Eunice Kennedy Shriver National

Institute of Child Health and

Human Development

6710B ROCKLEDGE DRIVE

$\mathrm{Rm} 2158$

Bethesda, MD 20817

$8 / 8 / 2016$

The University of Tennessee Health Science Center

Dr. Joyce Graff

910 Madison Avenue

Suite 823

Memphis, TN 38163

Dear Dr. Graff,

Enclosed is the Confidentiality Certificate, protecting the identity of research subjects in your singlesite/single-protocol project entitled "Health-Related Quality of Life in Female Children with Congenital Adrenal Hyperplasia (CAH): A Mixed Methods Study".

Please note that the Certificate expires on 04/14/2017.

Please be sure that the consent form given to research participants accurately states the intended uses of personally identifiable information and the confidentiality protections, including the protection provided by the Certificate of Confidentiality with its limits and exceptions.

If you determine that the research project will not be completed by the expiration date, 04/14/2017, you must submit a written request for an extension of the Certificate three (3) months prior to the expiration date. If you make any changes to the protocol for this study, you should contact me regarding modification of this Certificate. Any requests for modifications of this Certificate must include the reason for the request, documentation of the most recent IRB approval, and the expected date for completion of the research project.

Please advise me of any situation in which the certificate is employed to resist disclosure of information in legal proceedings. Should attorneys for the project wish to discuss the use of the certificate, they may contact the Office of the NIH Legal Advisor, National Institutes of Health, at (301) 496-6043.

Correspondence should be sent to:

Approved Date: 08/08/2016
Dennis Twombly Ph.D.

Eunice Kennedy Shriver National Institute of Child

Health and Human Development

6710B ROCKLEDGE DRIVE

$\mathrm{Rm} 2158$

Bethesda, MD 20817

Sincerely,

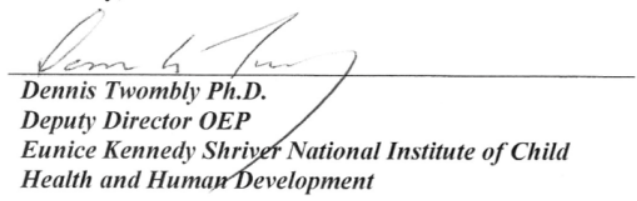


hyperplasia. Twenty five children and adolescents and their caregiver will be invited to participate in semi-structured interviews.

A Certificate of Confidentiality is needed because sensitive information will be collected during the course of the study. The certificate will help researchers avoid involuntary disclosure that could expose subjects or their families to adverse economic, legal, psychological and social consequences.

Participants are coded by numbers not by names. Linking information will be secured in a locked file cabinet. Questionnaires and audio recordings will be stored in a locked file cabinet located in the investigator's office. Electronic data will be password protected. Identifiers will be destroyed when the study is completed. Participant names will not appear in presentations or publications that arise from the project. Access to data will be limited to study personnel who have been approved by the university Institutional Review Board.

This research begins on 07/08/2016, and is expected to end on 04/14/2017.

As provided in section 301 (d) of the Public Health Service Act 42 U.S.C. 241(d):

"Persons so authorized to protect the privacy of such individuals may not be compelled in any Federal, State, or local civil, criminal, administrative, legislative, or other proceedings to identify such individuals."

This Certificate does not protect you from being compelled to make disclosures that: (1) have been consented to in writing by the research subject or the subject's legally authorized representative; (2) are required by the Federal Food, Drug, and Cosmetic Act (21 U.S.C. 301 et seq.) or regulations issued under that Act; or (3) have been requested from a research project funded by NIH or DHHS by authorized representatives of those agencies for the purpose of audit or program review.

This Certificate does not represent an endorsement of the research project by the Department of Health and Human Services. This Certificate is now in effect and will expire on 04/14/2017. The protection afforded by this Confidentiality Certificate is permanent with respect to any individual who participates as a research subject (i.e., about whom the investigator maintains identifying information) during the time the Certificate is in effect.

Signed Date:
Sincerely,

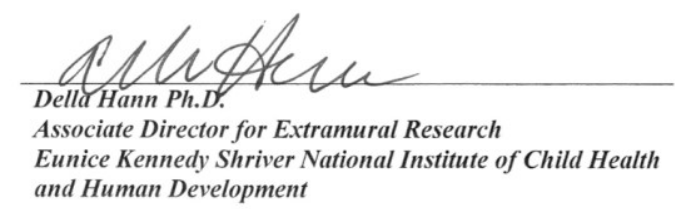


hyperplasia. Twenty five children and adolescents and their caregiver will be invited to participate in semi-structured interviews.

A Certificate of Confidentiality is needed because sensitive information will be collected during the course of the study. The certificate will help researchers avoid involuntary disclosure that could expose subjects or their families to adverse economic, legal, psychological and social consequences.

Participants are coded by numbers not by names. Linking information will be secured in a locked file cabinet. Questionnaires and audio recordings will be stored in a locked file cabinet located in the investigator's office. Electronic data will be password protected. Identifiers will be destroyed when the study is completed. Participant names will not appear in presentations or publications that arise from the project. Access to data will be limited to study personnel who have been approved by the university Institutional Review Board.

This research begins on 07/08/2016, and is expected to end on 04/14/2017.

As provided in section 301 (d) of the Public Health Service Act 42 U.S.C. 241(d):

"Persons so authorized to protect the privacy of such individuals may not be compelled in any Federal, State, or local civil, criminal, administrative, legislative, or other proceedings to identify such individuals."

This Certificate does not protect you from being compelled to make disclosures that: (1) have been consented to in writing by the research subject or the subject's legally authorized representative; (2) are required by the Federal Food, Drug, and Cosmetic Act (21 U.S.C. 301 et seq.) or regulations issued under that Act; or (3) have been requested from a research project funded by NIH or DHHS by authorized representatives of those agencies for the purpose of audit or program review.

This Certificate does not represent an endorsement of the research project by the Department of Health and Human Services. This Certificate is now in effect and will expire on 04/14/2017. The protection afforded by this Confidentiality Certificate is permanent with respect to any individual who participates as a research subject (i.e., about whom the investigator maintains identifying information) during the time the Certificate is in effect.

Signed Date:
Sincerely,

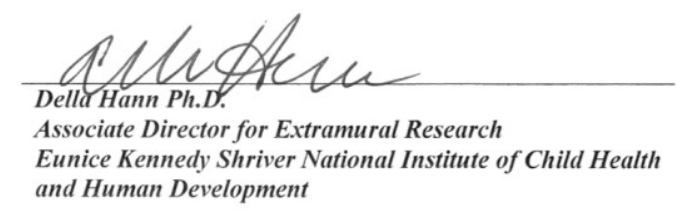




\section{Volunteers Needed}

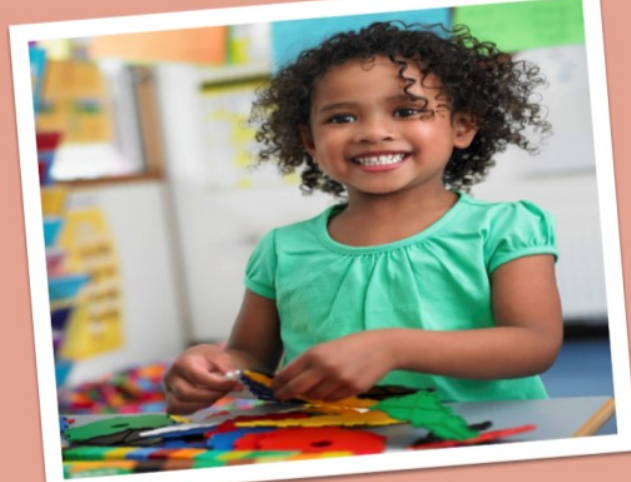

You are invited to participate in a research study that aims to

learn more about the health of female children and adolescents with congenital adrenal hyperplasia (CAH) and how CAH affects the child's life.

For more information contact: Lacretia Carroll PhD (c), RN

Phone: 901-232-1682

Email: lcarrol6@uthsc.edu Surveys and intervieus may be completed over the phone or in person at private, easily accessed locations

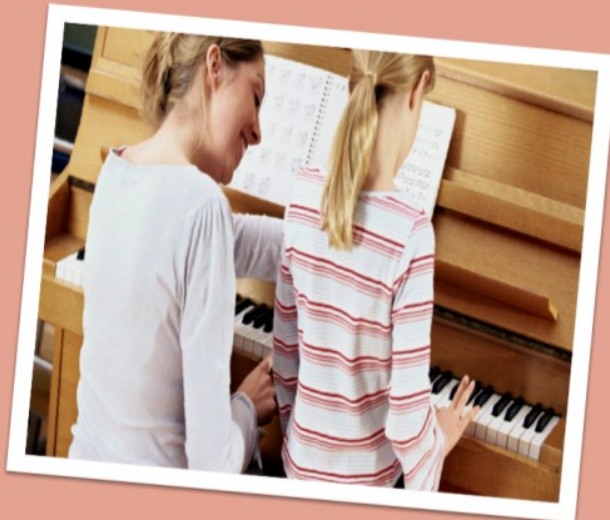

You may participate if you:

Are a female between the ages of 7-18 years old diagnosed with $\mathrm{CAH}$

Understand and speak English

Are a parent or caregiver over the age of 18 who cares for a female child or adolescent with CAH

\section{Participation may include:}

Completion of a survey

A 2-hour interview

Participants will receive a $\$ 20$ gift card for completing the interview and survey.

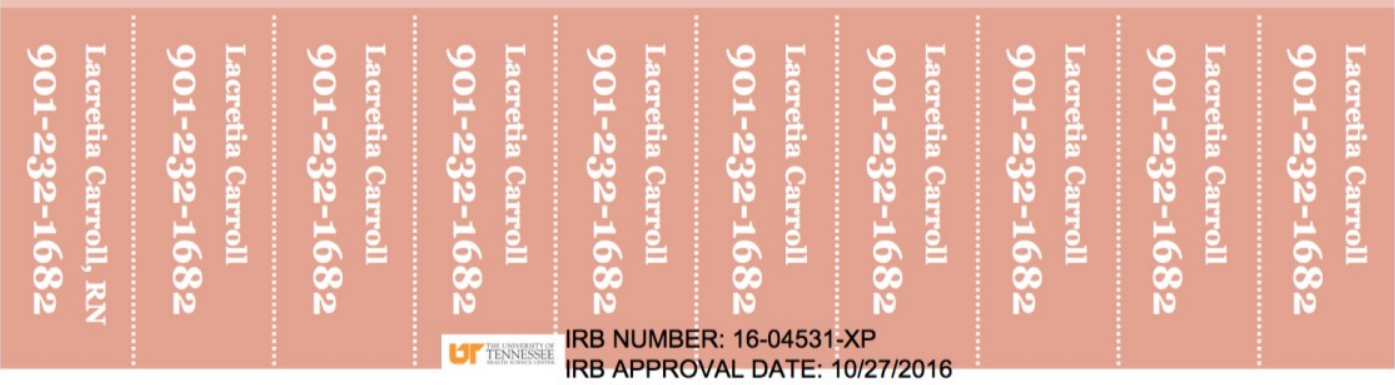




\section{APPENDIX D. PARTICIPANT INTEREST LETTER}

April 6, 2016

$\mathrm{Hi}$,

My name is Lacretia Carroll. I am a nurse who wants to learn more about congenital adrenal hyperplasia. I am doing a research study about the health of female children and adolescents with congenital adrenal hyperplasia, and how congenital adrenal hyperplasia affects their life. For this study I will meet with children and adolescents and one caregiver who knows the child well. The caregiver may be the child's mother or father but does not have to be. The person should know the child well enough to answer questions about the child's health, friends, activities and overall well-being. The child with congenital adrenal hyperplasia must be between the 7 years old and 18 years old. Both the child/adolescent and caregiver will answer questions. Our meeting will last 2 hours.

Each person who meets with me will receive a $\$ 20$ gift card for your time and as a thank you for participating in the study.

Please email me at lcarrol6@uthsc.edu or call me at 901-232-1682 to find out more about this study.

Thank you for your time!

Lacretia Carroll RN, MSN

$\mathrm{PhD}$ in Nursing Science Student

University of Tennessee Health Science Center

901-232-1682 


\section{APPENDIX E. DEMOGRAPHIC FORM}

\section{Demographic Form}

Please answer the following questions about yourself (parent/caregiver)

1. What is your age?

2. What is your gender? (Please select one)

Female

Male

3. What is your ethnicity (Please select one)

American Indian or Alaska Native

Asian

Black or African American

Hispanic or Latino

Native Hawaiian or Pacific Islander

White

Other (please specify)

4. What is your marital status? (Please select one)

Single

Married

Divorced

Widowed

5. What is your relationship to the child with congenital adrenal hyperplasia? (Please select one)

Parent

Grandparent

UT IRB NUMBER: 16-04531-XP

IRB APPROVAL DATE: 06/06/2016 
Sibling

Other family relationship (please specify)

6. What is the highest level of education you have completed? (Please select one)

Some high school

High school diploma or GED

Some college

Associate's degree

Bachelor's degree

Graduate/Master's Degree

Doctoral Degree

7. What is your employment status? (Please select one)

Full- time (30 hours or more per week)

Part- time (29 hours or less per week)

Stay at home caregiver

Unemployed

Retired

8. What city and state do you live in?

City State

9. On average, how often do you talk with the child with congenital adrenal hyperplasia about her diagnosis? (Check all that apply)

Daily

Once or twice a week

Three to four times a week

IRB NUMBER: 16-04531-XP
IRB APPROVAL DATE: 06/06/2016 
Monthly

Twice a year

Three for four times a year

Please answer the following questions about the child with congenital adrenal hyperplasia:

1. What is her age?

2. What is her diagnosis? (Please select one)

Classical CAH

Non-classical CAH

3. What is her ethnicity (Please select one)

American Indian or Alaska Native

Asian

Black or African American

Hispanic or Latino

Native Hawaiian or Pacific Islander

White

Other (please specify)

4. What grade is the child with congenital adrenal hyperplasia currently enrolled in?

5. Does she receive special services at school? (Please select one)

Yes

No

If yes, please explain.

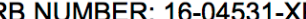

IRB APPROVAL DATE: 06/06/2016 
6. Is the child with congenital adrenal hyperplasia involved in extracurricular activities? (Please select one)

Yes $\quad$ No

If yes, what are the extracurricular activities she is involved in? (Please list)

7. What types of doctors has the child visited in the last year?

Pediatrician

Endocrinologist

Urologist

Geneticist

Psychologist

Other (please specify)

8. How often does the child with congenital adrenal hyperplasia take hydrocortisone?

Once a day

Twice a day

Three times a day

Other

9. On average, how often does the child with congenital adrenal hyperplasia receive stress doses of steroids?

Once a week

Twice a week

More than twice a week

Monthly

IRB APPROVAL DATE: 06/06/2016 
Twice a month

Three times a month

Once a year

Twice a year

Other

10. Does the child with congenital adrenal hyperplasia live with you?

Yes

No

If no, on average how many hours do you spend each week with her?

11. On average, how often does the child with congenital adrenal hyperplasia visit the Emergency Department?

Once a month

Twice a month

Once a year

Twice a year

Other

12. Does the child with congenital adrenal hyperplasia have other health issues? (Please select one)

Yes No

If yes, please explain.

13. What type of health insurance does the child have?

Private

Medicaid

Other

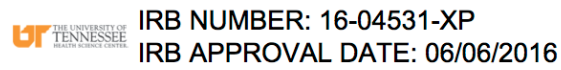


None

14. On a scale from 0 to 10 , please rate the health of the child with congenital adrenal hyperplasia. (Circle your answer)

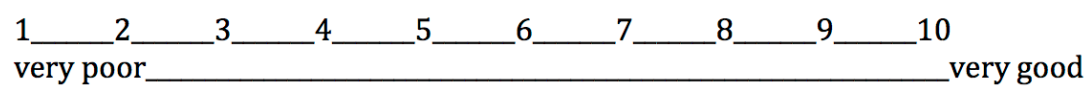




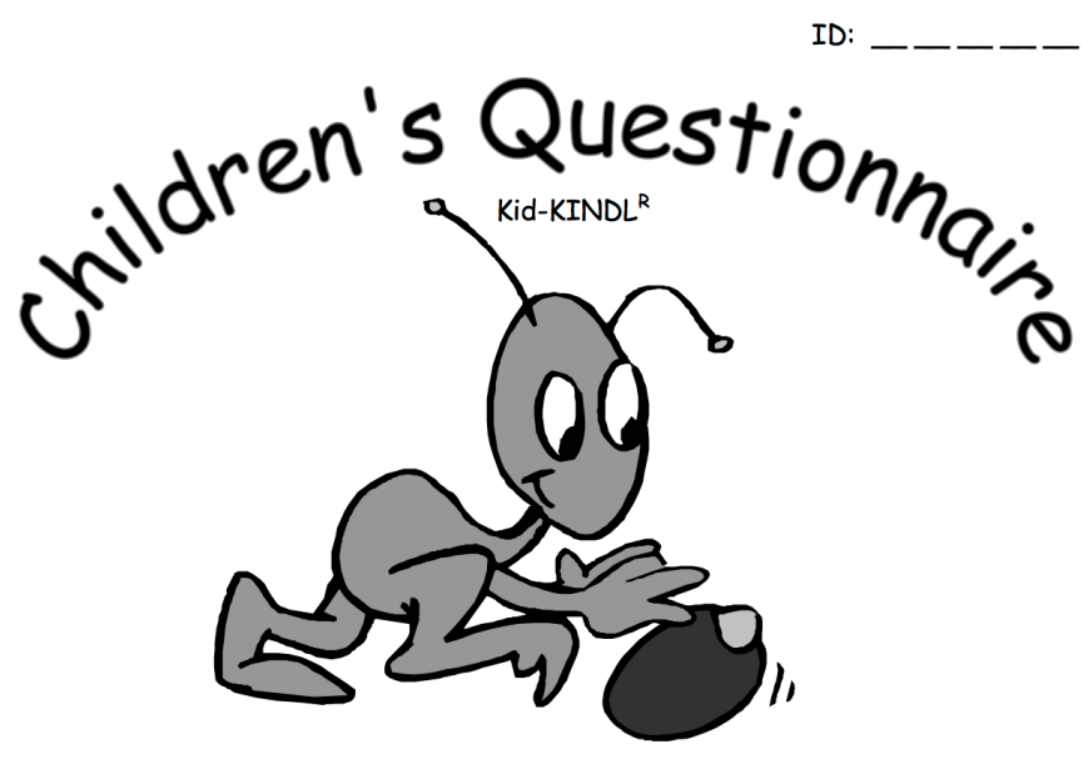

Hello there!

we would like to know how you have been feeling during the past week, so we have worked out a few questions which we would like you to answer.

$\Rightarrow$ Please read each question carefully.

$\Rightarrow$ Think about how things have been for you over the past week.

$\Rightarrow$ Choose the answer that fits you best in each line and put a cross in the box.

There are no right or wrong answers. It's what you think that matters.

\begin{tabular}{|l|c|c|c|c|c|}
\hline For example: & never & seldom & $\begin{array}{c}\text { some- } \\
\text { times }\end{array}$ & often & $\begin{array}{c}\text { all the } \\
\text { time }\end{array}$ \\
\hline $\begin{array}{l}\text { During the past week, I liked to } \\
\text { listen to music. }\end{array}$ & $\square$ & $\square$ & $\square$ & $\bigotimes$ & $\square$ \\
\hline
\end{tabular}

Date of fill out:

(day / month / year)

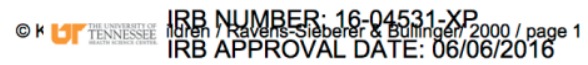


Please tell us something about you. Please put a cross or fill in!

I am a

$\square$ girl $\square$ boy

Age: years old

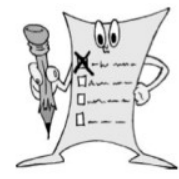

How many siblings do you have? $\quad \square 0 \quad \square 1 \quad \square 2 \square 3 \quad \square 4 \quad \square 5 \square$ more than 5

Which type of school do you go to?

1. First of all, we would like to know something about your physical health...

\begin{tabular}{|c|c|c|c|c|c|c|}
\hline & During the past week... & never & seldom & $\begin{array}{l}\text { some- } \\
\text { times }\end{array}$ & often & $\begin{array}{l}\text { all the } \\
\text { time }\end{array}$ \\
\hline 1. & ... I felt ill & $\square$ & $\square$ & $\square$ & $\square$ & $\square$ \\
\hline 2. & ... I had a headache or tummy-ache & $\square$ & $\square$ & $\square$ & $\square$ & $\square$ \\
\hline 3. & ... I was tired and worn-out & $\square$ & $\square$ & $\square$ & $\square$ & $\square$ \\
\hline 4. & ... I felt strong and full of energy & $\square$ & $\square$ & $\square$ & $\square$ & $\square$ \\
\hline
\end{tabular}

2. ... then something about how you've been feeling in general...

\begin{tabular}{|l|c|c|c|c|c|}
\hline$\quad$ During the past week... & never & seldom & $\begin{array}{c}\text { some- } \\
\text { times }\end{array}$ & often & $\begin{array}{c}\text { all the } \\
\text { time }\end{array}$ \\
\hline 1. $\quad \ldots$ I had fun and laughed a lot & $\square$ & $\square$ & $\square$ & $\square$ & $\square$ \\
\hline 2. $\quad \ldots$ I was bored & $\square$ & $\square$ & $\square$ & $\square$ & $\square$ \\
\hline 3. $\quad \ldots$ I felt alone & $\square$ & $\square$ & $\square$ & $\square$ & $\square$ \\
\hline $4 . \quad \ldots$ I was scared & $\square$ & $\square$ & $\square$ & $\square$ & $\square$ \\
\hline
\end{tabular}

3. ... and how you have been feeling about yourself.

\begin{tabular}{|c|c|c|c|c|c|c|}
\hline & During the past week... & never & seldom & $\begin{array}{c}\text { some- } \\
\text { times }\end{array}$ & often & $\begin{array}{c}\text { all the } \\
\text { time }\end{array}$ \\
\hline 1. & ... I was proud of myself & $\square$ & $\square$ & $\square$ & $\square$ & $\square$ \\
\hline 2. & $\ldots$ I felt on top of the world & $\square$ & $\square$ & $\square$ & $\square$ & $\square$ \\
\hline 3. & ... I felt pleased with myself & $\square$ & $\square$ & $\square$ & $\square$ & $\square$ \\
\hline 4. & ... I had lots of good ideas & $\square$ & $\square$ & $\square$ & $\square$ & $\square$ \\
\hline
\end{tabular}

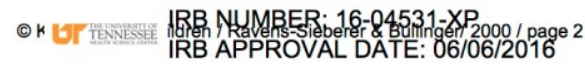


4. The next questions are about your family ...

\begin{tabular}{|l|c|c|c|c|c|}
\hline$\quad$ During the past week... & never & seldom & $\begin{array}{c}\text { some- } \\
\text { times }\end{array}$ & often & $\begin{array}{c}\text { all the } \\
\text { time }\end{array}$ \\
\hline 1. $\quad$... I got on well with my parents & $\square$ & $\square$ & $\square$ & $\square$ & $\square$ \\
\hline 2. $\quad$... I felt fine at home & $\square$ & $\square$ & $\square$ & $\square$ & $\square$ \\
\hline 3. ... We quarrelled at home & $\square$ & $\square$ & $\square$ & $\square$ & $\square$ \\
\hline $4 . \quad \begin{array}{l}\text {.. My parents stopped me from } \\
\text { doing certain things }\end{array}$ & $\square$ & $\square$ & $\square$ & $\square$ & $\square$ \\
\hline
\end{tabular}

5. ... and then about friends.

\begin{tabular}{|c|c|c|c|c|c|c|}
\hline & During the past week... & never & seldom & $\begin{array}{l}\text { some- } \\
\text { times }\end{array}$ & often & $\begin{array}{l}\text { all the } \\
\text { time }\end{array}$ \\
\hline 1. & ... I played with friends & $\square$ & $\square$ & $\square$ & $\square$ & $\square$ \\
\hline 2. & ... Other kids liked me & $\square$ & $\square$ & $\square$ & $\square$ & $\square$ \\
\hline 3. & ... I got along well with my friends & $\square$ & $\square$ & $\square$ & $\square$ & $\square$ \\
\hline 4. & ... I felt different from other children & $\square$ & $\square$ & $\square$ & $\square$ & $\square$ \\
\hline
\end{tabular}

6. Last of all, we would like to know something about school.

\begin{tabular}{|l|c|c|c|c|c|}
\hline $\begin{array}{l}\text { During the last week in which I was } \\
\text { at school... }\end{array}$ & never & seldom & $\begin{array}{c}\text { some- } \\
\text { times }\end{array}$ & often & $\begin{array}{c}\text { all the } \\
\text { time }\end{array}$ \\
\hline 1. $\quad$.. doing my schoolwork was easy & $\square$ & $\square$ & $\square$ & $\square$ & $\square$ \\
\hline 2. $\quad \ldots$ I enjoyed my lessons & $\square$ & $\square$ & $\square$ & $\square$ & $\square$ \\
\hline 3. $\quad \ldots$ I worried about my future & $\square$ & $\square$ & $\square$ & $\square$ & $\square$ \\
\hline $4 . \quad \begin{array}{l}\text {... I worried about bad marks or } \\
\text { grades }\end{array}$ & $\square$ & $\square$ & $\square$ & $\square$ & $\square$ \\
\hline
\end{tabular}

Thank you for helping us!

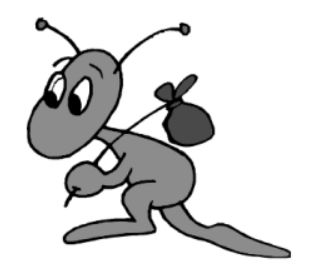

IRB NWMBER 16-04531-XP
○ k UT TENESEE
IRB APPROVAL DATE: $06 / 06 / 2016$ 
ID:

\section{Questionnaire for adolescents}

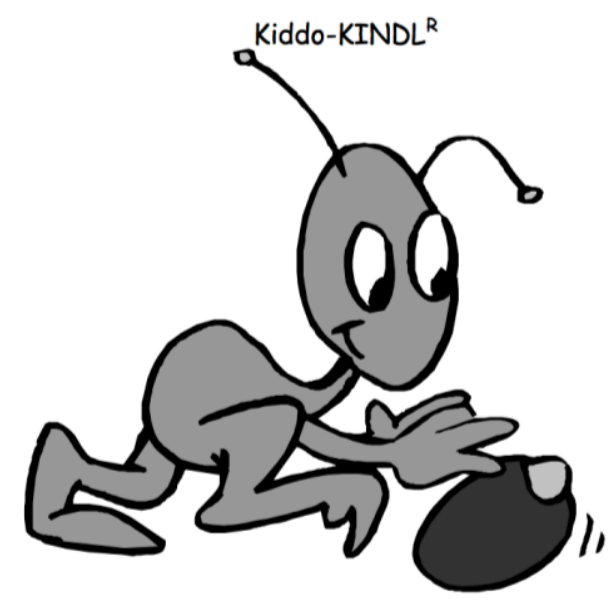

Hello there!

we would like to know how you have been feeling during the past week, so we have worked out a few questions which we would like you to answer.

$\Rightarrow$ Please read each question carefully.

$\Rightarrow$ Think about how things have been for you over the past week.

$\Rightarrow$ Choose the answer that fits you best in each line and put a cross in the box.

There are no right or wrong answers. It's what you think that matters.

\begin{tabular}{|l|c|c|c|c|c|}
\hline For example: & never & seldom & $\begin{array}{c}\text { some- } \\
\text { times }\end{array}$ & often & $\begin{array}{c}\text { all the } \\
\text { time }\end{array}$ \\
\hline $\begin{array}{l}\text { During the past week, I liked to } \\
\text { listen to music }\end{array}$ & $\square$ & $\square$ & $\square$ & $\bigotimes$ & $\square$ \\
\hline
\end{tabular}

Date of fill out:

(day / month / year) 
Please tell us something about you. Please put a cross or fill in!

I am a

$$
\square \text { girl } \square \text { boy }
$$

Age: years old

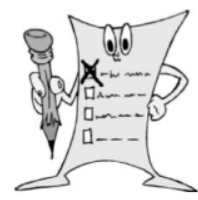

How many siblings do you have? $\quad \square 0 \square 1 \square 2 \square 3 \square 4 \square 5 \square$ more than 5

Which type of school do you go to?

1. First of all, we would like to know something about your physical health...

\begin{tabular}{|c|c|c|c|c|c|c|}
\hline & During the past week... & never & seldom & $\begin{array}{c}\text { some- } \\
\text { times }\end{array}$ & often & $\begin{array}{l}\text { all the } \\
\text { time }\end{array}$ \\
\hline 1. & ... I felt ill & $\square$ & $\square$ & $\square$ & $\square$ & $\square$ \\
\hline 2. & ... I was in pain & $\square$ & $\square$ & $\square$ & $\square$ & $\square$ \\
\hline 3. & ... I was tired and worn-out & $\square$ & $\square$ & $\square$ & $\square$ & $\square$ \\
\hline 4. & ... I felt strong and full of energy & $\square$ & $\square$ & $\square$ & $\square$ & $\square$ \\
\hline
\end{tabular}

\section{2. ... then something about how you've been feeling in general...}

\begin{tabular}{|l|c|c|c|c|c|}
\hline$\quad$ During the past week... & never & seldom & $\begin{array}{c}\text { some- } \\
\text { times }\end{array}$ & often & $\begin{array}{c}\text { all the } \\
\text { time }\end{array}$ \\
\hline 1. $\quad$... I had fun and laughed a lot & $\square$ & $\square$ & $\square$ & $\square$ & $\square$ \\
\hline 2. $\quad \ldots$ I was bored & $\square$ & $\square$ & $\square$ & $\square$ & $\square$ \\
\hline 3. $\quad \ldots$ I felt alone & $\square$ & $\square$ & $\square$ & $\square$ & $\square$ \\
\hline 4. $\quad$... I felt scared or unsure of myself & $\square$ & $\square$ & $\square$ & $\square$ & $\square$ \\
\hline
\end{tabular}

3. ... and how you have been feeling about yourself.

\begin{tabular}{|c|c|c|c|c|c|c|}
\hline & During the past week... & never & seldom & $\begin{array}{l}\text { some- } \\
\text { times }\end{array}$ & often & $\begin{array}{l}\text { all the } \\
\text { time }\end{array}$ \\
\hline 1. & ... I was proud of myself & $\square$ & $\square$ & $\square$ & $\square$ & $\square$ \\
\hline 2. & $\ldots$ I felt on top of the world & $\square$ & $\square$ & $\square$ & $\square$ & $\square$ \\
\hline 3. & ... I felt pleased with myself & $\square$ & $\square$ & $\square$ & $\square$ & $\square$ \\
\hline 4. & ... I had lots of good ideas & $\square$ & $\square$ & $\square$ & $\square$ & $\square$ \\
\hline
\end{tabular}


4. The next questions are about your family ...

\begin{tabular}{|c|c|c|c|c|c|c|}
\hline & During the past week... & never & seldom & $\begin{array}{l}\text { some- } \\
\text { times }\end{array}$ & often & $\begin{array}{c}\text { all the } \\
\text { time }\end{array}$ \\
\hline 1. & ... I got on well with my parents & $\square$ & $\square$ & $\square$ & $\square$ & $\square$ \\
\hline 2. & $\ldots$ I felt fine at home & $\square$ & $\square$ & $\square$ & $\square$ & $\square$ \\
\hline 3. & ... We quarrelled at home & $\square$ & $\square$ & $\square$ & $\square$ & $\square$ \\
\hline 4. & ... I felt restricted by my parents & $\square$ & $\square$ & $\square$ & $\square$ & $\square$ \\
\hline
\end{tabular}

\section{5. ... and then about friends.}

\begin{tabular}{|l|c|c|c|c|c|}
\hline$\quad \begin{array}{l}\text { During the past week... } \\
\text { 1. } \quad \text {... I did things together with my } \\
\text { friends }\end{array}$ & $\square$ & $\square$ & $\square$ & $\square$ & $\square$ \\
\hline 2. $\quad \ldots$ I was a "success" with my friends & $\square$ & $\square$ & $\square$ & $\square$ & $\square$ \\
\hline $3 . \quad \ldots$ I got along well with my friends & $\square$ & $\square$ & $\square$ & $\square$ & $\square$ \\
\hline $4 . \quad$... I felt different from other people & $\square$ & $\square$ & $\square$ & $\square$ & $\square$ \\
\hline
\end{tabular}

6. Last of all, we would like to know something about school.

\begin{tabular}{|l|c|c|c|c|c|}
\hline $\begin{array}{l}\text { During the last week in which I was } \\
\text { at school... }\end{array}$ & never & seldom & $\begin{array}{c}\text { some- } \\
\text { times }\end{array}$ & often & $\begin{array}{c}\text { all the } \\
\text { time }\end{array}$ \\
\hline 1. $\quad$... doing the schoolwork was easy & $\square$ & $\square$ & $\square$ & $\square$ & $\square$ \\
\hline 2. $\quad$.. I found school interesting & $\square$ & $\square$ & $\square$ & $\square$ & $\square$ \\
\hline $3 . \quad \ldots$ I worried about my future & $\square$ & $\square$ & $\square$ & $\square$ & $\square$ \\
\hline $\begin{array}{l}\text { 4. } \quad \text {.. I worried about getting bad marks } \\
\text { or grades }\end{array}$ & $\square$ & $\square$ & $\square$ & $\square$ & $\square$ \\
\hline
\end{tabular}

Thank you for helping us!

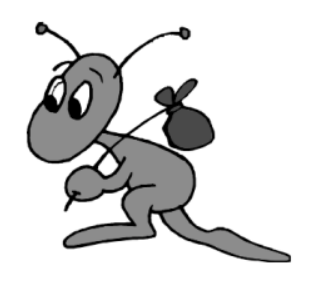




\section{APPENDIX H. PARENT KINDL-R QUESTIONNAIRE}

ID:

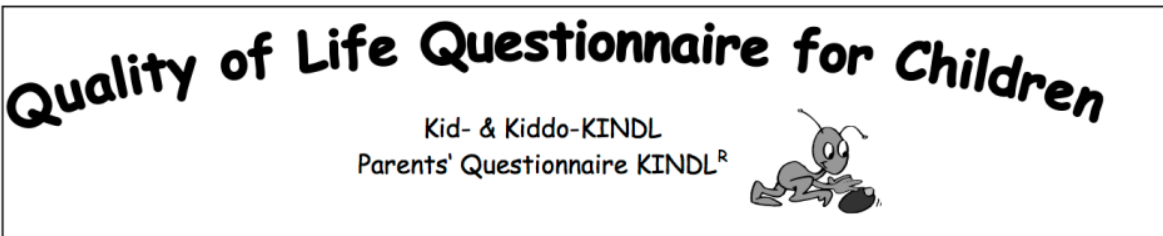

Dear Parent,

We really appreciate your taking the time to complete this questionnaire about your child's well-being and health-related quality of life.

Since it is a matter of your own assessment of your child's well-being, please complete the questionnaire yourself according to the instructions, i.e. without asking your child.
$\Rightarrow \quad$ Read each question carefully.
$\Rightarrow \quad$ Think about how your child has been feeling during the past week.
$\Rightarrow \quad$ Put a cross in the box corresponding to the answer in each line that fits your child best.

For example:

\begin{tabular}{|ccccccc}
\hline During the past week ... & never & seldom & $\begin{array}{c}\text { some- } \\
\text { times }\end{array}$ & often & all the \\
time
\end{tabular}

$\begin{array}{ll}\text { My Child is a: } & \square \text { Girl } \quad \square \text { Boy } \\ \text { Age: } & \quad \square \text { Mother } \quad \square \text { Father } \square \text { Other } \\ \text { You are: } & \text { Date of fill out: }\end{array}$




\section{Physical Well-being}

\begin{tabular}{|c|c|c|c|c|c|c|}
\hline & During the past week ... & never & seldom & $\begin{array}{l}\text { some- } \\
\text { times }\end{array}$ & often & $\begin{array}{l}\text { all the } \\
\text { time }\end{array}$ \\
\hline 1. & ... my child felt ill & $\square$ & $\square$ & $\square$ & $\square$ & $\square$ \\
\hline 2. & $\begin{array}{l}\text {... my child had a headache or tummy- } \\
\text { ache }\end{array}$ & $\square$ & $\square$ & $\square$ & $\square$ & $\square$ \\
\hline 3. & ... my child was tired and worn-out & $\square$ & $\square$ & $\square$ & $\square$ & $\square$ \\
\hline & $\begin{array}{l}\text {... my child felt strong and full of } \\
\text { energy }\end{array}$ & $\square$ & $\square$ & $\square$ & $\square$ & $\square$ \\
\hline
\end{tabular}

\section{Emotional Well-being}

\begin{tabular}{|ll|c|c|c|c|c|}
\hline$\quad$ During the past week ... & never & seldom & $\begin{array}{c}\text { some- } \\
\text { times }\end{array}$ & often & $\begin{array}{c}\text { all the } \\
\text { time }\end{array}$ \\
\hline 1. $\quad$... my child had fun and laughed a lot & $\square$ & $\square$ & $\square$ & $\square$ & $\square$ \\
\hline 2. $\quad \begin{array}{l}\text {... my child didn't feel much like doing } \\
\text { anything }\end{array}$ & $\square$ & $\square$ & $\square$ & $\square$ & $\square$ \\
\hline 3. $\quad \ldots$ my child felt alone & $\square$ & $\square$ & $\square$ & $\square$ & $\square$ \\
\hline $4 . \quad \begin{array}{l}\text {... my child felt scared or unsure of } \\
\text { him-/ herself }\end{array}$ & $\square$ & $\square$ & $\square$ & $\square$ & $\square$ \\
\hline
\end{tabular}

\section{Self-esteem}

\begin{tabular}{|c|c|c|c|c|c|c|}
\hline & During the past week ... & never & seldom & $\begin{array}{l}\text { some- } \\
\text { times }\end{array}$ & often & $\begin{array}{l}\text { all the } \\
\text { time }\end{array}$ \\
\hline 1. & ... my child was proud of him-/herself & $\square$ & $\square$ & $\square$ & $\square$ & $\square$ \\
\hline 2. & ... my child felt on top of the world & $\square$ & $\square$ & $\square$ & $\square$ & $\square$ \\
\hline 3. & $\begin{array}{l}\text {... my child felt pleased with him- } / \\
\text { herself }\end{array}$ & $\square$ & $\square$ & $\square$ & $\square$ & $\square$ \\
\hline 4. & ... my child had lots of good ideas & $\square$ & $\square$ & $\square$ & $\square$ & $\square$ \\
\hline
\end{tabular}

\section{Family}

\begin{tabular}{|c|c|c|c|c|c|c|}
\hline & During the past week ... & never & seldom & $\begin{array}{l}\text { some- } \\
\text { times }\end{array}$ & often & $\begin{array}{l}\text { all the } \\
\text { time }\end{array}$ \\
\hline 1. & $\begin{array}{l}\ldots \text { my child got on well with us as } \\
\text { parents }\end{array}$ & $\square$ & $\square$ & $\square$ & $\square$ & $\square$ \\
\hline 2. & ... my child felt fine at home & $\square$ & $\square$ & $\square$ & $\square$ & $\square$ \\
\hline 3. & ... we quarrelled at home & $\square$ & $\square$ & $\square$ & $\square$ & $\square$ \\
\hline & $\begin{array}{l}\ldots \text { my child felt that I was bossing him/ } \\
\text { her around }\end{array}$ & $\square$ & $\square$ & $\square$ & $\square$ & $\square$ \\
\hline
\end{tabular}




\section{Social Contacts}

\begin{tabular}{|c|c|c|c|c|c|c|}
\hline & During the past week ... & never & seldom & $\begin{array}{l}\text { some- } \\
\text { times }\end{array}$ & often & $\begin{array}{l}\text { all the } \\
\text { time }\end{array}$ \\
\hline 1. & $\begin{array}{l}\ldots \text { my child did things together with } \\
\text { friends }\end{array}$ & $\square$ & $\square$ & $\square$ & $\square$ & $\square$ \\
\hline 2. & ... my child was liked by other kids & $\square$ & $\square$ & $\square$ & $\square$ & $\square$ \\
\hline 3. & $\begin{array}{l}\text {... my child got along well with his/ her } \\
\text { friends }\end{array}$ & $\square$ & $\square$ & $\square$ & $\square$ & $\square$ \\
\hline 4. & $\begin{array}{l}\text {... my child felt different from other } \\
\text { children }\end{array}$ & $\square$ & $\square$ & $\square$ & $\square$ & $\square$ \\
\hline
\end{tabular}

6. School

\begin{tabular}{|l|c|c|c|c|c|c|}
\hline & $\begin{array}{c}\text { During the last week in which my child } \\
\text { was at school ... }\end{array}$ & never & seldom & $\begin{array}{c}\text { some- } \\
\text { times }\end{array}$ & often & $\begin{array}{c}\text { all the } \\
\text { time }\end{array}$ \\
\hline 1. $\quad \begin{array}{c}\text {... my child easily coped with } \\
\text { schoolwork }\end{array}$ & $\square$ & $\square$ & $\square$ & $\square$ & $\square$ \\
\hline 2. $\quad$... my child enjoyed the school lessons & $\square$ & $\square$ & $\square$ & $\square$ & $\square$ \\
\hline 3. $\quad$... my child worried about his/her future & $\square$ & $\square$ & $\square$ & $\square$ & $\square$ \\
\hline 4. $\quad$... my child was afraid of bad marks or \\
grades
\end{tabular}

Thank you for your co-operation! 


\section{APPENDIX I. INTERVIEW GUIDE FOR CHILD/ADOLESCENT}

\section{Interview Guide for Child/Adolescent}

Thank you for agreeing to be interviewed today. The purpose of this interview is to understand what it is like to live with congenital adrenal hyperplasia (CAH). I am interested in learning more about your health. I also want to learn what you think affects your health and how you feel about your health.

The interview will last no more than two hours. The information that you share with me today will be kept confidential. That means that the answers you give will only be shared with the researchers working with me. You answers will not be shared in a way that will tell people who you are. The information you share with me today will be used to better understand the health of kids with congenital adrenal hyperplasia. Your answers to questions and what you tell me will be used along with information from other kids with congenital adrenal hyperplasia, as a group. So, your answers will not be able to be traced back to you. You can stop the interview at any time, take a break, or choose to not answer any question(s) that make you feel uncomfortable.

I want to be sure that I get all the information you are sharing with me, so I would like to record my conversation with you today. However, if you do not want the conversation to be recorded you may still take part in the interview. When we finish talking, I will summarize what I heard you say and ask you if this is correct. I will also ask you if there is anything else you would like to share. What you say on the recording will be written out and your name will be removed. So no one will be able to connect your name to what you said to me. After the recording is written out, the recording will be destroyed.

Are you okay with me recording this interview?

1. I will begin by asking you to think about how you and (name of caregiver) talk about congenital adrenal hyperplasia.

a. How did you first talk with (name of caregiver) about congenital adrenal hyperplasia?

b. How comfortable are you talking with (name of caregiver) about congenital adrenal hyperplasia?

c. How much do you think you know about congenital adrenal hyperplasia? How have you learned about congenital adrenal hyperplasia?

d. How involved are you in the management of congenital adrena hyperplasia?

2. Tell me about others such as family members or other people in your life.

a. Do they know about your diagnosis? If yes, what do they know?

b. Do others talk with you about your diagnosis?

c. How did you first talk with others about your diagnosis? 
d. How comfortable are you talking with others about congenital adrenal hyperplasia?

e. How much information do you share with others about your diagnosis?

f. How do you decide how much you want to share with others?

g. Has knowing about congenital adrenal hyperplasia changed the way other people get along with you?

h. Do any of these people help you or (name of caregiver)? If yes, how?

3. Tell me about your friends.

a. Do they know about your diagnosis? If yes, what do they know? If no, why do you think they do not know about congenital adrenal hyperplasia?

b. Has knowing about congenital adrenal hyperplasia changed the way your friends get along with you? How do you feel about your friends knowing about your condition?

4. Tell me about your health?

a. How would you compare your health to the health of other kids who are your age or close to you in age?

b. Do you think congenital adrenal hyperplasia keeps you from doing anything? Please explain.

5. What is living with congenital adrenal hyperplasia like?

a. What is a good day like for you?

b. What is a bad day like for you?

c. What makes you feel good about yourself? About others?

d. What makes you feel bad about yourself? About others?

6. Some kids are easier to get along with than other kids. Some days are easier for kids than other days. Tell me about yourself. Do you think you are usually easy or difficult to get along with? Explain.

7. Overall, how do you feel about yourself?

a. Do you ever feel embarrassed?

b. Are there certain things you worry or fear more than others? Please explain.

c. Do you ever think about being different from other kids? Please explain.

8. Next I will ask you about the community in which you and (name of caregiver) live.

a. Tell me a story or a time when living with congenital adrenal hyperplasia has influenced your feelings about really belonging to the community.

IRB NUMBER: 16-04531-XP

IRB APPROVAL DATE: 06/06/2016 
b. Tell me a story or a time when living with congenital adrenal hyperplasia has influenced your feelings about not belonging to the community.

c. Has your diagnosis changed how you participate in school or community activities? Has your diagnosis changed how your family participates in school or community activities? Please explain.

9. What things are the most important in your life now?

a. What do you think can be done to help kids with congenital adrenal hyperplasia?

b. What should nurses and doctors and other health providers know about kids with congenital adrenal hyperplasia?

10. I am going to tell you some things I heard you say today. (The researcher will summarize what the participant shared during this interview in two or three sentences.)

a. Is what I just shared with you correct?

b. Is there anything that I missed or did not cover that is important? Please explain.

a. Is there anything that I just shared with you that is not really important for kids with congenital adrenal hyperplasia and their families? Please explain.

Thank you for taking the time to participate in this interview.

IRB NUMBER: 16-04531-XP
IRB APPROVAL DATE: 06/06/2016 


\title{
APPENDIX J. INTERVIEW GUIDE FOR CAREGIVER/PARENT
}

\author{
Interview Guide- Caregiver/Parent
}

Thank you for agreeing to be interviewed today. The purpose of this interview is to understand what it is like for a child to live with congenital adrenal hyperplasia (CAH). More specifically, I am interested in the health-related quality of life of children diagnosed with $\mathrm{CAH}$ from the perspective of the caregiver and the child. Health-related quality of life means a person's overall well-being and enjoyment or satisfaction with life. As such, I also hope to gain more insight about the factors that influence the child's health-related quality of life.

The interview will last no more than two hours. The information that you share with us is completely confidential. You will be assigned a code and the answers you provide will not have any identifying information. All information that is provided will only be shared with members of the study staff. The information I obtain today will be used to increase the awareness and knowledge about the health related quality of life of children with congenital adrenal hyperplasia. Should any information gathered during our interviews be used in scientific publications or presentations, it will be shared only as group information and individual participants will not in any way be identifiable. You can stop the interview at any time, take a break, or choose to not answer any question(s) that make you feel uncomfortable.

To ensure that I am able to capture accurate and complete responses, I would like to record this interview. However, if you do not want the conversation to be recorded you may still take part in the interview. At the end of the interview, I will summarize what I heard and ask you if this is correct. I will also ask you if there is any additional information you would like to share. As previously mentioned, all identifying information will be removed to protect your identity. The information that is recorded will be transcribed. After transcription, the recording will be destroyed.

Are you okay with me recording this interview?

1. I will begin by asking you to think about how you and (name of child) talk about congenital adrenal hyperplasia.

a. How did you first talk with (name of child) about congenital adrenal hyperplasia?

b. How comfortable are you talking with (name of child) about congenital adrenal hyperplasia?

c. How do you decide how much information to share with (name of child)?

d. How involved is (name of child) in the management of congenital adrenal hyperplasia?

2. Tell me about others such as family members, friends, or other people in your life.

a. Do they know about (name of child)'s diagnosis? If yes, what do they know?

IRB NUMBER: 16-04531-XP

IRB APPROVAL DATE: 06/06/2016 
b. How do they respond?

c. How did you first talk with others about (name of child)'s diagnosis?

d. How comfortable are you talking with others about congenital adrenal hyperplasia?

e. How much information do you share with others about (name of child)'s diagnosis?

f. How do you decide how much information to share with others?

g. Has knowing about (name of child)'s congenital adrenal hyperplasia changed the way they interact or get along with your child?

h. Are any of these people involved in helping you or your child?

3. Tell me about (name of child)'s friends.

a. Do they know about her diagnosis? If yes, how do they respond? If no, why do you think they do not know about congenital adrenal hyperplasia?

b. Has knowing about congenital adrenal hyperplasia changed the way they interact or get along with her? How do you think (name of child) feels about her friend's knowledge about her condition?

4. Tell me about (name of child)'s current health?

a. How would you compare her health to the health of other children her age?

b. Do you think congenital adrenal hyperplasia prevents her from doing anything? Please explain.

5. What you think living with congenital adrenal hyperplasia is like for (name of child)?

a. What is a good day like for (name of child)?

b. What is a bad day like for (name of child)?

c. What makes her feel good about herself? About others?

d. What makes her feel bad about herself? About others?

6. Some children are easier to get along with than other children. Some days are easier for children (and adults) than other days.

a. Tell me about (name of child).

b. Do you think she is usually easy or difficult to get along with? Explain.

7. Overall, how do you think (name of child) feels about herself?

a. Does she ever feel embarrassed?

b. Are there certain things she worries or fears more than others? Please explain.

c. Does she ever talk about being different from other girls?

8. Next I will ask you about the community in which you and (name of child) live.

a. Tell me a story or a time that you think living with congenital adrenal hyperplasia has influenced (name of child)'s feelings that she really belonged to the community.

IRB NUMBER: 16-04531-XP

IRB APPROVAL DATE: 06/06/2016 
b. Tell me a story or a time that you think living with congenital adrenal hyperplasia influenced (name of child)'s feeling that she did not belong to the community.

c. How has (name of child)'s diagnosis changed you and your family's participation in school or community activities? Please explain.

9. What are some things that you think are most important for (name of the child)?

a. What do you think can be done to improve the lives of children with congenital adrenal hyperplasia?

b. What should health providers know about children with congenital adrenal hyperplasia?

10. I will summarize what I heard you say today. (The researcher will summarize what the participant shared during this interview in two or three sentences).

a. Is this correct?

b. Are there any issues that I missed or did not cover that are important? Please explain.

c. Are there any issues that I covered that are not really important for children with congenital adrenal hyperplasia and their families? Please explain.

Thank you for taking the time to participate in this interview. 


\section{APPENDIX K. ONLINE SURVEY CONSENT}

Survey for Parents/Caregivers Health-Related Quality of Life of Female Children with Congenital Adrenal Hyperplasia: A Mixed Methods Study

You are being given the opportunity to participate in this research study. This survey is a part of a research study conducted by Dr. Carolyn Graff RN, PhD and Lacretia Carroll $\mathrm{RN}, \mathrm{PhD}(\mathrm{c})$. The purpose of this study is to learn more about the health of female children with congenital adrenal hyperplasia (CAH) and how CAH affects the child's life from the perspective of the child and caregiver. The study consists questions which should take about 10 minutes or less of your time.

There is a minimal risk to answering these questions. Some questions may make you feel uncomfortable, but they are important questions that help us understand how CAH affects your child's life. There is the potential risk of loss of confidentiality. However, every effort will be made to keep your information confidential.

You will not receive any direct benefits from being in this study. The results of this study may help people with $\mathrm{CAH}$ in the future and benefit society by increasing knowledge about the health-related quality of life of children with $\mathrm{CAH}$.

Participation is voluntary. You may stop participating in this study at any time while you are answering questions.

All electronic records containing your information will be computer password protected and accessible only to research personnel, except as required by law (such as reports of child abuse, plans to commit suicide, etc.).

You will receive a one-time $\$ 20$ gift card to a retail store when you finish answering questions. If you are 12 years and older, the gift card will be given to you; however, if you are under 12 years old, the gift card will be given to your parents for your use.

Contact Lacretia Carroll at 901-232-1682 or 1carrol6@uthsc.edu if you have questions about your participation in this study, or if you have questions, concerns, or complaints about the research.

Do you knowingly and freely choose to participate in the study?

1. Yes

2. No 


\section{VITA}

Lacretia Carroll, born in 1989, received a Bachelor's of Science in Nursing degree from the University of Alabama Huntsville in 2011. After gaining experience as a staff nurse in the Orthopedic and Neurosurgical units, she obtained a Master of Science in Nursing degree focusing on Nursing Informatics from the University of Memphis in 2013. Her 6.5 years of nursing experience includes work in the areas of med-surg nursing, pediatric neurosurgical nursing, pediatric intensive care, and clinical research within the areas of Endocrinology, Asthma and Immunology.

While a doctoral student at the University of Tennessee Health Science Center, Lacretia served on the Graduate Student Executive Committee as the Community Outreach Representative and Nursing PhD Representative as well as serving on the Electronic Thesis and Dissertation Committee. Her research focused on health-related quality of life and female children with congenital adrenal hyperplasia and was funded by the Beta Theta-at-Large chapter of Sigma Theta Tau International Honor Society of Nursing, the University of Tennessee Health Science Center College of Nursing, and the Cares Foundation of New York. She has presented her research at local, regional, and national meetings including Pediatric Research Day, Society of Pediatric Nursing Annual Conference, Southern Nursing Research Society, and the International Congress of Qualitative Inquiry. Lacretia also authored and published a book titled, The CAH Wonderclub which is available in print and online.

Lacretia will receive her Doctor of Philosophy degree in Nursing Science in May 2018. She is currently a clinical research coordinator at Le Bonheur Children's Hospital in Memphis, TN. 